University of Tennessee Health Science Center

UTHSC Digital Commons

$12-2016$

\title{
Dissecting the Physiological Roles of ULK1/2 in the Mouse Brain
}

Bo Wang

University of Tennessee Health Science Center

Follow this and additional works at: https://dc.uthsc.edu/dissertations

Part of the Medical Physiology Commons, and the Neurosciences Commons

\section{Recommended Citation}

Wang, Bo (http://orcid.org/0000-0001-6898-9757), "Dissecting the Physiological Roles of ULK1/2 in the Mouse Brain" (2016). Theses and Dissertations (ETD). Paper 415. http://dx.doi.org/10.21007/ etd.cghs.2016.0419.

This Dissertation is brought to you for free and open access by the College of Graduate Health Sciences at UTHSC Digital Commons. It has been accepted for inclusion in Theses and Dissertations (ETD) by an authorized administrator of UTHSC Digital Commons. For more information, please contact jwelch30@uthsc.edu. 


\title{
Dissecting the Physiological Roles of ULK1/2 in the Mouse Brain
}

\begin{abstract}
Mammalian UNC-51-like kinases 1 and 2 (ULK1 and ULK2), Caenorhabditis elegans UNC-51 and Drosophila melanogaster Atg1 are redundant serine/threonine kinases that regulate flux through the autophagy pathway in response to various types of cellular stress. C. elegans UNC-51 and D. melanogaster Atg1 also promote axonal growth and defasciculation, and disruption of these genes results in defects in axon guidance in invertebrates. Germline Ulk1/2-deficient mice die perinatally. Therefore, we used a conditional-knockout approach to investigate the roles of ULK1/2 in the brain. Mice lacking Ulk1 and Ulk2 in their central nervous systems (CNS) showed defects in axonal pathfinding and defasciculation affecting the corpus callosum (CC), anterior commissure (AC), corticothalamic axons (CTAs) and thalamocortical axons (TCAs) and mossy fibers. These defects led to impaired midline crossing of callosal axons, anterior commissure hypoplasia and disorganization of the somatosensory cortex. The axon guidance defects observed in Ulk1/2 double knockout (dko) and in CNS-specific (NestinCre) Ulk1/2 conditional double knockout (cdko) mice were not recapitulated in mice lacking other autophagy genes (i.e. Atg7 or Fip200), and was associated with abnormal localization of the axon guidance molecule, transient axonal glycoprotein-1 (TAG-1) in the distal CTAs. Approximately $40 \%$ of the Ulk $1 / 2$ cdko animals died shortly after birth; the remaining animals survived up to 4 months. Although the mice showed neuronal degeneration, specifically in the hippocampal CA1 region, the neurons showed no accumulation of $\mathrm{P} 62+$ /ubiquitin+ inclusions or abnormal membranous structures, which are observed in mice lacking other autophagy genes, such as Atg7, and Fip200. Rather, neuronal death was associated with activation of the unfolded protein response (UPR) pathway. An unbiased proteomics approach identified SEC16A as a novel ULK1/2-interacting partner. ULK-mediated phosphorylation of SEC16A regulated the assembly of endoplasmic reticulum (ER) exit sites and ER-to-Golgi trafficking of specific cargo such as, the serotonin transporter SERT, and did not require other autophagy proteins (e.g. ATG13). The defect in ER-to-Golgi trafficking activated the UPR pathway in ULK-deficient cells; both processes were reversed upon expression of SEC16A with a phosphomimetic substitution. Thus, the regulation of ER-to-Golgi trafficking by ULK1/2 is essential for cellular homeostasis. Moreover, the defect in SERT trafficking may also contribute to the disrupted formation of the barrel cortex in the Ulk $1 / 2$ cdko mice. Together, these data highlight the autophagy-independent role of ULK1 and ULK2 in maintaining cellular homeostasis and regulating axon guidance in the mammalian brain.
\end{abstract}

Document Type

Dissertation

Degree Name

Doctor of Philosophy (PhD)

Program

Biomedical Sciences

Research Advisor

Mondira Kundu, MD, PhD

Keywords

Axon guidance, ER stress, ER-to-Golgi trafficking, FIP200, SEC16A, ULK/ATG1

\section{Subject Categories}

Medical Physiology | Medical Sciences | Medicine and Health Sciences | Neurosciences 
Comments

One year embargo expires November 2017.

This dissertation is available at UTHSC Digital Commons: https://dc.uthsc.edu/dissertations/415 
Dissecting the Physiological Roles of ULK1/2 in the Mouse Brain

\author{
A Dissertation \\ Presented for \\ The Graduate Studies Council \\ The University of Tennessee \\ Health Science Center \\ In Partial Fulfillment \\ Of the Requirements for the Degree \\ Doctor of Philosophy \\ From The University of Tennessee
}

By

Bo Wang

December 2016 
Chapter 2, Chapter 3, and portions of Chapter 5 (C) 2016 by Elsevier. All other material C 2016 by Bo Wang. All rights reserved. 


\section{ACKNOWLEDGEMENTS}

The past 5 years has been an amazing adventure. As an international student, this experience of working towards doctoral degree in a foreign country is unforgettable and priceless. In retrospect, I am full of appreciation to those who have facilitated my study in the United States. My greatest and most sincere thanks go to my mentor, Dr. Mondira Kundu. She guided me through the past four and a half years, and built me in various different ways. As a mentor, she is extremely patient, encouraging, supportive, and instrumental. And because of that, I had the chance to explore many scientific issues that interested me. A student really couldn't ask for more from a mentor. More importantly, her passion and meticulousness in science inspired me tremendously to pursue a career in academia in the past and in the future. Although I will soon leave her laboratory, the skills that I learned here will always stay with me, and for that, she deserves every single credit.

I have also had the privilege of working with all the colleagues in the lab. They are extremely patient with me and literally helped me a million times. Often, they prioritized my needs and carried me through hard times, without which I couldn't fulfill my goals here. They are happy for my achievement and sorry for my loss, making me feel at least I am not alone here in the United States. We share countless memories and moments, and I will carry those wherever I will be in the future. You will be deeply missed.

I want to thank all the people along the ways. My committee members including: Dr. Kristin M. Hamre, Dr. Joseph T. Opferman, Dr. David J. Solecki, and Dr. Paul J. Taylor have provided significant insight into my research. They devoted their time and effort generously and guided me through all the years until now. Their guidance will be my lifelong treasure.

During the study, I have been very lucky to receive tremendous assistance from fantastic collaborators and the shared resources and support by St. Jude Children's Research Hospital. Especially, I would like to thank Dr. Xinwei Cao, Dr. Linda M. Hendershot, and Dr. Douglas R. Green for offering critical reagents and useful comments for my projects. The Veterinary Pathology Core, Cell and Tissue Imaging Core run by Dr. Peter A. Vogel, Dr. Victoria C. Frohlich, and Mrs. Sharon Frase, respectively, have provided enormous excellent technical support for my studies.

Last but not least, I am grateful that I had the chance to work at St. Jude Children's Research Hospital, where everyone works together to cure pediatric cancer. The unprecedented dedication and enthusiasm of everyone here has given me constant encouragement. As a researcher in basic biomedical sciences, treating human diseases remains my ultimate goal. I hope my efforts during the past few years has contributed towards that goal. 


\begin{abstract}
Mammalian UNC-51-like kinases 1 and 2 (ULK1 and ULK2), Caenorhabditis elegans UNC-51 and Drosophila melanogaster Atg1 are redundant serine/threonine kinases that regulate flux through the autophagy pathway in response to various types of cellular stress. C. elegans UNC-51 and D. melanogaster Atg1 also promote axonal growth and defasciculation, and disruption of these genes results in defects in axon guidance in invertebrates. Germline $U l k 1 / 2$-deficient mice die perinatally. Therefore, we used a conditional-knockout approach to investigate the roles of ULK1/2 in the brain. Mice lacking $U l k 1$ and $U l k 2$ in their central nervous systems (CNS) showed defects in axonal pathfinding and defasciculation affecting the corpus callosum (CC), anterior commissure (AC), corticothalamic axons (CTAs) and thalamocortical axons (TCAs) and mossy fibers. These defects led to impaired midline crossing of callosal axons, anterior commissure hypoplasia and disorganization of the somatosensory cortex. The axon guidance defects observed in Ulk1/2 double knockout (dko) and in CNS-specific (NestinCre) $U l k 1 / 2$ conditional double knockout (cdko) mice were not recapitulated in mice lacking other autophagy genes (i.e. Atg7 or Fip200), and was associated with abnormal localization of the axon guidance molecule, transient axonal glycoprotein-1 (TAG-1) in the distal CTAs. Approximately $40 \%$ of the $U l k 1 / 2$ cdko animals died shortly after birth; the remaining animals survived up to 4 months. Although the mice showed neuronal degeneration, specifically in the hippocampal CA1 region, the neurons showed no accumulation of $\mathrm{P}^{+} 2^{+} / \mathrm{ubiquitin}{ }^{+}$inclusions or abnormal membranous structures, which are observed in mice lacking other autophagy genes, such as Atg7, and Fip200. Rather, neuronal death was associated with activation of the unfolded protein response (UPR) pathway. An unbiased proteomics approach identified SEC16A as a novel ULK1/2interacting partner. ULK-mediated phosphorylation of SEC16A regulated the assembly of endoplasmic reticulum (ER) exit sites and ER-to-Golgi trafficking of specific cargo such as, the serotonin transporter SERT, and did not require other autophagy proteins (e.g. ATG13). The defect in ER-to-Golgi trafficking activated the UPR pathway in ULKdeficient cells; both processes were reversed upon expression of SEC16A with a phosphomimetic substitution. Thus, the regulation of ER-to-Golgi trafficking by ULK1/2 is essential for cellular homeostasis. Moreover, the defect in SERT trafficking may also contribute to the disrupted formation of the barrel cortex in the Ulk1/2 cdko mice. Together, these data highlight the autophagy-independent role of ULK1 and ULK2 in maintaining cellular homeostasis and regulating axon guidance in the mammalian brain.
\end{abstract}




\section{TABLE OF CONTENTS}

CHAPTER 1. INTRODUCTION ...............................................................................

ULK/ATG1 Regulates the Cellular Response to Metabolic Stress ...............................1

The Canonical Autophagy-Inducing ULK/ATG1 Kinase Complex ..........................

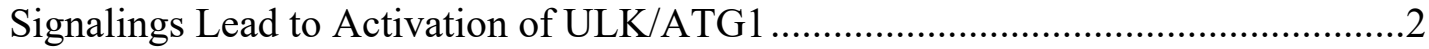

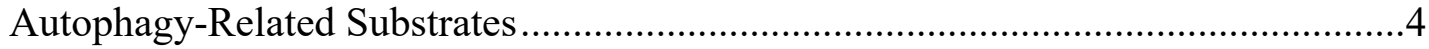

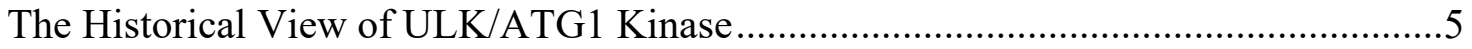

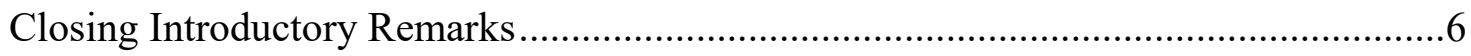

CHAPTER 2. EXPERIMENTAL PROCEDURES...................................................9

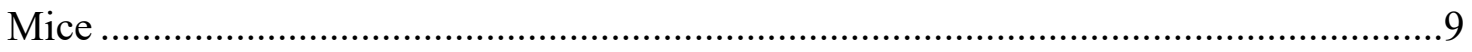

Immunostainings and Histologic Analyses....................................................

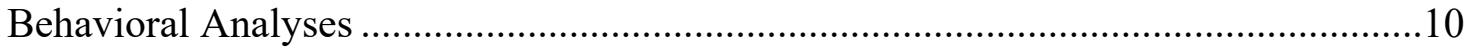

Morphometric Analyses of Electron Micrographs ................................................... 10

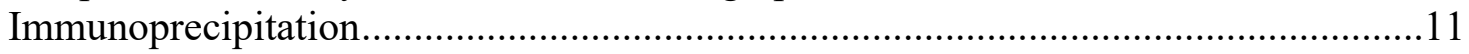

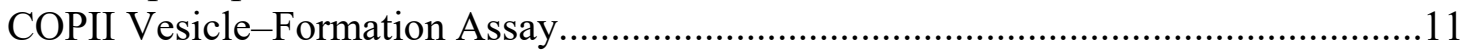

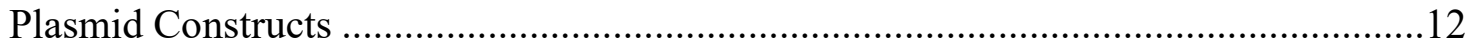

Generation of Murine Embryonic Fibroblasts, Cell Culture, Transfection, Gene

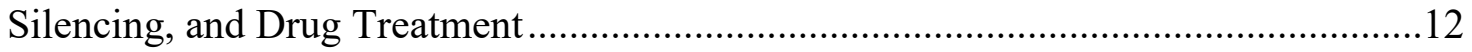

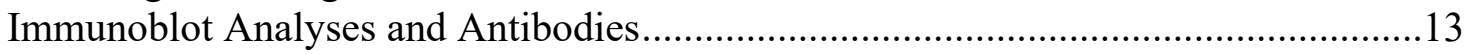

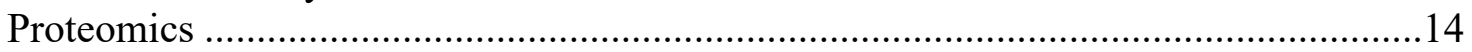

Quantitative Real-Time PCR .............................................................................. 14

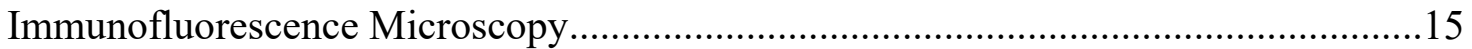

Immunofluorescence Microscopy in C. elegans .....................................................15

Endoglycosidase H Assay................................................................................ 16

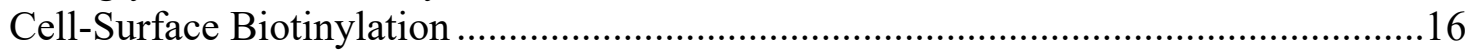

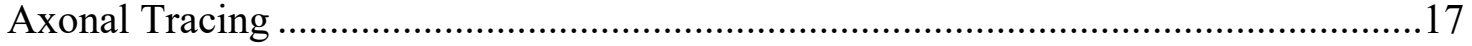

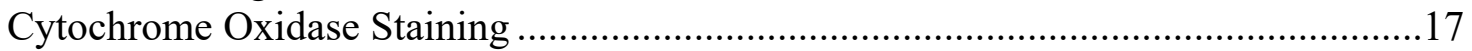

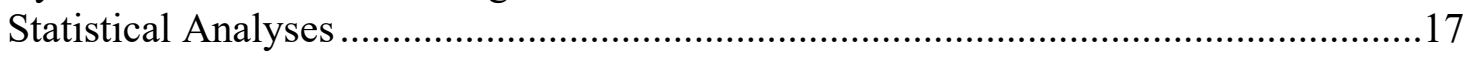

CHAPTER 3. THE NONCANONICAL ROLE OF ULK/ATG1 IN ER-TOGOLGI TRAFFICKING IS ESSENTIAL FOR CELLULAR HOMEOSTASIS .....18

Mice Lacking Ulk1/Ulk2 Expression in the CNS Have a Distinct Pattern of

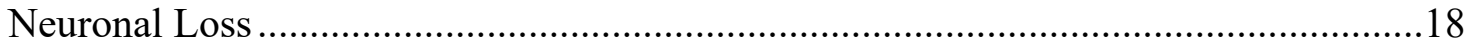

ULK1/2 Deficiency Activates the Unfolded Protein Response in Hippocampal

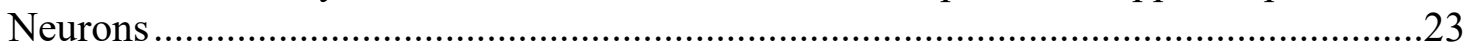

ULK1 and ULK2 Mediate the Phosphorylation of SEC16A ...................................26

ULK1 Activity Regulates the Budding of COPII Vesicles in Vitro .............................29

ULK1 and Related Kinases Have a Conserved Role in Assembling COPII

Components at ERES............................................................................................29

ULK1 and Related Kinases Regulate ER-to-Golgi Trafficking .................................32

ULK-Mediated Phosphorylation of SEC16A at S846 Is Required for ER-to-Golgi

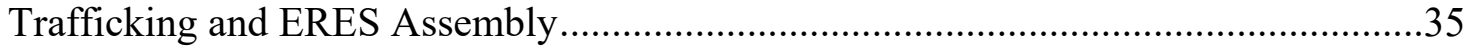


The Defect in ER-to-Golgi Trafficking in ULK-Deficient Cells Leads to an ER-

Stress Response That Is Reversed by the S846D SEC16A Mutant.

\section{CHAPTER 4. AUTOPHAGY-INDEPENDENT ROLE OF ULK1 AND ULK2 IS ESSENTIAL FOR AXON GUIDANCE IN THE DEVELOPING MOUSE} FOREBRAIN

Ulk1/2-Deficiency in the CNS Results in Abnormal Axon Guidance.

ULK1 and ULK2 Regulate Axon Guidance via an Autophagy-Independent

Pathway..... .43

ULK1 and ULK2 Are Not Essential for Cortical Neurogenesis .47

ULK1 and ULK2 Regulate the Midline Crossing of Callosal Axons .47

Ulk1/2-Deficiency Impairs Organization of the Somatosensory Cortex

TAG-1 Is Mislocalized in Corticothalamic Axons

CHAPTER 5. DISCUSSION .......................................................................................56

The Role of ULK/ATG1 in ER-to-Golgi Trafficking Is Essential for Cellular

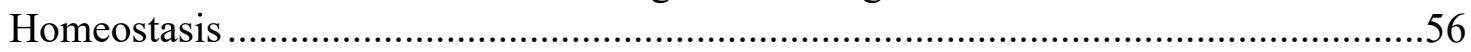

ULK1/2 Are Essential for Axon Guidance in the Developing Mouse Forebrain...........57

Emerging Noncanonical Functions of ULK/ATG1 ....................................................59

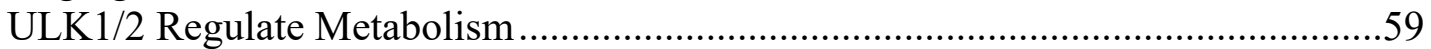

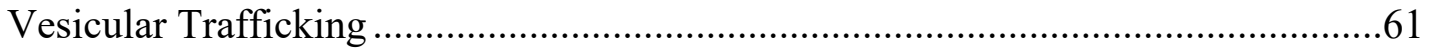

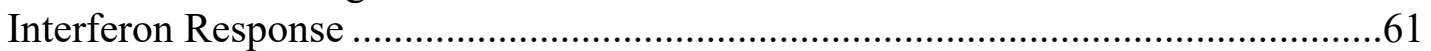

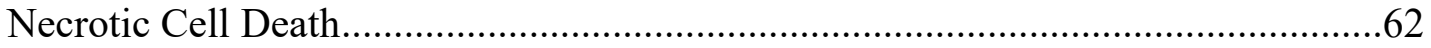

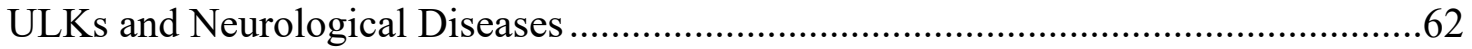

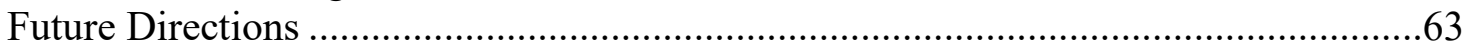

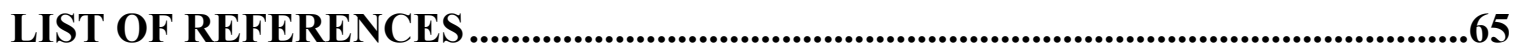

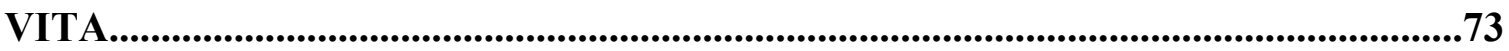




\section{LIST OF FIGURES}

Figure 1-1. ULK/ATG1 drives cellular responses to metabolic stresses............................3

Figure 1-2. The historical view of ULK/ATG1 kinase.....................................................

Figure 3-1. Generation and behavioral characterization of Ulk1/2 cdko mice................19

Figure 3-2. Degeneration of pyramidal neurons in the CA1 region of ULK1/2deficient mice.

Figure 3-3. Ulk1/2 deficiency in hippocampal neurons is associated with activation of the UPR pathway.

Figure 3-4. ULKs mediate the phosphorylation of SEC16A and facilitate in vitro budding of COPII vesicles.

Figure 3-5. ULKs regulate the assembly of COPII complexes at ERES..........................30

Figure 3-6. ULKs regulate ER-to-Golgi trafficking......................................................33

Figure 3-7. ULK-mediated phosphorylation of SEC16A at S846 is required for assembly of SEC24C $\mathrm{C}^{+}$ERES and ER-to-Golgi trafficking.....

Figure 3-8. SEC16A mutant with the phosphomimetic S846D overcomes the defect in ER-to-Golgi trafficking and prevents activation of the UPR in ULKdeficient cells.

Figure 4-1. ULK1 and ULK2 are required for axonal guidance in the forebrain.

Figure 4-2. ULK1 and ULK2 are not required for autophagy-mediated turnover of ubiquitinated protein in the cerebral cortex.

Figure 4-3. FIP200 and ATG7 are not required for axon guidance in the forebrain.......45

Figure 4-4. ULK1 and ULK2 regulate mossy fiber guidance via an autophagy independent-mechanism.

Figure 4-5. ULK1 and ULK2 are not essential for neurogenesis.

Figure 4-6. ULK1 and ULK2 regulate development of the corpus callosum.

Figure 4-7. ULK1 and ULK2 are required for proper organization of the somatosensory cortex.

Figure 4-8. Abnormal axonal fasciculation in the Ulk1/2-deficient animals is associated with mislocalization of TAG-1 in the projection neurons. 
Figure 5-1. The versatile functions of ULK/ATG1 beyond autophagy regulation. ........60 


\section{LIST OF ABBREVIATIONS}

$\mathrm{AC}$

AMPK

ATF6

ATG5

ATG7

ATG13

ATG14

$\mathrm{BiP}$

BP

CA1

C. elegans

$\mathrm{CC}$

cdko

cGAMP

cGAS

CHOP

CNS

$\mathrm{CO}$

COPII

CTA

Cvt pathway

$\mathrm{DH}$
Anterior commissure

5' AMP-activated protein kinase

Activating Transcription Factor 6

Autophagy related 5

Autophagy related 7

Autophagy Related 13

Autophagy Related 14

Binding immunoglobulin protein

Blocking peptide

Region I of hippocampus proper

Caenorhabditis elegans

Corpus callosum

Conditional double knockout

Cyclic guanosine monophosphate-adenosine monophosphate

cGAMP synthase

DNA damage-inducible transcript 3

Central nervous system

Cytochrome oxidase

Coat protein complex II

Corticothalamic axon

Cytoplasm-to-vacuole targeting

Dendate hilus 


\begin{tabular}{|c|c|}
\hline DiI & $\begin{array}{l}1,1^{\prime} \text {-dioctadecyl-3,3,38,38, tetramethylindocarbocyanine } \\
\text { perchlorate }\end{array}$ \\
\hline D. melanogaster & Drosophila melanogaster \\
\hline $\mathrm{dTh}$ & Dorsal thalamus \\
\hline $\mathrm{EC}$ & External capsule \\
\hline $\mathrm{eIF} 2 \alpha$ & Eukaryotic Initiation Factor $2 \alpha$ \\
\hline Endo $\mathrm{H}$ & Endoglycosidase $\mathrm{H}$ \\
\hline ER & Endoplasmic reticulum \\
\hline ERES & ER exit sites \\
\hline FIP200 & RB1-inducible coiled-coil protein 1 \\
\hline GFAP & Glial fibrillary acidic protein \\
\hline $\mathrm{HC}$ & Hippocampal commissure \\
\hline $\mathrm{H} \& \mathrm{E}$ & Hematoxylin and eosin \\
\hline IBA1 & Ionized calcium-binding adapter molecule 1 \\
\hline IC & Internal capsule \\
\hline IFN & Interferon \\
\hline $\mathrm{ipb}$ & Infrapyramidal bundle of mossy fibers \\
\hline IPs & Immunoprecipitates \\
\hline IRF3 & Interferon regulatory factor 3 \\
\hline ISGs & Interferon stimulated genes \\
\hline KI & Kinase inactive \\
\hline L1 & Neural adhesion molecule L1 \\
\hline MAPK & Mitogen-activated protein kinases \\
\hline MEFs & Murine embryonic fibroblasts \\
\hline
\end{tabular}




\begin{tabular}{|c|c|}
\hline mTOR & Mechanistic target of rapamycin \\
\hline NCAM & Neural cell adhesion molecule \\
\hline NeuN & Neuronal nuclei \\
\hline NF165 & Neurofilament $165 \mathrm{kDa}$ \\
\hline NHS & N-hydroxysuccinimido \\
\hline NSM & Neurosecretory motor \\
\hline PAS & Preautophagosomal structure \\
\hline P62/SQSTM1 & Sequestosome 1 \\
\hline $\mathrm{Pb}$ & Probst bundle \\
\hline PI3K & Phosphatidylinositol 3-kinase \\
\hline PM & plasma membrane \\
\hline PNGase & Peptide-N-glycosidase F \\
\hline PARP1 & Poly (ADP-ribose) polymerase 1 \\
\hline polyQ-Htt & Polyglutamine expanded huntingtin \\
\hline PSPB & Pallial-subpallial boundary \\
\hline Ser & Seronine \\
\hline SERT & Serotonin transporter \\
\hline SO & Stratum oriens; \\
\hline $\mathrm{Spb}$ & Suprapyramidal bundle of mossy fibers \\
\hline SR & Stratum radiatum \\
\hline STING & Stimulator of interferon genes \\
\hline TAG-1 & Transient axonal glycoprotein-1 \\
\hline Tbr1 & T-Box, Brain 1 \\
\hline
\end{tabular}


TCA Thalamocortical axon

ULK1 UNC-51-like kinase 1

ULK2 UNC-51-like kinase 2

UPR Unfolded protein response

VPL Ventral posterolateral nucleus

VPM Ventral posteromedial nucleus

VSV-G Vesicular stomatitis virus (VSV) G-protein

WGA Wheat germ agglutinin

XBP1 X-Box Binding Protein 1

5-HT Serotonin 


\section{CHAPTER 1. INTRODUCTION}

Autophagy is a catabolic process that occurs under basal physiological and stressed conditions. Cargoes, including superfluous organelles or proteins, are enclosed in double membrane-bound autophagosomes and degraded in lysosomes (Levine and Kroemer, 2008; Mizushima et al., 2008). As an evolutionarily conserved mechanism, autophagy is required for cellular homeostasis from yeast to mammals (Levine and Kroemer, 2008; Mizushima et al., 2008). It is widely accepted that autophagy dysregulation contributes to a variety of human diseases, including neurodegeneration, cancer, and infectious diseases (Levine and Kroemer, 2008; Mizushima et al., 2008).

To date, more than 30 autophagy-related $(A T G)$ genes have been identified in yeast - the majority of which have homologues or counterparts in mammals (Feng et al., 2014). $A T G$ genes can be grouped into several functional units: the ULK/ATG1 complex, which activates the VPS34/PI3K complex and promotes AT9-mediated membrane recycling; the VPS34/PI3K complex, which is essential for membrane nucleation; the two ubiquitin-like conjugation systems (Atg12-Atg5 and Atg8/LC3); Atg2-Atg18 complex; and the transmembrane protein Atg9 (Feng et al., 2014).

Since ULK/Atg1 is the only kinase in the autophagy pathway, and kinases are well accepted druggable targets for therapeutic purposes, ULK/ATG1 has gained much attention in the past decade. While potent inhibitors of ULK kinases have been developed to target autophagy in the context of diseases (Egan et al., 2015; Petherick et al., 2015), how ULK/ATG1 drives the cellular responses to physiological vs. stressed conditions is not clear. A better understanding about the function of ULK/Atg1 will help to improve the specificity of the inhibitors and minimize potential side effects in therapeutic outcomes.

\section{ULK/ATG1 Regulates the Cellular Response to Metabolic Stress}

\section{The Canonical Autophagy-Inducing ULK/ATG1 Kinase Complex}

ULK/ATG1 is the only known kinase with a specific role in autophagy. In yeast, disruption of Atg1 function abrogates autophagy at an early stage, leading to the conclusion that Atg1 functions at the initial step of autophagy (Matsuura et al., 1997). Meanwhile, yeast also utilizes the cytoplasm-to-vacuole targeting ( $\mathrm{Cvt}$ ) pathway to transport two vacuolar enzymes, aminopeptidase I and a-mannosidase, to the vacuole-a process that is dependent on Atg1 (Harding et al., 1995). Atg1 is reported to interact with at least eight other autophagy genes, some of which are only required for autophagy regulation (Atg13, Atg17, Atg29, Atg31), while the others (Atg11, Atg20, Atg24 and Vac8) are essential specifically for the Cvt pathway (Mizushima, 2010). As Cvt proceeds constitutively under nutrient-rich conditions and the Atg1-mediated autophagy occurs after nutrient depletion, it is thought that Atg1 may function as a switch between 
autophagy and the Cvt pathway through post-translational modifications or shifting its associations with other interacting partners (Mizushima, 2010). Atg1 does not interact with Atg13 or Atg17 under nutrient-rich conditions but can bind to Atg13 upon starvation, and the increased binding of Atg13 upregulates its kinase activity (Kamada et al., 2000). The mammalian homolog of Atg1, ULK1, was first shown to regulate autophagy in a kinase screening for novel regulators of autophagy in response to amino acid starvation. ULK2, a close homologue of ULK1, plays a partially redundant role in regulating autophagy (Cheong et al., 2011; Lee and Tournier, 2011). The mammalian ULK complex consists of ULK1 and ULK2, ATG13, FIP200 (the mammalian counterpart of yeast Atg17), and ATG101. ATG13 and FIP200 modulate proper localization, stability, and kinase activity of ULK1 and ULK2 (Ganley et al., 2009; Hosokawa et al., 2009; Jung et al., 2009). Nevertheless, unlike the yeast Atg1-Atg13Atg17, the ULK complex exists constitutively, irrespective of nutritional conditions (Ganley et al., 2009; Hosokawa et al., 2009; Jung et al., 2009), suggesting differential regulation of autophagy in different organisms. In addition, there appears to be no equivalent of the yeast Cvt pathway in mammalian cells. It remains to be seen whether the mechanism by which Atg1 delivers vacuole enzymes is inherited by mammals during evolution.

\section{Signalings Lead to Activation of ULK/ATG1}

Much effort has been devoted to the search for novel ULK/ATG1 substrates and towards understanding how the ULK/ATG1 complex is regulated by upstream regulators in response to cellular nutrient status (Figure 1-1). Under fed conditions, yeast Atg13 is hyperphosphorylated by TOR (target of rapamycin) kinase but is rapidly dephosphorylated when TOR is inhibited by starvation (Kamada et al., 2000; Kamada et al., 2010). As a consequence, it promotes Atg1-Atg13-Atg17 complex formation and the kinase activity of Atg1 (Kamada et al., 2000; Kamada et al., 2010). It is not yet clear if TOR also phosphorylates Atg1 under basal condition (Papinski and Kraft, 2016). In a slightly different fashion, both mammalian ULK1 and ATG13 are phosphorylated by mTOR under normal conditions, and they are quickly de-phosphorylated upon starvation, thus activating the ULK complex (Alers et al., 2012; Ganley et al., 2009; Hosokawa et al., 2009; Jung et al., 2009; Kim et al., 2011). Meanwhile, two studies almost simultaneously demonstrated that following glucose or Earle's Balanced Salt Solution (EBSS) starvation, activated AMPK directly phosphorylates ULK1 at Ser467, Ser555, Thr574, and Ser637 (Egan et al., 2011; Kim et al., 2011). Although phosphorylation of ULK1 by AMPK has no impact on the kinase activity of ULK1, it is sufficient to modulate the function of ULK complex (Alers et al., 2012; Egan et al., 2011; Kim et al., 2011; Mack et al., 2012). Thus, mTOR and AMPK work mutually to sense the metabolic cues and relay the signals to ULK complex. Independent of AMPK, ULK1 can be activated by the GSK3-TIP60 signaling axis upon growth factor deprivation. It is shown that depletion of growth factors from the cells triggers deinhibition of GSK3, which then directly phosphorylates the acetyltransferase TIP60 at Ser86. The activated TIP60 subsequently acetylates ULK1 to induce autophagy (Lin et al., 2012). Although cAMPdependent protein kinase A (PKA) has been reported to phosphorylate yeast Atg1 


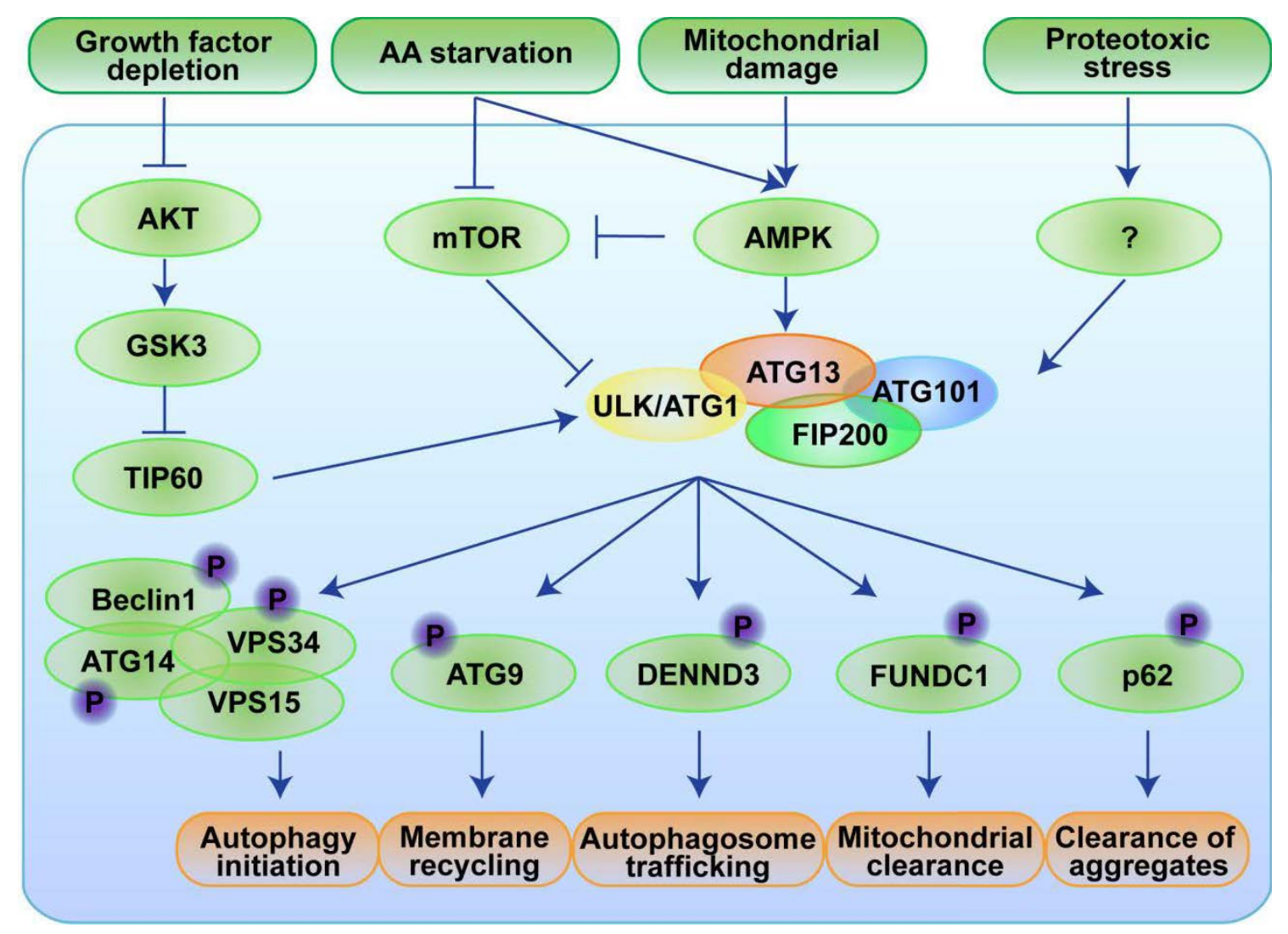

Figure 1-1. ULK/ATG1 drives cellular responses to metabolic stresses.

Multiple nutrient-sensing pathways such as AKT-GSK3 axis, mTOR-AMPK axis, and some other unknown molecules for extracellular or intracellular stimuli impinge on ULK/ATG1 to achieve different regulatory effects mainly through posttranslational modification. The inhibited AKT, due to growth factor depletion, leads to activation of GSK3 and subsequent activation of TIP60, which catalyzes acetylation of ULK1 to induce autophagy. ULK/ATG1 is normally inhibited by mTOR. The energy sensor AMPK can release the suppressive effects of mTOR on ULK complex to promote autophagy. Some unknown molecules may exist to detect intracellular protein aggregates and signal the proteomic stress to the ULK complex to enhance protein degradation. Once activated by upstream regulators, ULK/ATG1 phosphorylates different substrates to regulate autophagy. The established substrates of ULK/ATG1 include Beclin1, VPS34, and ATG14 of the VPS34 complex as well as Atg9, DENND3, FUNDC1, and P62. The phosphorylated substrates of ULK/ATG1 kinase have been implicated to function at different steps of autophagy, such as initiation, membrane recycling, and autophagosome trafficking, etc. See text for details. 
(Budovskaya et al., 2005), the potential link between mammalian PKA and ULK1/2 has yet to be established.

\section{Autophagy-Related Substrates}

The autophagy-related substrates of ULK/ATG1 can be categorized into at least four different groups: (i) ULK complex, comprising of ULK1, ATG13, FIP200, and ATG101, (ii) VPS34 complex, including Beclin-1, VPS34, and ATG14, (iii) ATG9, and, (iv) other substrates that are selective for certain conditions. While mammalian ULK1 was found to autophosphorylate Thr180, located in the kinase activation loop essential for intact kinase activity of ULK1 (Bach et al., 2011), it has been reported to phosphorylate ATG13 at Ser318, a modification required for targeting ATG13 to depolarized mitochondria (Joo et al., 2011). There is some evidence that ULK1 phosphorylates human FIP200 at Ser934, Ser986, and Ser1323, though the functional significance of these phosphorylation events has not yet been determined (Egan et al., 2015). Ser11 and Ser203 of human ATG101 are reported to be phosphorylated in an ULK1-dependent manner (Egan et al., 2015). Similar to the case of FIP200, how the phosphorylation of ATG101 contributes to autophagy is not clear. It is noteworthy that not all the reported phosphorylation sites on the putative substrates of ULKs are conserved, indicating that ULK complex may be regulated in a species-specific manner. Independent laboratories have identified Beclin-1, VPS34, and ATG14 (components of the VPS34 complex) as bona fide substrates of ULK1 during amino acid (AA) starvation (Egan et al., 2015; Park et al., 2016; Russell et al., 2013). By phosphorylating human Beclin-1, VPS34, and ATG14 at Ser14, Ser249, and Ser29, respectively, ULK1 enhances the activity of VPS34 complex to promote autophagosome formation (Egan et al., 2015; Park et al., 2016; Russell et al., 2013). ATG1/ULK has been implicated in Atg9/ATG9 cycling in both yeast and mammalian cells. In yeast, Atg9 cycles between the preautophagosomal structure (PAS) and the peripheral sites, in an Atg1-dependent manner (Reggiori et al., 2004). Recent studies, however, have demonstrated that Atg1mediated phosphorylation of Atg9 at multiple sites is required for recruitment of Atg8 and Atg18 to the PAS (Papinski et al., 2014). Nevertheless, phosphorylation of Atg9 by Atg1 is not necessary for its shuttling between PAS and cytosol, as phosphor-mutant Atg9 localizes to the PAS properly, in contrast to the abnormal accumulation of Atg9 in the PAS in yeast with Atg1 deletion, suggesting that Atg1 modulates Atg9 function by both kinase-dependent and -independent mechanisms (Papinski et al., 2014). Whether ULK1(2) governs ATG9 cycling in the mammals is not as clear. An earlier reports showed that knockdown of ULK1 abolished starvation-induced redistribution of ATG9 (Young et al., 2006), recent work suggests that independent of ULK1, ATG9 can be recruited to DFCP1-positive phagophores (Orsi et al., 2012). It is yet to be determined whether mammalian ATG9 is also a substrate of ULK1(2) and whether the mechanisms how Atg1 governs the Atg9 function are conserved.

In addition to targeting various components of the autophagy machinery, several proteins not typically regarded as autophagy molecules are also bona fide substrates of ULK/ATG1 under certain conditions. FUNDC1 is a mitochondrial outer-membrane 
protein that acts as a cargo receptor protein in hypoxia-induced mitophagy.

Phosphorylation of mouse FUNDC1 at Ser17 by ULK1 is required for efficient binding of LC3 to FUNDC1 and mitophagy progression (Wu et al., 2014). Following EBSS treatment, the guanine nucleotide exchange factor DENND3 is phosphorylated at Ser554 and Ser572 in an ULK1 - and ULK2-dependent manner (Xu et al., 2015). The phosphorylation promotes activation of Rab12, a small GTPase that regulates autophagosome trafficking. The idea that ULK1 and ULK2 regulate autophagosome trafficking is intriguing. The increasing number of substrates of ULK/ATG1 that appear to work at different stages of the autophagy pathway strongly argues that ULK1 and ULK2 function at multiple steps during autophagy induction. However, to verify this concept can be technically difficult, unless distinct mutants of ULK/ATG1 can be created to precisely dissect the upstream and downstream functions of these enzymes.

Alternatively, according to different metabolic cues, ULK/ATG1 can probably target different substrates to achieve differential responses. In fact, a recent study has demonstrated that in response to proteasome inhibition or expression of polyglutamineexpanded huntingtin (polyQ-Htt), ULK1 phosphorylates the autophagy receptor protein p62 at Ser409 to induce clearance of ubiquitinated protein. More importantly, the ULK1mediated phosphorylation does not occur upon nutrient starvation (Lim et al., 2015). Future studies to systematically analyze phosphorylation of known ULK substrates under various stress conditions will provide significant insights into the regulatory mechanism underlying ULK-mediated autophagy.

\section{The Historical View of ULK/ATG1 Kinase}

Although the yeast Atg1 was the first known autophagy-defective strain initially identified in a global screen for autophagy loss-of-function strains (Tsukada and Ohsumi, 1993), the isolation and validation of the metazoan homologs was a twisted story.

C. elegans UNC-51 kinase was originally cloned as a protein critical for axonal development because disrupting unc-51 expression results in uncoordinated movement of C. elegans due to widespread axonal guidance and fasciculation abnormalities in the motor neurons (Ogura et al., 1994). Aberrant vesicles and membranous structures are oftentimes observed in these neurons (McIntire et al., 1992). Similar to unc-51 mutants, D. melanogaster atgl mutants also exhibit abnormal axonal tracts in the ventral nerve cord, including premature truncation and defective midline crossing of longitudinal tracts at the embryonic stage (Toda et al., 2008). Later analysis also revealed abnormal defasciculation of the larval mushroom body axons in D. melanogaster atg 1 mutants (Mochizuki et al., 2011). Mammalian Ulk1 and Ulk2 were first cloned based on their high degree of homology to the C. elegans unc-51 gene (Yan et al., 1998; Yan et al., 1999). And the neuronal function of ULK1 was subsequently demonstrated by independent studies. Dominant-negative expression of $U l k l$ in mouse cultured cerebellar granule neurons inhibits neurite formation and extension (Tomoda et al., 1999). Meanwhile, knocking down the expression of Ulk1, Ulk2, or both, leads to extensive neurite branching and stalled axonal growth in mouse dorsal root ganglion neurons in culture (Zhou et al., 2007). 
While ULK/ATG1 kinase plays evolutionary conserved role in axonal development, the C. elegans UNC-51 turned out to be a close homolog of yeast Atg1, sharing 52.7\% similarity in their N-terminal kinase domain (Matsuura et al., 1997). The role of yeast Atg1 in autophagy regulation led to the finding that UNC-51 is also essential for proper localization of autophagosomal marker proteins and starvation-induced dauer development, an autophagy-dependent process (Melendez et al., 2003). In addition, as observed in the $S$. cerevisiae and $C$. elegans, the ATG1 has been shown to act in autophagy initiation, downstream of Drosophila TOR (dTOR) (Scott et al., 2007). Of note, overexpression of ATG1 is sufficient to induce autophagy (Scott et al., 2007). However, the function of ULK1 in autophagy regulation wasn't discovered until about a decade ago when Tooze lab performed a siRNA screening for essential genes in amino acid starvation-induced autophagy (Chan et al., 2007). Since then, ULK1 and, to a lesser extent, ULK2 have been substantially studied in the context of autophagy regulation under various conditions (Figure 1-2).

\section{Closing Introductory Remarks}

Because of their diverse functions, ULK1 and ULK2 can be potentially linked to various human diseases including neurodegeneration. However, vast majority of studies of ULK1 and ULK2 are performed in vitro, and the physiological relevance of ULK1 and ULK2 is poorly characterized. On the one hand, one of the primary roles of autophagy is to maintain cellular homeostasis through degrading damaged organelles and protein aggregates. Neurons, as one of the few long-lived cell types, heavily rely on autophagy for survival (Nixon, 2013). Thus, it is not surprising that deleting key autophagy genes such as Atg5, Atg7, and Fip200, in the central nervous system (CNS) causes widespread cell death associated with accumulation of p62 and ubiquitin-positive inclusion bodies, a common pathological feature in a variety of human neurological diseases (Hara et al., 2006; Komatsu et al., 2006; Liang et al., 2010). Whether ULK1 and ULK2 are important for neuronal autophagy under physiological conditions is an important question that remains to be answered. On the other hand, the precise in vivo role of ULKs (in axonal development) has not yet been demonstrated unequivocally. Additionally, it is unclear whether autophagy is required for axonal elongation during mammalian neurodevelopment. Since no neuronal functions have been documented for other members of the ULK complex, including ATG13 and FIP200 (Kaizuka and Mizushima, 2015; Liang et al., 2010), it is likely that ULK1 and ULK2 play specialized roles in neurodevelopment, and this might involve a group of separate, novel binding partners. Although Ulk1 single knockout (KO) mice exhibited defects in reticulocyte maturation, no other overt developmental defects were observed (Kundu et al., 2008). No major phenotype has been reported for Ulk2 single KO mice either (Cheong et al., 2011). This suggests at least a partial functional redundancy is shared between ULK1 and ULK2. In line with that, $U l k 1 / 2$ double knockout (dko) mice die shortly after birth, partially due to defective lung development (Cheong et al., 2014). To answer the core question of what is the physiological role(s) of ULK1 and ULK2 in the mouse brain, we generated and characterized the phenotype of CNS-specific Ulk1 and Ulk2 conditional double knockout 


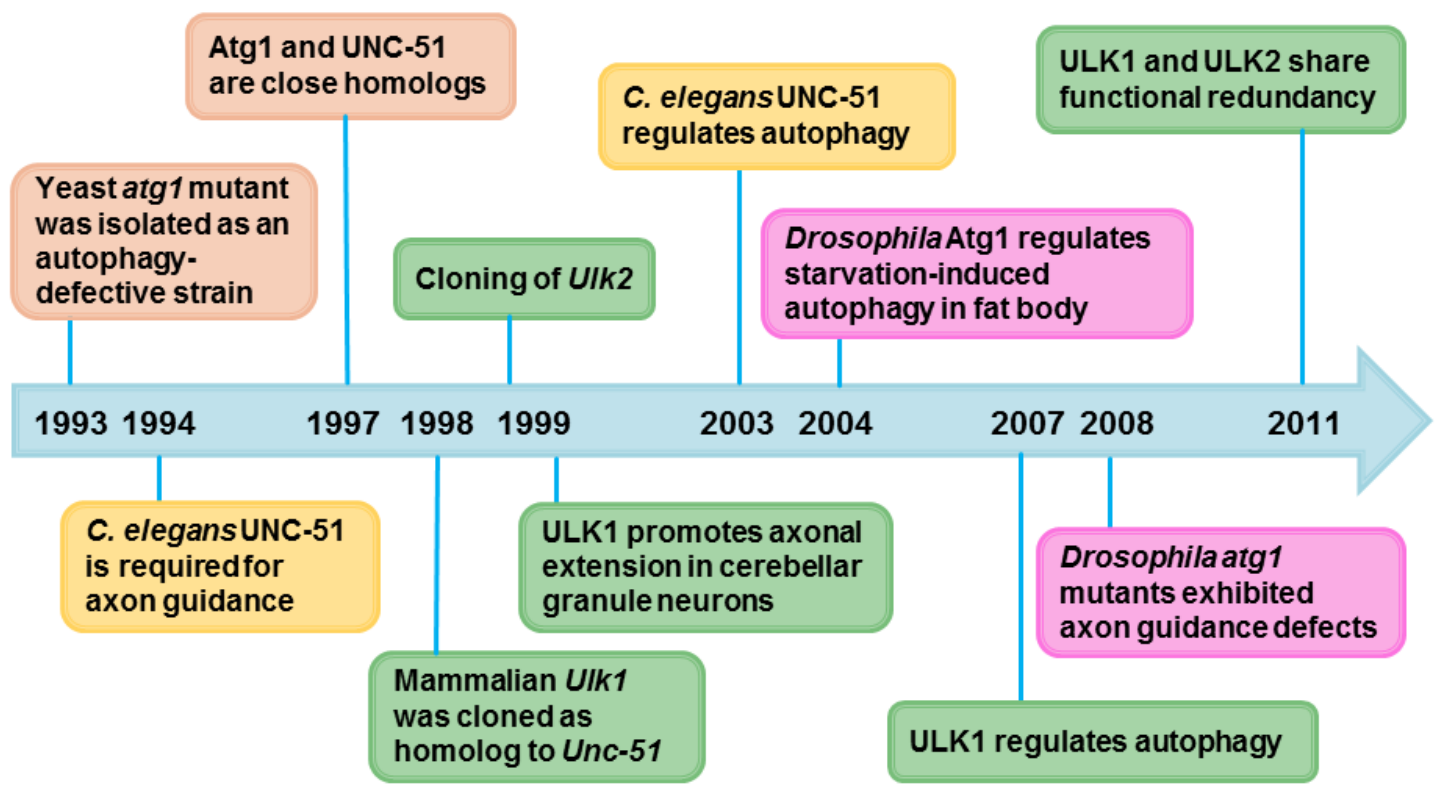

Figure 1-2. The historical view of ULK/ATG1 kinase. 
(Ulk1/2-cdko) mice. The results obtained from the detailed analyses will be described in Chapter 3 and 4. 


\section{CHAPTER 2. EXPERIMENTAL PROCEDURES*}

\section{Mice}

The strategy for targeting the Ulk2 locus was described previously (Cheong et al., 2011). Mice harboring the targeted Ulk2 allele (Ulk $\left.2^{f l o x-n e o}\right)$ were crossed with EIIa-Cre transgenic mice (Jackson Laboratory, stock: 003724), and progeny with Ulk2 ${ }^{k o}$ or Ulk $2^{f l o x}$ alleles were crossed with WT mice to eliminate the expression of the Cre-recombinase. $U l k 1^{-1-}$ mice have been described previously (Kundu et al., 2008). The Nestin-Cre line was obtained from the Jackson Laboratory (stock: 003771). Ulk ${ }^{+/-} ; U l k 2^{\text {floxflox }}$ females were bred with $U l k 1^{+-} ; U l k 2^{--}$;Nestin-Cre males to generate Ulk1/2-cdko (Ulk1 ${ }^{-1} ; U l k 2^{-}$ flox;Nestin-Cre) mice. Atg ${ }^{f f f}$ (a generous gift from Dr. Masaaki Komatsu, Tokyo Metropolitan Institute of Medical Science), and Fip200 ${ }^{\mathrm{f} / \mathrm{f}}$ females were crossed with Nestin-Cre males to generate Atg $7^{\text {Nestin }}$ cko and Fip200 $00^{\text {Nestin }}$ cko mice. All animals were maintained in a mixed background between C57BL/6 and 129. All animal experiments were approved by and performed in accordance with guidelines provided by the Institutional Animal Care and Use Committee at St. Jude Children's Research Hospital.

\section{Immunostainings and Histologic Analyses}

Embryonic and newborn mouse brains were dissected in phosphate-buffered saline (PBS) and fixed overnight in $4 \%$ paraformaldehyde (PFA) in PBS at $4^{\circ} \mathrm{C}$. Mice older than postnatal day (P) 1 were transcardially perfused with 4\% PFA and fixed overnight. The brains were then cryoprotected using 30\% sucrose in phosphate-buffered saline (PBS) overnight at $4^{\circ} \mathrm{C}$ and embedded in OCT for cryosectioning. Frozen sections were washed with $0.2 \%$ Triton X-100 in tris-buffered saline (TBST) and incubated in the blocking solution ( $5 \%$ normal goat serum or normal donkey serum in TBST) for $1 \mathrm{~h}$ at room temperature. Sections were incubated with primary antibodies diluted in the blocking solution overnight at $4{ }^{\circ} \mathrm{C}$, washed with TBST, and incubated with Alexa Fluorconjugated secondary antibodies (Invitrogen), diluted at 1:1000 in the blocking solution for $2 \mathrm{~h}$ at room temperature. Sections were mounted in ProLong Gold antifade reagent with DAPI (Invitrogen). Primary antibodies against the following targets were used: NeuN (Synaptic System, 1:500), NeuN (Millipore, 1:1000), Cleaved caspase-3 (Cell Signaling, 1:200), GFAP (Dako, 1:500), Iba1 (Wako, 1:500), P62 (Abnova, 1:500), Ubiquitin (Dako, 1:500), p-S6 (Cell Signaling, 1:400), p-elF2 $\alpha$ (Cell Signaling, 1:100), Chop (Santa Cruz, 1:100), BiP (Abcam, 1:500), and ATF6 (Abcam, 1:500), Calbindin

* Modified with permission. Joo, J.H., Wang, B., Frankel, E., Ge, L., Xu, L., Iyengar, R., Li-Harms, X., Wright, C., Shaw, T.I., Lindsten, T., et al. (2016). The Noncanonical Role of ULK/ATG1 in ER-to-Golgi Trafficking Is Essential for Cellular Homeostasis. Mol. Cell 62, 491-506. 
(Millipore, 1:500), L1 (Millipore, 1:500), NF165 (Developmental Studies Hybridoma Bank, 1:100), TAG-1 (Developmental Studies Hybridoma Bank, 1:5), NCAM (Developmental Studies Hybridoma Bank, 1:100), Satb2 (Abcam, 1:500), Ctip2 (Abcam, 1:200), anti-Tbr1 (Abcam, 1:500), rabbit anti-GFAP (DAKO, 1:500), rabbit antiCalretinin (Millipore, 1:500), rabbit anti-5-SERT (Millipore, 1:1000), guinea pig antiVGluT2 (Millipore, 1:2000).

For histologic analysis, formalin-fixed brains were processed and embedded in paraffin by using standard methods. Sagittal sections $(4 \mu \mathrm{m})$ were cut and stained with hematoxylin and eosin (H\&E), Luxol Fast Blue, and counterstained with Nissl by staff in the St. Jude Veterinary Pathology Core, and the sections were examined by a pathologist blinded to the experimental groups. Fluoro-Jade C (Millipore, Cat\# AG325) staining was carried out on 20- $\mu \mathrm{m}$ frozen sections per the manufacturer's instructions. Quantification of CA1 pyramidal neurons was done with ImageJ software (National Institute of Health, USA). The counts of DAPI in a $500 \mu \mathrm{m}$ region of CA1 were determined manually. For each animal, the average number of neurons was calculated from at least 5 sections. Cell numbers in the mutants were normalized to their corresponding controls.

To quantify the dorsoventral corpus callosum (CC) width, low-magnification images were taken with a Marianas 2 microscope. Using SlideBook 6.0 software, the CC width was measured in 5 rostrocaudal sections per animal, and the values were presented as the mean \pm s.e.m. To quantify the number of axonal bundles crossing the palliumsubpallium boundary (PSPB) at E14.5, a line spaning the.PSPB was drawn, and the number of axonal bundles crossing the line was quantified from 3 comparable sections from each $U l k 1 / 2$-deficient mouse and the control. Imaris was used to count the numbers of different cell types in the brains of control and Ulk1/2-deficient mice, At least three comparable sections from individual control and Ulk1/2-deficient mice were quantified, and values were shown as the mean \pm s.e.m.

\section{Behavioral Analyses}

For the limb-clasping test, mice were suspended by pulling their tails. For the rotarod assay, mice were put on a rotating rod, the speed of which gradually increased from 0 to $40 \mathrm{rpm}$ at a rate of $10 \mathrm{rpm} / \mathrm{min}$. The time recorded was when the mouse fell from the rod, and 5 min was used as the cutoff for this analysis. For the gait analysis, the paws of mice were dipped in nontoxic colored ink: fore paws in blue ink and hind paws in red ink. The mice were then allowed to walk through a tunnel placed on top of white paper. The paw prints were then air dried and scanned.

\section{Morphometric Analyses of Electron Micrographs}

Mice were deeply anesthetized with $\mathrm{CO}_{2}$, perfused transcardially with $10 \mathrm{~mL}$ phosphate buffer followed by $10 \mathrm{~mL} 2.5 \%$ glutaraldehyde $/ 2 \%$ PFA in $0.1 \mathrm{M} \mathrm{CaCO}_{4}$. The brains were removed and fixed in the same fixative and postfixed in $2 \%$ osmium 
tetroxide in $0.1 \mathrm{M}$ sodium cacodylate buffer with $0.3 \%$ potassium ferrocyanide for $2 \mathrm{~h}$. Vibratome sections (100-mm) of the brain were cut sagittally and collected in cold PBS, rinsed in phosphate buffer, dehydrated through a series of graded ethanol-to-propylene oxide solutions, infiltrated and embedded in epoxy resin, and polymerized at $70^{\circ} \mathrm{C}$ overnight. Semithin $(0.5-\mu \mathrm{m})$ sections were stained with toluidine blue for light microscope examination. Ultrathin (80-nm) sections were cut and imaged using an FEI Tecnai F 20 TEM FEG electron microscope with an AT XR41 camera.

For quantitative analysis of endoplasmic reticulum (ER), all images of neurons in the hippocampal CA1 regions of 2 controls and 2 mutants ( 8 -wk-old) were taken at the same parameters. For each sample, images were randomly obtained from at least 20 neurons. For the quantification of ER number and diameter, the TIFF images were opened with NIS-Elements software (Nikon) and calibrated individually. A 2- $\mu \mathrm{m}$ line was drawn to span as many ER tubules as possible. The number of ER tubules the line crossed was recorded as the ER number. The diameters of the ER tubules covered by the line were also measured individually. To quantify the percentage of ER occupation in the cytosol, the same sets of images were opened in the ImageJ software and calibrated accordingly. Then a $25-\mu \mathrm{m}^{2}$ area was drawn in the cytosol where the ER tubules were the most abundant. The ER tubules in each image were selected and masked manually. The total area of ER in the defined cytosolic region was then measured and recorded. Ten cells from each sample were analyzed.

\section{Immunoprecipitation}

Endogenous ULK1 was extracted from the brain of 4-wk-old WT mice or from MEFs by using a Triton-based cell lysis buffer ( $40 \mathrm{mM}$ HEPES, $120 \mathrm{mM} \mathrm{NaCl}, 1 \mathrm{mM}$ EDTA, $1.5 \mathrm{mM} \mathrm{Na}_{3} \mathrm{VO}_{4}, 50 \mathrm{mM} \mathrm{NaF}, 10 \mathrm{mM} \beta$-glycerophosphate, $20 \mathrm{mM} \mathrm{MoO}_{4}, 0.5 \%$ Triton X-100, protease inhibitor, phosphatase inhibitor). The lysates were incubated with anti-ULK1 antibody (Santa Cruz Biotechnology, sc 10900) overnight at $4^{\circ} \mathrm{C}$ and precipitated with Protein $\mathrm{G}$ agarose beads (Thermo Scientific). For immunoprecipitation of GFP-SEC16A, whole-cell extracts were prepared from 293T cells by using the described Triton-based buffer and precipitated with anti-GFP antibody-conjugated sepharose beads (Abcam, ab69314) after overnight incubation at $4^{\circ} \mathrm{C}$. The beads were washed 5 times with cold Triton-based buffer and incubated at $95^{\circ} \mathrm{C}$ for $5 \mathrm{~min}$ in SDS sample buffer (Sigma Aldrich). Phosphatase treatment was performed on GFP IPs by using calf alkaline intestinal phosphatase (Sigma Aldrich) per the manufacturer's protocol, before elution in SDS sample buffer. Anti-FLAG M2-agarose beads (Sigma Aldrich, A2220) were used for immunoprecipitation of FLAG-tagged proteins.

\section{COPII Vesicle-Formation Assay}

The preparation of cytosol (source of soluble COPII proteins) and medium-speed membrane-pellet (source of membrane and cargo) fractions has been described before (Ge et al., 2013; Ge et al., 2014). Each vesicle-budding reaction consists of $3 \mu \mathrm{L}$ 
membrane from the medium-speed pellet (OD600 $=10$ for total membrane), $25 \mu \mathrm{L}$

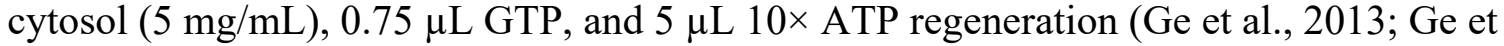
al., 2014). B88 buffer (20 mM HEPES-KOH, pH 7.2, $250 \mathrm{mM}$ sorbitol, $150 \mathrm{mM}$ potassium acetate, and $5 \mathrm{mM}$ magnesium acetate) was added last to adjust the reaction mixture to a final volume of $50 \mu \mathrm{L}$. The reaction was performed at $30^{\circ} \mathrm{C}$ for $1 \mathrm{~h}$ followed by centrifugation at $20,000 \mathrm{xg}$ for $20 \mathrm{~min}$. Supernatant aliquots $(35 \mu \mathrm{L})$ were transferred to an ultracentrifuge tube for sedimentation at 100,000 $\mathrm{xg}$ in a Beckman TLA100.3 rotor for $30 \mathrm{~min}$. The supernatant fractions were removed, and the small membranes were analyzed by immunoblotting with the antibodies indicated in the figures.

\section{Plasmid Constructs}

The pmGFP-SEC16A (Addgene, Cat\# 15775) and pmGFP-SEC16B vectors (Addgene, Cat\# 15776) have been previously described (Bhattacharyya and Glick, 2007). SERT-GFP (Origene Technologies, Rockville, MD, Cat\# RG210187) and VSVG (ts045)-GFP vectors (Addgene, Cat\# 11912) were used in the ER-to-Golgi trafficking assay (Presley et al., 1997). The pCMV6-MYC-DDK-human SEC16A plasmid (Cat\# RC223625), pCMV6-MYC-DDK-human ULK1 (Cat\# RC215643), and pCMV6- MYCDDK-human ULK2 (Cat\# RC206010) were purchased from OriGene Technologies. FLAG-tagged ULK1 and deletion constructs in the pME18S vector (a generous gift from Dr. Toshifumi Tomoda, Beckman Research Institute of City of Hope, Duarte, CA) were previously described (Joo et al., 2011; Yan et al., 1998). The FLAG-tagged ULK2 construct in the PCSII vector was a generous gift from Dr. Do-Hyung Kim (University of Minnesota, Twin Cities, MN). Plasmid vectors, $\mathrm{pCS} 2^{+}$, containing $S E C 24 A, S E C 24 B$, $S E C 24 C$, and $S E C 24 D$ open-reading frames, with a $3 \times \mathrm{HA}$ tag at the $\mathrm{N}$ terminus, were described previously (Kim et al., 2007). The Ulk1 and Ulk1 kinase-inactive (KI) (K46A) mutant, as described previously (Joo et al., 2011), were subcloned into the 5'- or 3'-end of EcoR1-restriction enzyme sites of the MSCV-IGFP-MII (generously provided by Dr. Terrence Geiger, St. Jude) and pcDNA3.1 vectors for retrovirus generation and transient transfection, respectively. Human SEC16A phosphorylation-site mutants were introduced into the pmGFP-SEC16A and pCMV6-entry-(MYC-DDK-tagged) human SEC16A vectors by using mutagenic oligonucleotides.

\section{Generation of Murine Embryonic Fibroblasts, Cell Culture, Transfection, Gene Silencing, and Drug Treatment}

Ulk1-ko, Ulk2-ko, and Ulk1/2-dko murine embryonic fibroblasts (MEFs) were prepared from the E12.5 embryos of $U l k 1^{+/-} ; U l k 2^{+/-} \times U l k 1^{+/-} ; U l k 2^{+/-}$crosses. Embryos were collected, washed twice with PBS, incubated in trypsin, and then separated into single-cell suspensions. Cells were expanded in DMEM (Invitrogen) supplemented with $10 \%$ fetal bovine serum (FBS) (Hyclone), MEM nonessential amino acids (NEAA; Life Technologies), $L$-glutamine (Life Technologies), $\beta$-mercaptoethanol (Life Technologies), and gentamycin (Life Technologies). These primary MEFs were then immortalized using 
FuGENE $^{\mathrm{TM}} 6$ (Roche) to transfect the cells with $1 \mu \mathrm{g}$ of an expression vector expressing SV40, as described previously (Joo et al., 2011).

After transfection, cells were maintained in normal growth media. $\operatorname{Atg} 7^{-1-} \mathrm{MEFs}$ and stably transduced MEFs were generated as described previously (Joo et al., 2011). Briefly, MSCV-IGFP-MII containing no cDNA (empty vector), WT ULK1, or mutant ULK1, and helper vector (generously provided by Dr. Terrence Geiger) were cotransfected into 293T cells by using FuGENE 6. Transfected cells were then incubated in DMEM with $10 \%$ FBS for $48 \mathrm{~h}$. Supernatant was collected twice daily and used to infect GP+E86 retroviral-producer cells in the presence of Polybrene $(8 \mu \mathrm{g} / \mathrm{mL})$. Transduced GP+E86 cells were FACS-sorted by the presence of GFP, and cell-free supernatant was used to transduce $U l k 1^{-/-}$MEFs. MEFs and 293T cells were grown in DMEM supplemented with 10\% FBS, penicillin/streptomycin (Invitrogen), and Glutamax (Invitrogen) at $37^{\circ} \mathrm{C}\left(5 \% \mathrm{CO}_{2}\right)$. The cells were transfected with FuGENE 6 according to the manufacturer's protocol for transient overexpression of cDNA constructs.

Knockdown experiments in MEFs were performed using Lipofectamine RNAi Max (Life Technologies) per the manufacturer's protocol and with the following siRNA constructs obtained from Dharmacon: pooled nontargeting siRNA (D-001810-10-05), SMARTpool: ON-TARGETplus Ulk1 siRNA (L-040155-00-0005); SMARTpool: ONTARGETplus Sec16a siRNA (L058170-02-0005); ON-TARGETplus Sec16a siRNA Targeted Region: 3'UTR (J-058170-11-0005); SMARTpool: ON-TARGETplus Sec24a siRNA (L-056263-01-0005); SMARTpool: ON-TARGETplus Sec24b siRNA (L-04889801-0005); SMARTpool: ON-TARGETplus Sec24c siRNA (L-059052-01-0005); SMARTpool: ON-TARGETplus Sec24d siRNA (L-065430-01-0005); SMARTpool: ONTARGETplus Atg7 siRNA (L-049953-00-0005);SMARTpool: ON-TARGETplus Atg13 siRNA (L-053540-01-0005); and SMARTpool: ON-TARGETplus Atg14 siRNA (L172696-00-0005). Knockdown was confirmed by RT-qPCR (TaqMan) analyses, immunoblot analyses, or both. To chemically induce ER stress, 293T cells were treated with Brefeldin A (Sigma Aldrich) and dissolved in DMSO (final concentration, 10 $\mu \mathrm{g} / \mathrm{mL}$ ) for $6 \mathrm{~h}$. Cells were then lysed and analyzed by immunoblotting.

\section{Immunoblot Analyses and Antibodies}

Whole hippocampi or MEF cells were lysed in Triton-based cell lysis buffer. Proteins in cleared lysates were electrophoretically separated on $4 \%$ to $12 \%$ bis-Tris gels (Life Technologies). Proteins were then transferred to either a nitrocellulose or a PVDF membrane.

After incubation with a 5\% skim milk block, blots were probed with antibodies directed against the following targets: P62 (Sigma Aldrich, Cat\# P0067), LC3 (MBL, Cat\# PM036) ULK1 (Sigma Aldrich, Cat\# A7481), p-ULK1 (S555) (Cell Signaling, Cat\# 5869), p-(Ser/Thr) Phe (Cell Signaling, Cat\# 9631), SEC16A (Novus, Cat\# NB1001799), SEC16A pS846 (custom polyclonal antibody from Rockland), ATG7 (Cell 
Signaling, Cat\# 2631), ATG13 (Sigma Aldrich, Cat\# SAB4200100), p-ATG13 (S318) (Rockland, Cat\# 600-401-C49), ATG14 (MBL, Cat\# PD026), CDC37 (Santa Cruz Biotechnology, Cat\# sc-13129), SERT (Millipore, Cat\# PC177L), ATF3 (NOVUS, Cat\# NBP1-02935), p-elf2 $\alpha$ (Cell Signaling, Cat\# 3597), CHOP and BIP (a generous gift from Dr. Linda Hendershot, St. Jude), GFP (Abcam, Cat\# ab6556), FLAG (Sigma Aldrich, Cat\# F1804), HA (Cell Signaling, Cat\# 3724), Myc (Cell Signaling, Cat\# 2276), DDK (OriGene, Cat\# TA50011-100), ACTB (Santa Cruz Biotechnology, Cat\# I-19), and GAPDH (Sigma Aldrich, Cat\# G9545). Membranes were then incubated with horseradish peroxidase-conjugated secondary antibodies, and bands were detected using chemiluminescence-detection kits (Amersham).

\section{Proteomics}

The mass spectrometric (MS) analysis was performed per an optimized platform, as previously reported (Xu et al., 2009). ULK1-interacting proteins were visualized by performing silver staining (Life Technologies) or SYPRO Ruby protein gel staining (Sigma Aldrich) according to the manufacturer's protocol. Proteins in gel bands were reduced by adding dithiothreitol (DTT) and then alkylated by adding iodoacetamide. The gel bands were washed, dried in a speed vacuum, and rehydrated with a trypsincontaining buffer for overnight proteolysis. The digested peptides were extracted, dried, reconstituted, and loaded onto a capillary reverse-phase C18 column by an HPLC system (Waters ACQUITY UPLC). Peptides were eluted in a gradient, ionized by electrospray ionization, and detected by an in-line mass spectrometer (Thermo scientific LTQ Orbitrap Elite). MS spectra were collected, and the top 20 abundant ions were sequentially isolated for MS/MS analysis. This process was cycled over the entire liquid chromatography gradient. The acquired MS/MS spectra were used to search protein databases to obtain possible peptide matches. All matched MS/MS spectra were filtered by mass accuracy and matching scores to reduce the protein false-discovery rate to less than $1 \%$.

\section{Quantitative Real-Time PCR}

Total RNA of adult hippocampi was extracted with RNeasy Mini Kit (Qiagen) according to the manufacturer's instructions. Total RNA was isolated from cells by using TRIzol Reagent (Life Technologies). The reverse-transcription reaction was carried out using the SuperScript III first-strand synthesis kit (Life Technologies) per the manufacturer's instructions. TaqMan gene-expression assays containing FAM-labeled primer/probe sets specific for Ulk1 (Mm-00437238_m1), Ulk2 (Mm-00497023_m1), Sec16a (Mm00462283_m1), Sec24a (Mm00613960_m1), Sec24b (Mm01313235_m1), $\operatorname{Sec} 24 c\left(\mathrm{Mm} 00655499 \_\mathrm{m} 1\right), \operatorname{Sec} 24 d$ (Mm00660744_m1), and $18 S$ were obtained from Applied Biosystems. The real-time PCR reactions were performed in a total reaction volume of $25 \mu \mathrm{L}$ by using FastStart TaqMan Probe Master (Roche) reagent, and results were analyzed using the ABI 7900 real-time PCR-detection system (Applied Biosystems). Relative expression was normalized to $18 S$ RNA and calibrated to the respective controls. 


\section{Immunofluorescence Microscopy}

To assess SEC24C puncta quantity and ULK1 distribution, MEFs were incubated in complete growth media, fixed in 4\% PFA, permeabilized with digitonin $(100 \mu \mathrm{g} / \mathrm{mL}$ in PBS), blocked in 1\% BSA, and then labeled with one of the following antibodies: antiULK1, anti-SEC24C (Abcam, ab122635), anti-HA or anti-FLAG. Cells were then treated with secondary antibodies conjugated to Alexa-555 or Alexa-647 (Invitrogen). To assess endogenous SEC16A distribution, MEFs were fixed in cold methanol for $15 \mathrm{~min}$; slowly brought to room temperature in PBS; and blocked in PBS, $4 \%$ BSA, and $0.1 \%$ Triton X100. MEFs were then labeled with anti-SEC16A antibody (Bethyl Laboratories, Cat\# KIAA0310), followed by Cy3-conjugated secondary antibody (Jackson Immunoresearch, Cat\# 711-165-152). To evaluate VSVG-GFP and SERT-GFP trafficking, MEFs were fixed and prestained with wheat germ agglutinin and Alexa Fluor 647 conjugate (Life Technologies, Cat\# W32466) and then permeabilized with Triton X-100 $(100 \mu \mathrm{g} / \mathrm{mL}$ in PBS) if additional staining with anti-FLAG antibody was needed.

To quantify SEC24C and GFP-SEC16A puncta, MEFs were imaged using a spinning-disc confocal Zeiss AxioObserver operated by Marianas system (Intelligent Imaging Innovations, Denver $\mathrm{CO}$ ) with a $63 \times$ oil objective, and the number of puncta was analyzed using SlideBook 5.5 software (Intelligent Imaging Innovations, Denver CO). To quantify endogenous SEC16A puncta, MEFs were imaged using a swept-field confocal microscope (Nikon Ti-E) equipped with a Roper CoolSNAP HQ2 CCD camera using a Nikon $60 \times, 1.4$ numerical aperture plan apochromat oil objective lens. Acquisition parameters were controlled by NIS-Elements software, and image analysis was conducted using Metamorph software. Confocal microscopy of ULK1 and GFP-SEC16A colocalization was performed on a Nikon TE2000E2 microscope equipped with a Nikon C2 confocal microscope using 488-nm, 561-nm, and 638-nm DPSS lasers for excitation. Images were acquired using a Nikon $60 \times 1.45$ NA objective and analyzed using the NISElements software. The following filters were used: 515/60, 605/75, and 675/50. VSVGGFP - and SERT-GFP-transfected MEFs were imaged using a spinning-disc confocal Zeiss AxioObserver operated by Marianas system (Intelligent Imaging Innovations, Denver CO) with a $63 \times$ oil objective.

To quantify nuclear localization of CHOP, MEFs were grown in normal growth medium, transiently transfected with VSVG (ts045)-GFP or SERT-GFP, and immunostained with anti-CHOP antibody (Santa Cruz, Cat\# sc-575). At least 30 images were acquired, as described above for each condition. The percentage of cells with nuclear CHOP was obtained from 3 independent experiments. As a positive control, the MEFs were treated with Brefeldin A $(10 \mu \mathrm{g} / \mathrm{mL})$ for $6 \mathrm{~h}$, after which $100 \%$ nuclear localization of CHOP was observed.

\section{Immunofluorescence Microscopy in C. elegans}

C. elegans gonads were dissected on poly-L-lysine-coated slides, frozen in liquid nitrogen, and methanol-fixed at $-20^{\circ} \mathrm{C}$ (Audhya et al., 2005). Tissues were 
immunostained using a polyclonal rabbit antibody directed against $C$. elegans SEC16 (Witte et al., 2011) and subsequently labeled with a Cy3-conjugated anti-rabbit secondary antibody. Proximal oocytes were examined on a Nikon Ti-E swept-field confocal microscope, with a Roper CoolSNAP HQ2 CCD camera and using a Nikon 60× 1.4 numerical aperture plan apochromat oil objective lens. Acquisition was controlled using NIS-Elements software, and image analysis was performed using Imaris Bitplane software. Thirty z-sections at $0.2-\mu \mathrm{m}$ steps were acquired. The number and fluorescence intensities of SEC16 puncta were quantified using the Spots module of Imaris Bitplane in at least 10 animals for each condition. Puncta fluorescence was classified as low-, medium-, or high-intensity thresholds, and the percentage of puncta in each category was compared between $\mathrm{N} 2$ control and unc-51-mutant animals. Immunostaining of rabbit anti-MOD-5 antibody (Jafari et al., 2011) and rabbit anti-5-HT antibody (purchased from Dr. H.W.M. Steinbusch, Maastricht University, Masstricht, The Netherlands) was performed with whole-mount WT worms and mod-5(n314) and unc-51(e369) mutants, as described previously (Jafari et al., 2011; Sze et al., 2000).

\section{Endoglycosidase H Assay}

For monitoring ER-to-Golgi trafficking of SERT, extracts prepared from SERTGFP-transfected MEFs were used for GFP immunoprecipitation. Immunopurified proteins were incubated in the presence or absence of Endo $\mathrm{H}$ enzyme or peptide-Nglycosidase F (PNGase) enzyme (New England Biolabs, Cat\# P0704) for $1 \mathrm{~h}$, eluted from the beads in SDS sample buffer with $\beta$-mercaptoethanol, and separated by SDS-PAGE. SERT was detected by immunoblot analysis. Glycosylated proteins such as SERT and VSVG are Endo H-sensitive (Endo H-S) while in the ER, and become Endo H-resistant (Endo H-R) after ER-to-Golgi trafficking. PNGase treatment results in deglycosylated proteins.

\section{Cell-Surface Biotinylation}

The levels of SERT expression in MEFs or platelet PMs were compared using the membrane-impermeant biotinylation reagent, NHS-SS-biotin (Pierce, Inc., Rockford, IL), as described previously (Brenner et al., 2007; Ziu et al., 2012). Briefly, platelet or MEF pellets were washed twice with ice-cold PBSCM solution (PBS containing $0.1 \mathrm{mmol} / \mathrm{L}$ $\mathrm{CaCl}_{2}$ and $\left.1 \mathrm{mmol} / \mathrm{L} \mathrm{MgCl}_{2}\right)$, incubated with NHS-SS-biotin $(1.5 \mathrm{mg} / \mathrm{mL})$ on ice with very gentle shaking for $20 \mathrm{~min}$, rinsed briefly, incubated with PBSCM containing 100 $\mathrm{mmol} / \mathrm{L}$ glycine on ice for $20 \mathrm{~min}$, and lysed with 1\% SDS-1\% Triton X-100.

Biotinylated PM proteins were recovered from the cell lysates by using streptavidinagarose beads (Pierce, Inc.). After washing the beads with high-salt, low-salt, and 50 $\mathrm{mmol} / \mathrm{L}$ Tris- $\mathrm{HCl}(\mathrm{pH} 7.5)$, the biotinylated proteins were treated with either Endo $\mathrm{H}$ enzyme or PNGase F enzyme (New England Biolabs, Cat\# P0704) for $1 \mathrm{~h}$, eluted from the beads in SDS sample buffer with $\beta$-mercaptoethanol, and separated by SDS-PAGE. SERT was detected by immunoblot analysis. 


\begin{abstract}
Axonal Tracing
Embryonic day (E) 18.5 brains were fixed in 4\% PFA overnight at $4{ }^{\circ} \mathrm{C}$. Small crystals of 1,1'-dioctadecyl-3,3,38,38, tetramethylindocarbocyanine perchlorate (DiI) (Molecular Probes) were inserted into the parietal cortex, somatosensory cortex, or dorsal thalamus (dTh) to trace different axonal tracks. The tissues were then stored in $4 \%$ PFA at $37^{\circ} \mathrm{C}$ for 3 to 4 weeks and cut on a vibratome into $100-\mu \mathrm{m}$ sections. Sections were counterstained with Hoechst 33258 (Molecular Probes) and mounted with 50\% glycerol.
\end{abstract}

\title{
Cytochrome Oxidase Staining
}

Brains were removed and fixed overnight in $4 \%$ PFA at $4^{\circ} \mathrm{C}$. Barrel cortices were flattened on superfrost plus slides and cut tangentially (parallel to Layer IV) into $100-\mu \mathrm{m}$ sections on a vibratome. Free-floating sections were incubated with cytochrome oxidase (CO) reaction solution, which included $0.5 \mathrm{mg} / \mathrm{mL} \mathrm{DAB}, 0.5 \mathrm{mg} / \mathrm{mL}$ cytochrome $\mathrm{C}$ (Sigma), and $40 \mathrm{mg} / \mathrm{mL}$ sucrose in PBS at $37^{\circ} \mathrm{C}$ until staining appeared. Sections were washed 3 times in PBS, mounted with Aqua Polymount (Polysciences), and imaged using bright field light microscopy.

\section{Statistical Analyses}

Statistical analyses were performed using SigmaPlot; significance was assessed by 2-tailed Student's $t$-test or by 1- or 2-factor ANOVA analysis followed by HolmSidak post-hoc analysis. 


\section{CHAPTER 3. THE NONCANONICAL ROLE OF ULK/ATG1 IN ER-TO- GOLGI TRAFFICKING IS ESSENTIAL FOR CELLULAR HOMEOSTASIS*}

The third chapter of my dissertation is the publication from analyzing the phenotype of postnatal brain of $U l k 1 / 2$-cdko mice and the possible underlying mechanisms (Joo et al., 2016). I shared first authorship with Dr. Joung Hyuck Joo, a staff scientist in the lab who completed majority of the in vitro experiments. I performed all the animal experiments, and partially contributed to the biochemical studies. The following texts are quoted from the manuscript.

\section{Mice Lacking Ulk1/Ulk2 Expression in the CNS Have a Distinct Pattern of Neuronal Loss}

"Ulk1 and Ulk2 mRNA are expressed throughout the CNS, with the highest levels in the cerebellum (Ulk1>Ulk2) and hippocampus $(U l k 2>U l k 1)$ (Figure 3-1A). To investigate the role(s) of these kinases in the CNS, we generated mice lacking both Ulk1

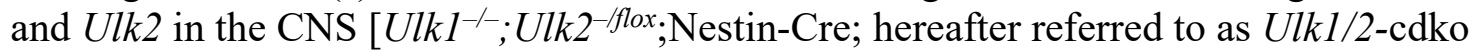
(conditional-double-knockout) mice] and those expressing Ulk1 but not Ulk2 in the CNS $\left(U l k 1^{+/-}\right.$;Ulk2 $2^{-f l o x}$; Nestin-Cre and $U l k 1^{+/+} ; U l k 2^{-f f l o x}$;Nestin-Cre; hereafter referred to as "controls") (Figure 3-1B, C). We confirmed the loss of Ulk1 and Ulk2 mRNA expression in the brains (i.e., hippocampal region) of $U l k 1 / 2$-cdko mice by RT-qPCR analyses (Figure 3-1D). Although the Ulk1/2-cdko mice were born at the expected Mendelian ratio, approximately $40 \%$ died within $24 \mathrm{~h}$ (Figure 3-2A). Survival of the remaining Ulk1/2-cdko mice diminished over the ensuing $12 \mathrm{wk}$, and all were dead by 28 wk (Figure 3-2A). Newborn Ulk1/2-cdko mice were visibly indistinguishable from their littermates, but at 3 wk showed substantial, sex-independent weight loss (Figure 3-2B). The weight of the brains of 8 -wk-old Ulk1/2-cdko mice did not differ from that of littermate controls (Figure 3-2C).

Ulk1/2-cdko mice showed abnormal limb-clasping reflexes (Figure 3-1E) similar to that reported in Atg5-conditional knockout (cko) and Atg7-cko animals (Hara et al., 2006; Komatsu et al., 2006). However, unlike ATG5- or ATG7-deficient mice, which develop cerebellar ataxia (Hara et al., 2006; Komatsu et al., 2006), Ulk1/2-cdko mice had a normal gait (Figure 3-1F) and only a mild delay in motor skill learning compared to littermate controls (Figure 3-1G).

To identify the neurologic deficits in Ulk1/2-cdko animals, we analyzed their

\footnotetext{
* Reprinted with permission. Joo, J.H., Wang, B., Frankel, E., Ge, L., Xu, L., Iyengar, R., Li-Harms, X., Wright, C., Shaw, T.I., Lindsten, T., et al. (2016). The Noncanonical Role of ULK/ATG1 in ER-to-Golgi Trafficking Is Essential for Cellular Homeostasis. Mol. Cell 62, 491-506.
} 
Figure 3-1. Generation and behavioral characterization of $U l k 1 / 2$ cdko mice. (A) In situ hybridization (expression density map) from the Allen Brain Atlas showing the expression of Ulk1 and Ulk2 mRNA in the brains of 8-wk-old mice, with magnified views of the hippocampus and cerebellum. (B) Genomic organization of wild-type and targeted Ulk2 loci. The genomic organizations of the wild-type $\left(U l k 2^{w t}\right)$ and targeted (Ulk2 flox-neo $)$ Ulk2 loci are shown (top 2 diagrams). Mice harboring the targeted Ulk2 allele (Ulk $\left.2^{f l o x-n e o o}\right)$ were crossed with EIIa-Cre transgenic (tg) mice, and progeny harboring $U l k 2^{k o}$ or $U l k 2^{f l l o x}$ alleles (bottom 2 diagrams) were back-crossed with WT mice to eliminate Cre-recombinase expression. (C) Diagram of the generation of Ulk1/2-

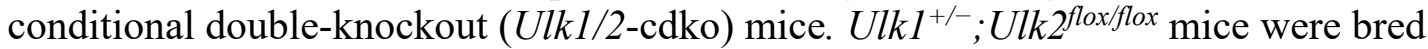

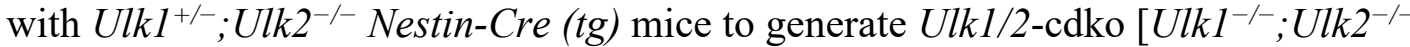
Nestin-Cre (tg)] mice. Controls used in the experiments included $U l k 1^{+/-} ; U l k 2^{-f l o x}$ Nestin-Cre (tg) and Ulk1 ${ }^{+/+} U l k 2^{-f l o x}$ Nestin-Cre (tg) mice. (D) The deletion of Ulk1 and Ulk2 in the cdko mice was verified by RT-qPCR. The levels of Ulk1 and Ulk2 mRNA in the hippocampus of 8-wk-old Ulk1/2-cdko mice $(n=3)$ were normalized to wild-type, age-matched controls $(n=2) . * P<0.001$ (Student's $t$-test). (E) Abnormal limb-clasping reflexes in an 8-wk-old Ulk1/2-cdko mouse (right photograph). When lifted by their tails and slowly lowered toward a horizontal surface, control mice (left photograph) extend their hind limbs and bodies in anticipation of contact. In contrast, Ulk1/2-cdko mice show a pathologic reflex, clasping their fore and hind limbs. (F) The ink paw-print test, in which the forepaws were marked in blue ink and the hind paws in red ink, revealed normal gait patterns in control and Ulk1/2-cdko mice. (G) Motor coordination was tested using a rotarod assay. Control $(n=5)$ and $U l k 1 / 2-c d k o(n=7)$ mice were placed on a rotating rod that was accelerated from 0 to $40 \mathrm{rpm}$ at a rate of $10 \mathrm{rpm} / \mathrm{min}$, and the time spent on the rod was measured. Data shown are the means ( \pm SEM). ${ }^{*} P<0.001$ (Student's $t$-test). (H) Hematoxylin and eosin (H\&E)-stained sections of the cerebellum in 16-wk-old control, Ulk1/2-cdko, and Atg7-cko mice demonstrate the loss of Purkinje cell neurons in the Atg7-cko mice. Intact Purkinje cell neurons are indicated by arrows. Scale bar: $50 \mu \mathrm{m}$. (I) The mean number ( \pm SEM) of Purkinje cell neurons per $200 \mu \mathrm{M}$ in 4month-old control mice $(n=5)$, 4-month-old Ulkl/2-cdko mice $(n=3)$, and 2- to 4month-old $\operatorname{Atg} 7$-cko mice $(n=2)$. ${ }^{*} P<0.001$ (ANOVA). 
A
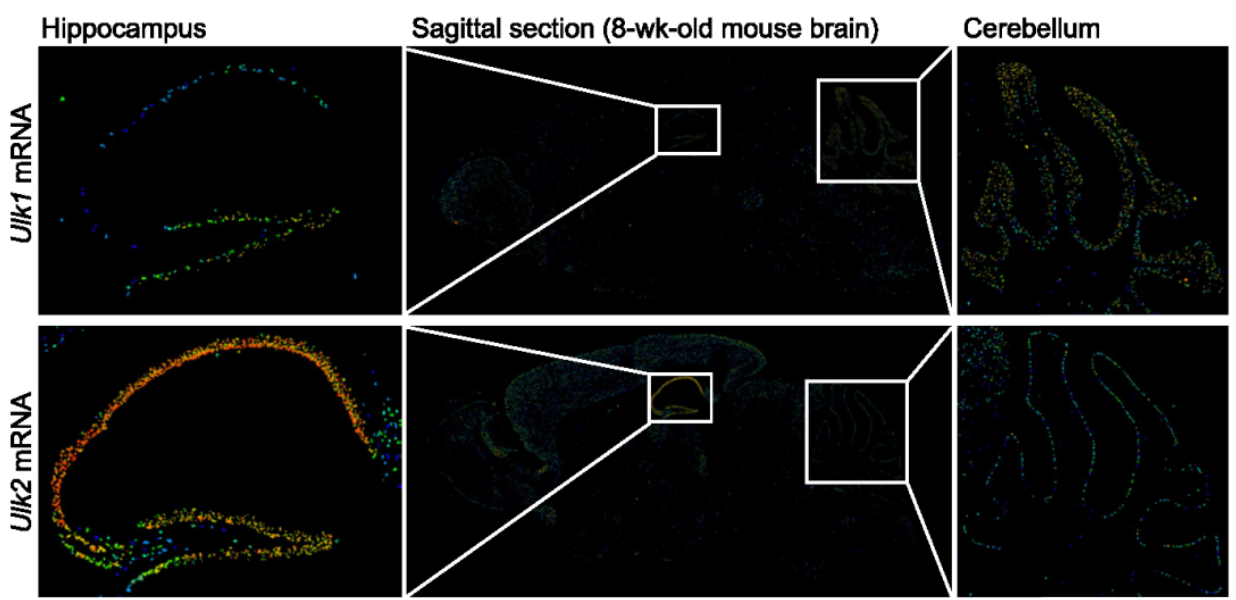
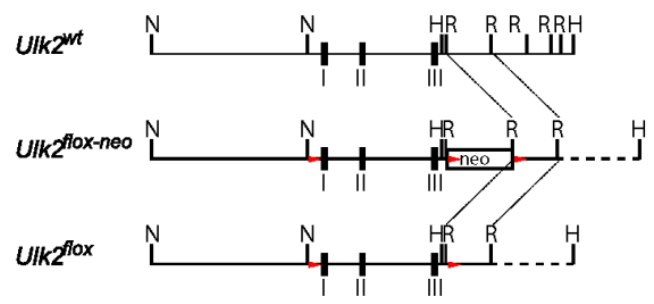

$U / k 2^{k o}$

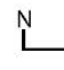

D
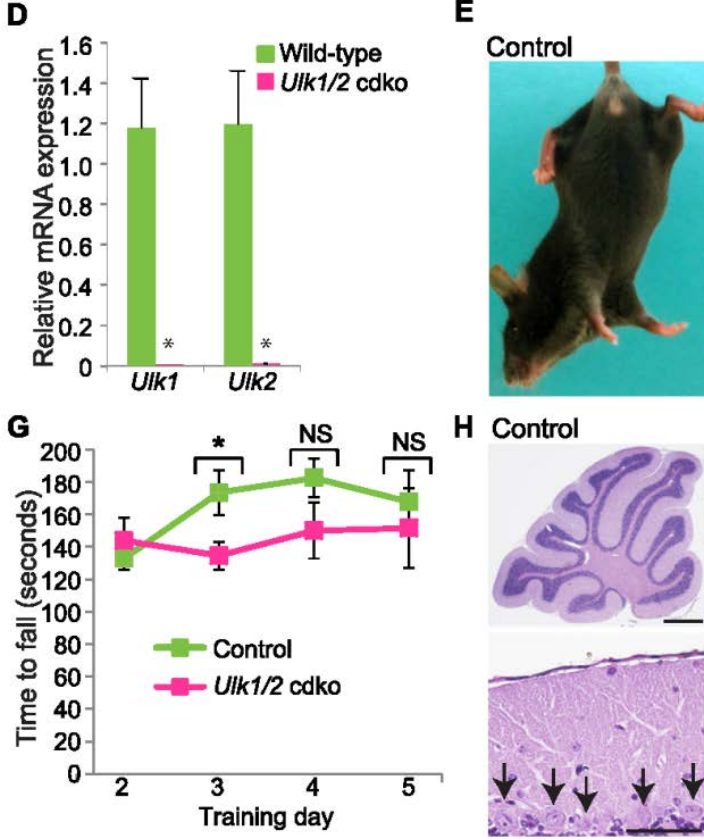

C
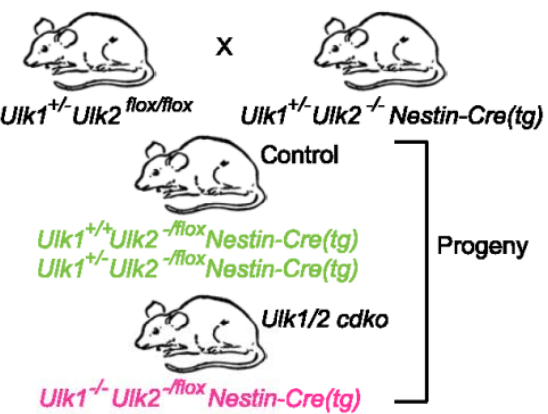

$\underline{1 \mathrm{~kb}}$

F
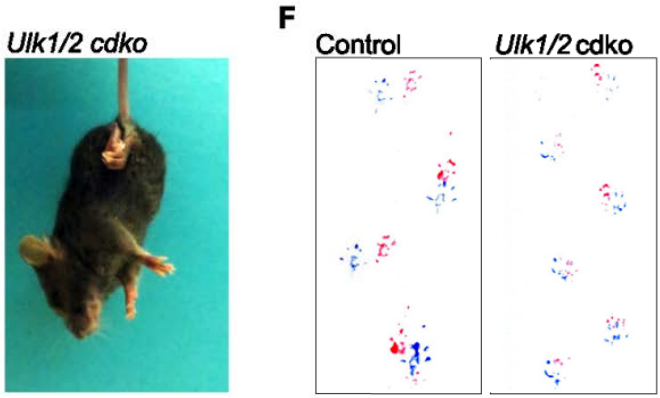

\section{Control \\ Ulk1/2 cdko \\ Atg7cko}
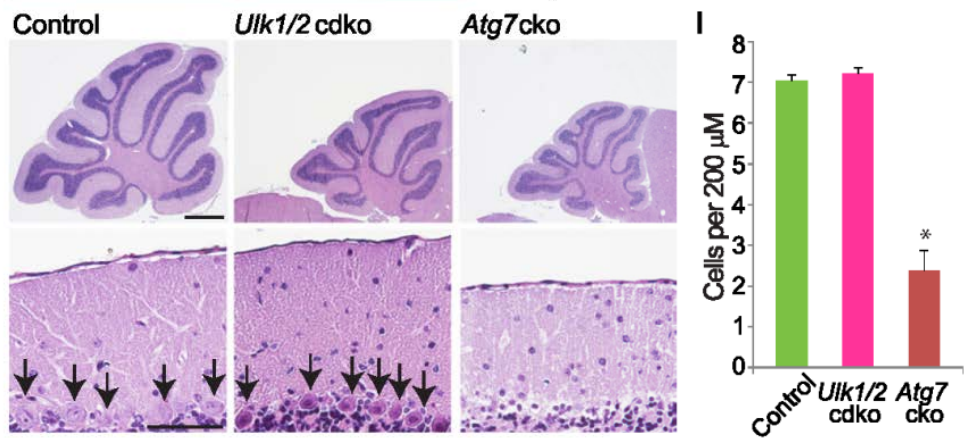
Figure 3-2. Degeneration of pyramidal neurons in the CA1 region of $U L K 1 / 2$ deficient mice.

(A) Survival of control $(n=20)$ and Ulk1/2-cdko $(n=15)$ mice. (B) Average body weight \pm SEM of male (M) and female (F) Ulk1/2-cdko $(n=11)$ mice compared to control $(n=26)$ mice. (C) The average brain weights \pm SEM of control $(n=5)$ and $U l k 1 / 2$-cdko $(n=5)$ mice did not significantly differ (Student's $t$-test). (D) -Representative images of serial brain sections stained with hematoxylin and eosin (H\&E) or DAPI and antibodies against the neuronal marker NeuN. Scale bars: $200 \mu \mathrm{m}$ (E) Average number of pyramidal neurons (normalized to that in littermate controls) \pm SEM in a $500-\mu \mathrm{m}^{2}$ area of CA1 ( $n=3$ mice/genotype for each age group).. ${ }^{*} P<0.001$ (Student's $t$-test) when compared with control. (F) Representative images of brain sections from 16-wk-old mice stained with antibodies against NeuN and the cerebellar Purkinje cell marker, calbindin. Scale bar: $500 \mu \mathrm{m}$. (G) Representative images of Fluoro-Jade C stained and cleaved Caspase 3 (Casp-3) immunostained brain sections from 16-wk-old mice. The CA1 region is indicated by brackets. Scale bars: $200 \mu \mathrm{m}$ (Fluoro-Jade C); $50 \mu \mathrm{m}$ (cleaved Casp-3). (H) Representative images of serial brain sections from hippocampal region of 16-wk-old mice stained with antibodies against GFAP or IBA1 and counterstained with anti-NeuN and DAPI. Scale bars: $50 \mu \mathrm{m}$. 
A

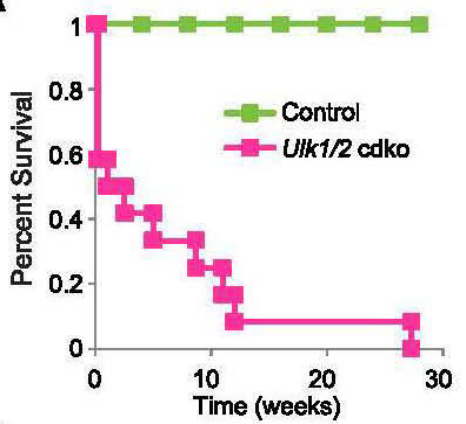

D Control (16 wks) U/k1/2 cdko (3 wk)
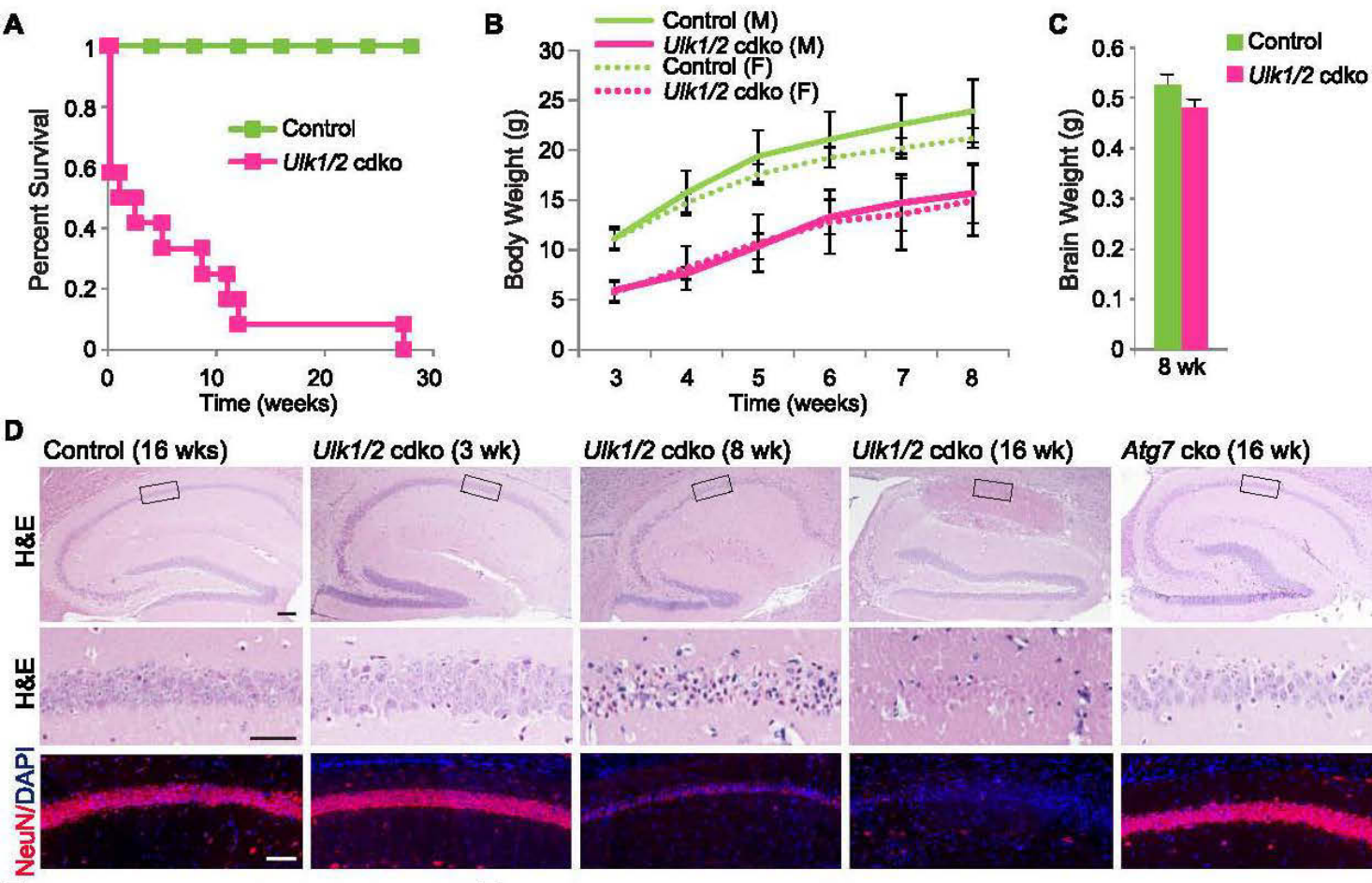

E

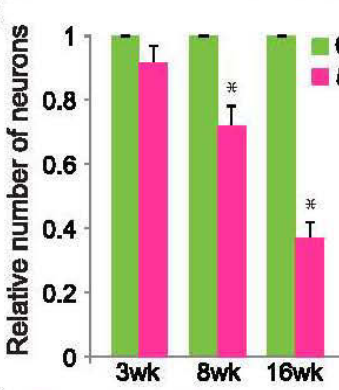

G
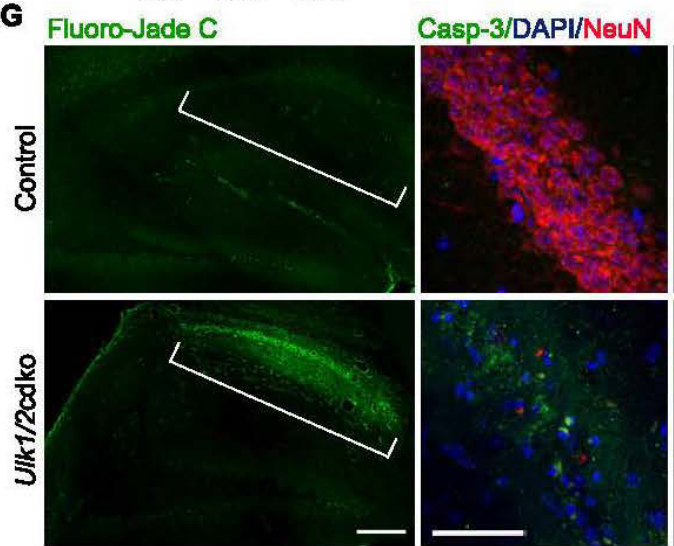

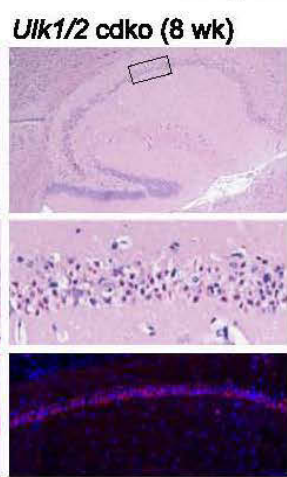

U/k1/2 cdko (16 wk)

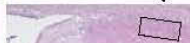

\section{Atg7 cko (16 wk)}
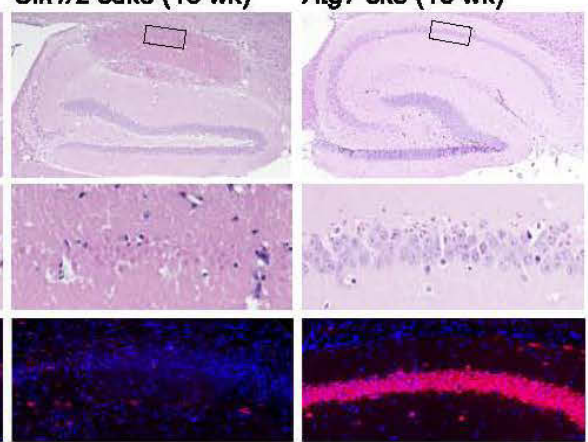

\section{F Control}

Uik1/2 cdko
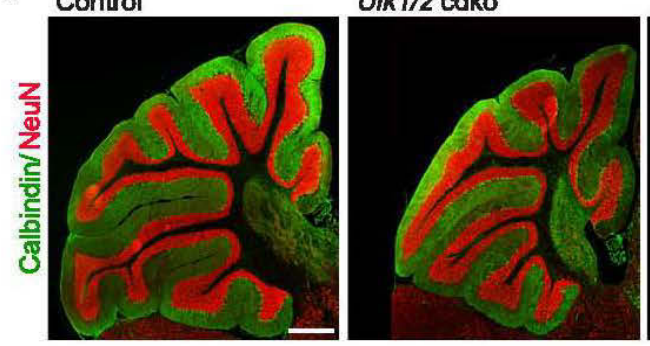

Atg7 dko

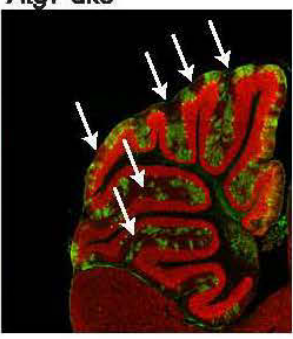

H GFAP/DAPI/NeuN Iba1/DAPI/NeuN
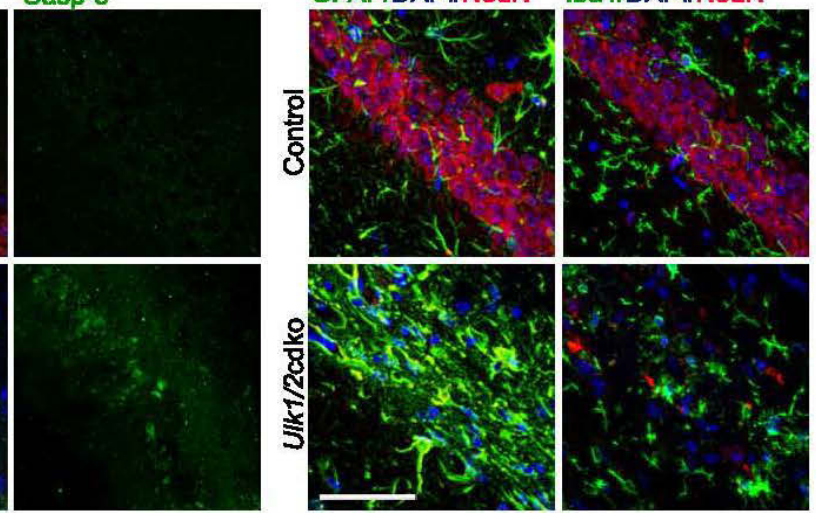
brain histology at various time points. Hematoxylin and eosin staining showed progressive degeneration of pyramidal neurons in the hippocampal CA1 region after $3 \mathrm{wk}$ (Figure 3-2D, E). The dystrophic hippocampal neurons also showed loss of expression of the neuronal marker NeuN (Figure 3-2D). The hippocampal degeneration in these mice was more severe than that in age-matched Atg7-cko mice (Figure 3-2D). In contrast, neuronal loss from other brain regions (e.g., cerebellum) was more severe in Atg7-cko mice than in $U l k 1 / 2$-cdko mice (Figures 3-2F and Figure 3-1H, I). The hippocampal degeneration in Ulk1/2-cdko mice was accompanied by increased fluoro-Jade $\mathrm{C}^{+}$ staining, which labels degenerating neurons, and activated caspase- 3 staining in the CA1 region (Figure 3-2G). Immunostaining for the glial marker GFAP and microglial marker IBA1 was increased in the hippocampal CA1 region (Figure 3-2H), which was consistent with glial activation in response to neuronal damage. These histopathologic changes were not seen in littermate controls. These results highlight the functional redundancy of ULK1 and ULK2 in the CNS and the differential sensitivity of various neuronal populations to their loss.

\section{ULK1/2 Deficiency Activates the Unfolded Protein Response in Hippocampal Neurons}

To determine whether the hippocampal degeneration in Ulk1/2-cdko mice is associated with defective autophagy, we examined steady-state levels of the ubiquitinbinding protein P62/SQSTM1 (hereafter referred to as P62), an autophagy substrate whose levels correlate inversely with autophagy flux (Ichimura and Komatsu, 2010). we did not detect any $\mathrm{P}^{+} 2^{+}$or ubiquitin ${ }^{+}$inclusions in the hippocampal neurons of $U l k 1 / 2-$ cdko mice (Figure 3-3A). These results indicate that in neurons, the autophagy-mediated turnover of ubiquitinated proteins that occurs under basal physiologic conditions proceeds in the absence of ULK1 and ULK2, and that defective autophagy is probably not the primary cause of neuronal degeneration in Ulk1/2-cdko mice.

To learn the cause of the neuronal degeneration in Ulk1/2-cdko mice, we examined the hippocampal region by transmission electron microscopy. Although we did not detect any accumulation of the atypical membranous structures or abnormal mitochondria that are seen with deficiency of other autophagy-related genes (Hara et al., 2006; Komatsu et al., 2006; Liang et al., 2010), the ER compartment was expanded (Figure 3-3B, C). Because ER expansion (Schuck et al., 2009) and mTOR inhibition in neurons (Di Nardo et al., 2009) can result from ER stress and activation of the unfolded protein response (UPR) pathway, which may ultimately lead to cell death, we examined the expression of markers associated with UPR activation in hippocampal pyramidal neurons. Three major sensors of ER stress initiate the UPR: PERK, ATF6, and IRE1. These sensors are activated upon release from the ER chaperone BiP, which is competed away by accumulation of misfolded proteins in the ER (Walter and Ron, 2011). We did not detect spliced XBP1, an alternative product that is produced by activating IRE1 in mRNA samples prepared from the CA1 region. However, the levels of phosphorylated eIF2 $\alpha$, a substrate of PERK, and nuclear localization of ATF6 were both increased in the CA1 region of $U l k 1 / 2$-cdko mice with degenerating neurons (Figure 3-3D, E). 
Figure 3-3. Ulk1/2 deficiency in hippocampal neurons is associated with activation of the UPR pathway.

(A) Representative images of brain sections from 4-wk-old Atg7-cko, 8-wk-old Ulk1/2cdko and 8-wk-old control mice stained with antibodies against ubiquitin and P62, and counterstained with DAPI. Scale bars: $200 \mu \mathrm{m}$. (B) Representative electron micrographs of neurons in CA1 region from 8-wk-old mice. Scale bars: $1 \mu \mathrm{m}$. (C) Morphometric analyses of electron micrographs of hippocampal CA1 neurons from 8-wk-old control $(n=2)$ and $U l k 1 / 2$-cdko $(n=2)$ mice. ${ }^{*} P<0.001$ (Student's $t$-test). (D-F) Representative images of brain sections from the hippocampal region of16-wk-old mice stained with antibodies against p-eIF2 $\alpha$, ATF6 or CHOP. Sections were counterstained with antiNeuN and DAPI. Scale bars: $50 \mu \mathrm{m}$. (G) Diagram of the UPR pathway, highlighting the activation of the PERK and ATF6 arms in the Ulk1/2-cdko mice. 


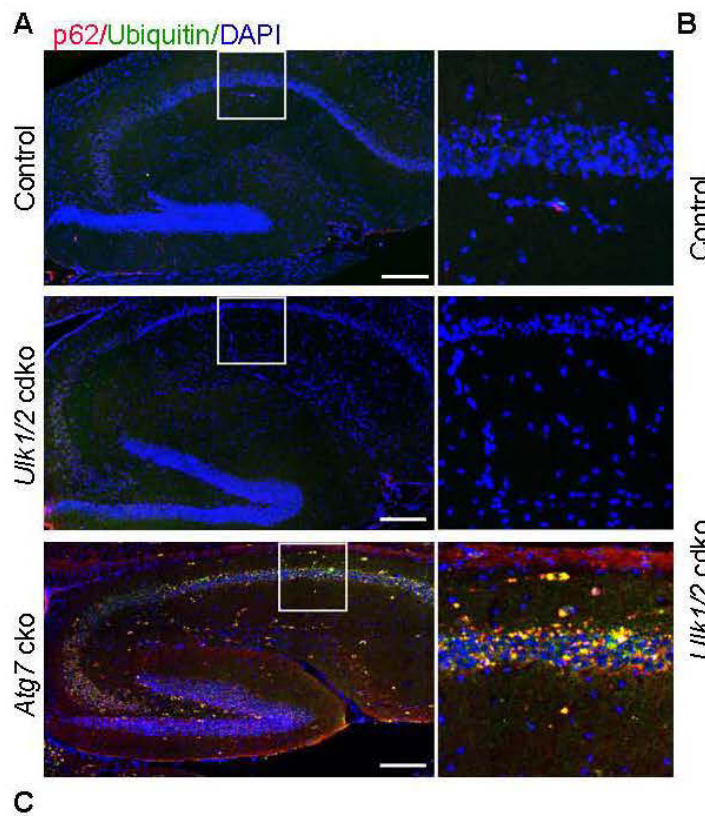

B
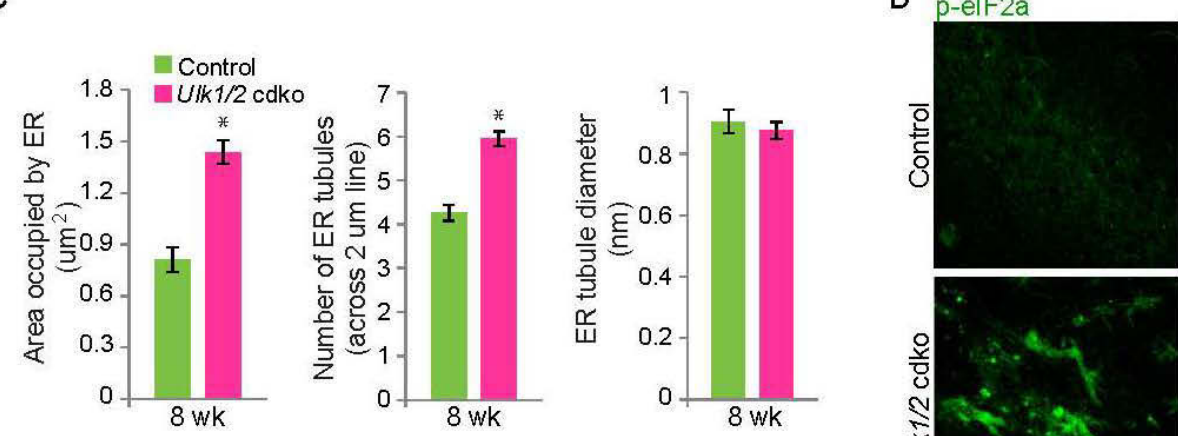

p-elF2a/NeuN/DAPI
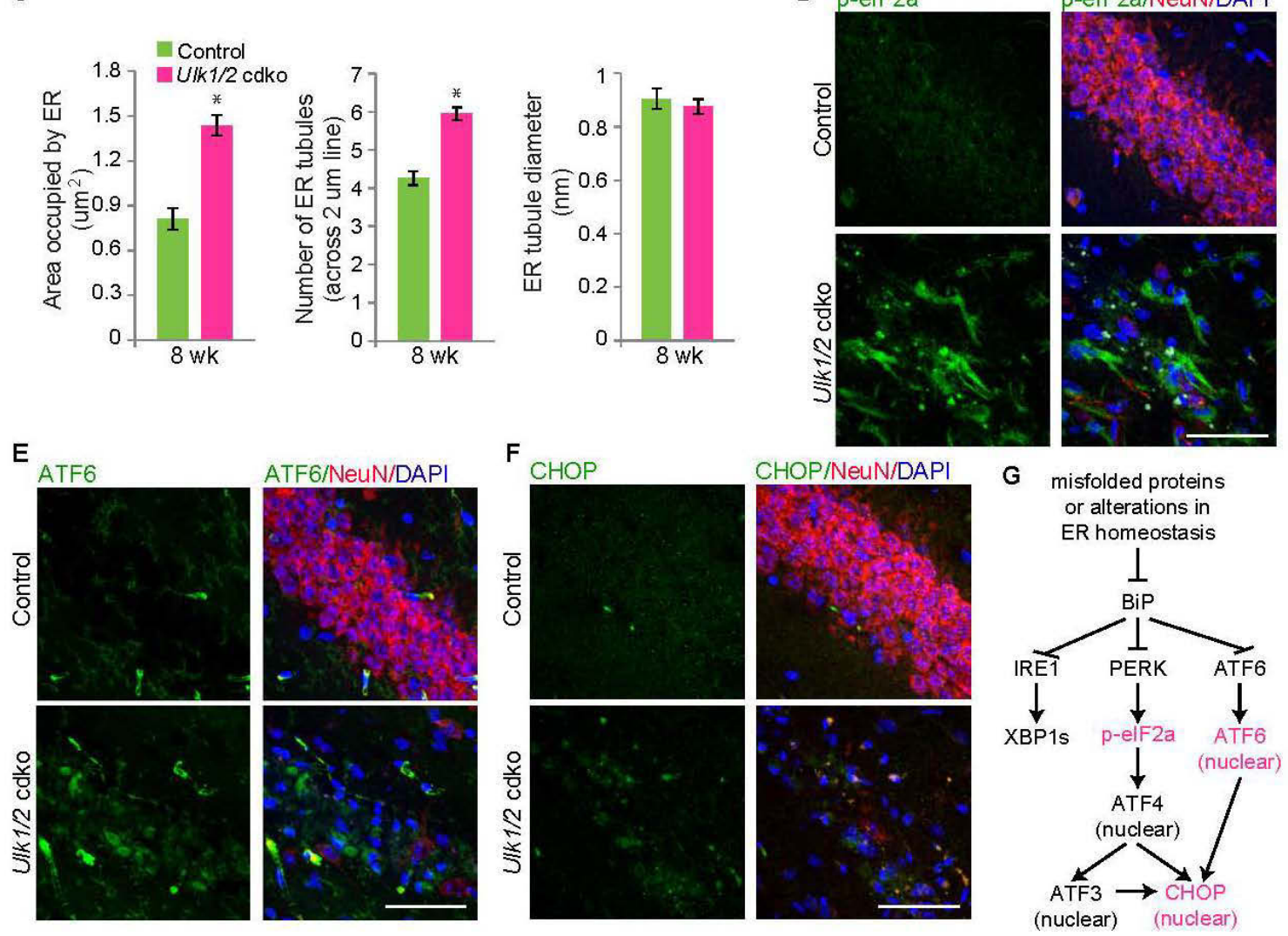
Consistent with activation of the PERK-eIF2 $\alpha$ and ATF6 pathways in the CA1 region of Ulk1/2-cdko mice, the levels and nuclear localization of CHOP, a transcription factor that promotes ER stress-mediated apoptosis, were increased (Figure 3-3F, G). These results indicate that the degeneration of Ulk1/2-deficient pyramidal neurons in the CA1 region disrupts a process that leads to ER stress and UPR activation but not the accumulation of $\mathrm{p} 62^{+} /$ubiquitin ${ }^{+}$inclusions or membranous structures seen in mice lacking other autophagy genes (e.g., Fip200, Atg5, or Atg7).

\section{ULK1 and ULK2 Mediate the Phosphorylation of SEC16A}

We used an unbiased proteomics-based approach to identify ULK/ATG1interacting proteins and gain insight into ULK/ATG1 function. We analyzed immunoprecipitates (IPs) of endogenous ULK1 from WT MEFs by liquid chromatography/mass spectrometry. SEC16A was among proteins identified only in ULK1-containing samples. The ULK1-SEC16 interaction was confirmed by immunoblot analyses of ULK1 IPs in WT MEFs (Figure 3-4A) and hippocampal extracts from WT mice (Figure 3-4B). SEC16A is a large protein that localizes to ER-exit sites (ERES) and facilitates the recruitment of soluble subunits of the coatomer protein complex II (COPII), including SEC23, SEC24, SEC13, and SEC31 (Miller and Barlowe, 2010). The COPII machinery distinguishes transmembrane and luminal secretory cargo from resident ER proteins and packages them into transport vesicles destined for Golgi. Defects in ERto-Golgi trafficking can lead to the accumulation of protein in the ER and activation of the UPR (Fang et al., 2015; Preston et al., 2009); therefore, we further characterized the interaction between the ULKs and SEC16A.

ULK1 exists in a complex with ATG13, FIP200, and ATG101; the stability of the complex and ULK1 levels are reduced in ATG13-deficient cells (Hosokawa et al., 2009). RNAi-mediated silencing of Atg 13 expression reduced ULK1 steady-state levels but did not diminish the ULK1-SEC16A interaction (Figure 3-4C). Given the high degree of homology between ULK1 and ULK2 in their N-terminal domains (Mizushima et al., 2011), it was not surprising that we detected MYC-DDK-tagged ULK2 in GFPSEC16A ${ }^{+}$IPs (Figure 3-4D). These results suggest that the interaction between the ULKs and SEC16A does not require ATG13.

Many ULK-interacting proteins are phosphorylated by ULK1 and ULK2. Therefore, we wondered if SEC16A is an ULK substrate. To test this hypothesis, we used a phospho-serine/threonine-specific antibody that recognizes phosphorylated

serine/threonine residues with tyrosine, tryptophan, or phenylalanine at the -1 position or phenylalanine at the +1 position [hereafter referred to as $\mathrm{p}(\mathrm{S} / \mathrm{T}) \mathrm{Phe}$ ] (Kalabis et al., 2006). This antibody is predicted to recognize the S15 (MQVSFVCQ) and S30 (LDTSFKILD) residues of Beclin-1 and S249 (ILKSFELVK) of VPS34 that are phosphorylated by ULK1 (Egan et al., 2015; Russell et al., 2013). GFP-SEC16A coexpressed with ULK1 or ULK2 but not with the kinase-inactive (KI) ULK1 mutant (K46A) showed robust serine phosphorylation (Figure 3-4D, E). These results indicate that SEC16A is phosphorylated in an ULK1/2-dependent manner. 
Figure 3-4. ULKs mediate the phosphorylation of SEC16A and facilitate in vitro budding of COPII vesicles.

(A) Representative immunoblot analyses of IPs from WT MEFs transfected with either control nontargeting siRNA (Ctrl) or Secl6a siRNA. IPs were performed using antiULK1 antibodies in the presence or absence of epitope-specific blocking peptides (BP).

(B) Representative immunoblot analyses of ULK1 IPs from the hippocampus of 4-wk-old WT mice. (C) Representative immunoblot analyses of ULK1 IPs from WT MEFs transfected with either control nontargeting siRNA (Ctrl) or Atg13 siRNA. (D) Representative immunoblot analyses of 293T cells transfected with GFP-SEC16A and either C-terminal-MYC-DDK-tagged ULK1 or ULK2 expression constructs. Phosphorylated SEC16A was detected using the anti-p(S/T)Phe antibody. (E) Representative immunoblot analyses of GFP IPs from 293T cells transfected with GFP or GFP-SEC16A and untagged WT or KI ULK1 expression constructs. (F) Representative low- and high- magnification pseudocolored images of Ulkl-ko MEFs cotransfected with ULK1 and GFP-SEC16A and stained with antibodies against ULK1. Sections were counterstained with DAPI. (G) Line scans showing the degree of colocalization between ULK1 (red) and SEC16A (green) in lines (not shown) drawn within the boxes labeled 1-4 in the high magnification images from panel F. Scale bars: $10 \mu \mathrm{m}$ (low magnification) and $5 \mu \mathrm{m}$ (high magnification). (H) Diagram of the in vitro COPII-budding reaction. (I) Representative immunoblot analyses of budded vesicles from in vitro COPII-budding reactions using cytosolic fractions from WT or Ulk1-ko MEFs and membrane fractions from Ulk1-ko MEFs. RPN1 is an ER-resident protein; LMAN1/ERGIC53 and SEC22B are COPII cargo whose incorporation into budded vesicles is inhibited by SAR1H79G, a dominant-negative form of the COPII-specific GTPase. (J) Representative immunoblot analyses of budded vesicles from in vitro COPII-budding reactions using cytosolic fractions from $293 \mathrm{~T}$ cells transfected with empty vector (-), WT, or KI ULK1 combined with membrane fractions from untransfected $293 \mathrm{~T}$ cells. 
A

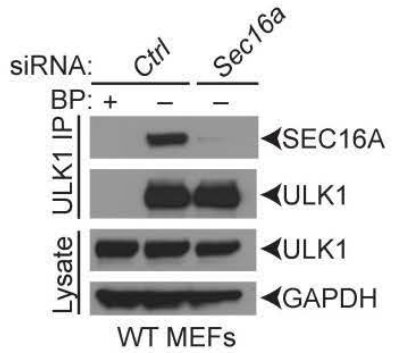

D

GFP: ++--

GFP-SEC16A: - - + + +

ULK: $12-12$

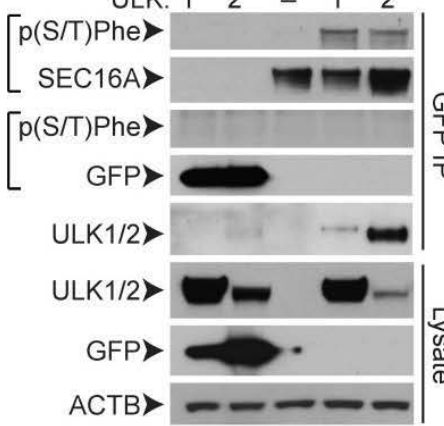

H

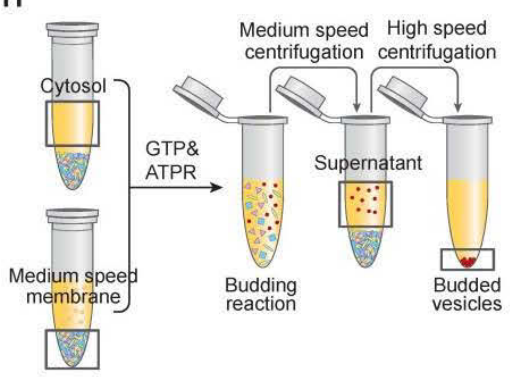

I

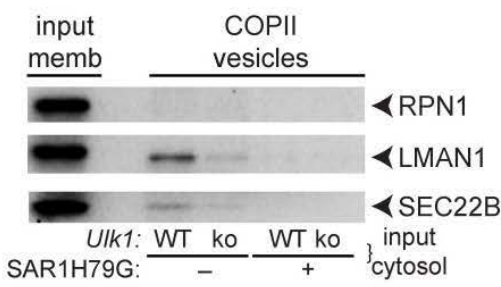

E

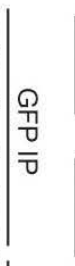

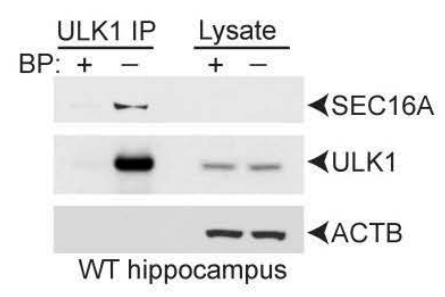

GFP: -++GFP-SEC16A: - - - + +

ULK1:WT - WT KI WT
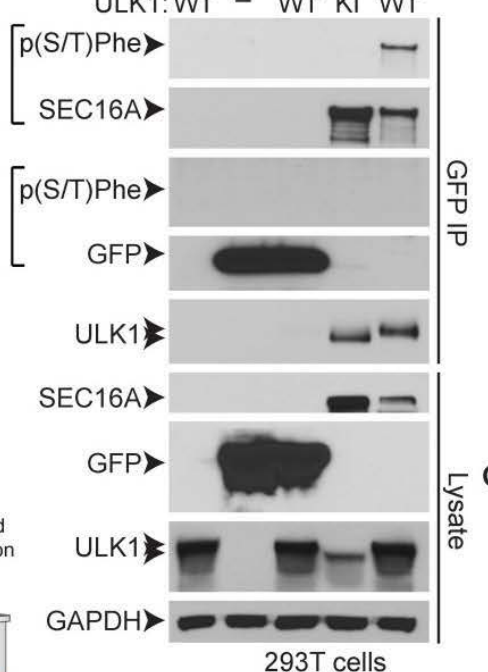

$J$
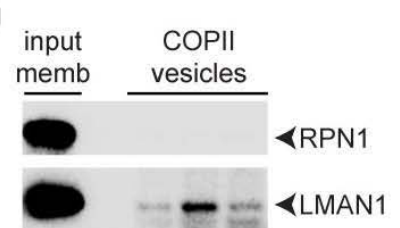

$\mathbf{F}$

C SiRNA: Ctrl Atg13

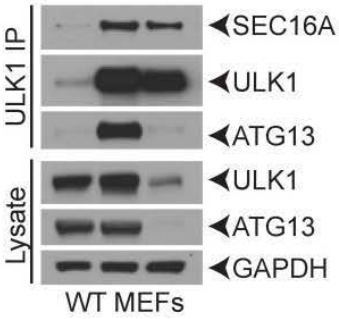

ULK1/GFP-SEC16A/DAPI (Merge)
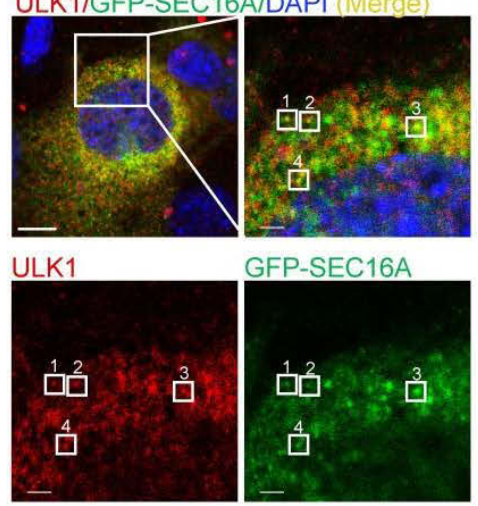

G
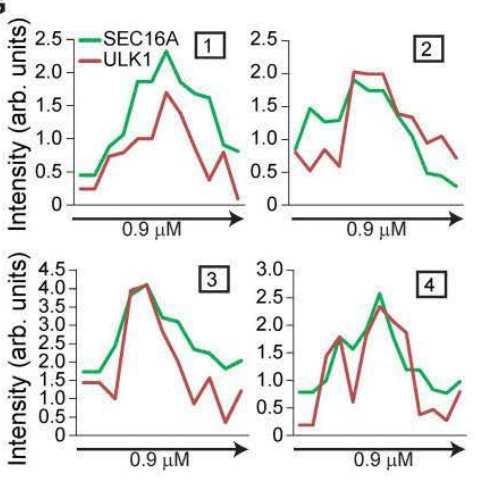

4 SEC22B

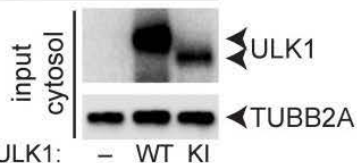




\section{ULK1 Activity Regulates the Budding of COPII Vesicles in Vitro}

The assembly and budding of COPII vesicles is initiated upon activation of the small COPII GTPase SAR1. Sar1 promotes the assembly of soluble SEC23-SEC24 heterodimers, which form the inner coat of the transport vesicles, and soluble SEC13SEC31 heterodimers, which form an outer lattice (Zanetti et al., 2012). SEC16A has a punctate-distribution pattern in cells in culture, consistent with its localization to ERES, where it facilitates the assembly of the soluble COPII components (Bhattacharyya and Glick, 2007; Watson et al., 2006). ULK1 localized to a subset of GFP-SEC16 ${ }^{+}$puncta (Figure 3-4F, G), suggesting that it is recruited to ERES, where it may regulate COPII transport.

We examined the contribution of ULK1 activity in the budding of COPII vesicles in vitro. The assay combines the cargo-containing membrane fraction from 1 cell source with cytosolic extracts, which supply soluble COPII proteins and putative regulatory proteins, from another cell source (Figure 3-4H). Cytosolic extracts derived from autophagy-deficient Atg5-ko MEFs support COPII budding in vitro; and the incorporation of 2 endogenous cargo, SEC22B and LMAN1, into budded COPII is not inhibited by adding the type-III PI3 kinase inhibitor 3-methyladenine (Ge et al., 2014), indicating that essential components of the autophagy machinery are not involved in the budding reaction. In contrast, budded COPII (i.e., SAR1-dependent) vesicles containing SEC22B and LMAN1 were reduced in reactions containing cytosolic extracts derived from Ulk1-ko MEFs (Figures 3-4I). These results suggest a role for ULK/ATG1 activity in the budding of COPII vesicles and/or incorporation of cargo into COPII vesicles.

\section{ULK1 and Related Kinases Have a Conserved Role in Assembling COPII Components at ERES}

Because SEC16A promotes the assembly of soluble COPII components at ERES, and ULK1 localized to a subset of SEC16A ${ }^{+}$puncta in MEFs and was required for efficient formation of LMAN1 and SEC22B-containing vesicles in vitro, we wondered if ULK1 and homologs regulate the assembly of COPII components at ERES. First, we examined the staining pattern of endogenous SEC16 in oocytes from loss-of-function mutant unc-51(e369) Caenorhabditis elegans. The proportion of high-intensity SEC16 ${ }^{+}$ ERES in unc-51 mutant oocytes was significantly lower $(P<0.05)$ than that in WT controls (Figure 3-5A, B). The amount of SEC13 in high molecular-weight gel-filtration fractions, which contain fully assembled COPII complexes comprising SEC13-containing heterotetramers and SEC23-containing heterodimers, was also lower $(P<0.05)$ (Figure 3-5C). These results indicate that UNC-51/ATG1 is involved in the proper assembly of ERES in C. elegans.

The proportion of high-intensity SEC16A ${ }^{+}$ERES in Ulk1/2-dko MEFs (Figure 3-5D, E) was significantly reduced $(P<0.05)$, similar to that in unc-51-mutant oocytes (Figure 3-5A, B). Silencing Sec16a expression in mammalian cells decreases the accumulation of soluble COPII components at ERES (Watson et al., 2006). Given the 
Figure 3-5. ULKs regulate the assembly of COPII complexes at ERES. (A) Representative low- and high-magnification images of distal gonads from WT and unc-51-mutant $C$. elegans stained using a fluorescently labelled anti-SEC16 antibody. (B) Mean percentages \pm SEM of ERES that fall within specified intensity thresholds of SEC16 staining. For each condition, at least 1000 unique ERES from C. elegans gonads were examined. Scale bars: $10 \mu \mathrm{m}$. ${ }^{*} P<0.05$ (Student's $t$-test) when compared with WT. (C) Mean percentages ( \pm SEM) of total SEC13 in peaks 1-3 from Superose 6 gel filtration experiments similar to the one shown in Figure S4A. ${ }^{*} P<0.05$ (Student's $t$-test) when compared with WT. (D) Representative images of endogenous SEC16A immunostaining in WT, Ulk1-ko, Ulk2-ko, and Ulk1/2-dko MEFs. Scale bars: $10 \mu \mathrm{m}$. (E) Mean percentages $( \pm$ SEM) of all ERES that fall within specified intensity thresholds of SEC16A staining. ${ }^{*} P<0.001$ and ${ }^{\#} P<0.05$ (ANOVA) when compared with WT. (F) Mean number $( \pm \mathrm{SEM})$ of SEC $24^{+}$puncta per cell in WT MEFs transfected with the indicated HA-tagged SEC24 isoform (A, B, C, or D) and control nontargeting (Ctrl), Ulk1, or Secl6a siRNA. Ten $\mathrm{HA}^{+}$cells per population were scored. Red arrow highlights the decrease in SEC24C puncta number in Ulk1-depleted cells. ${ }^{*} P<0.001$ and ${ }^{\#} P<0.05$ (ANOVA) when compared with siCtrl. (G) Mean number $( \pm \mathrm{SEM})$ of SEC $24 \mathrm{C}^{+}$puncta per cell in WT, Ulk1-ko, Ulk2-ko, and Ulk1/2-dko MEFs. Ten cells per population were scored. $* P<0.001$ (ANOVA) when compared with WT. (H) Representative images of endogenous SEC24C staining in WT and Ulk1-ko MEFs. Scale bars: $10 \mu \mathrm{m}$. (I) Mean number $( \pm \mathrm{SEM})$ of SEC24C ${ }^{+}$puncta per cell in Ulk1/2-deficient (i.e., Ulk1 ko and Ulk2 shRNA) MEFs stably transduced with the indicated viral vector [i.e., (-) empty vector; WT ULK; or KI ULK1 mutant]. Ten cells per population were scored. $* P<0.001$ (ANOVA) when compared with empty vector-transduced cells. (J) Mean number $\left( \pm\right.$ SEM) of SEC $24 \mathrm{C}^{+}$puncta per cell in WT MEFs transfected with the indicated siRNA. Ten cells per population were scored. ${ }^{*} P<0.001$ (ANOVA) when compared with $C t r l$ siRNA-transfected cells. 

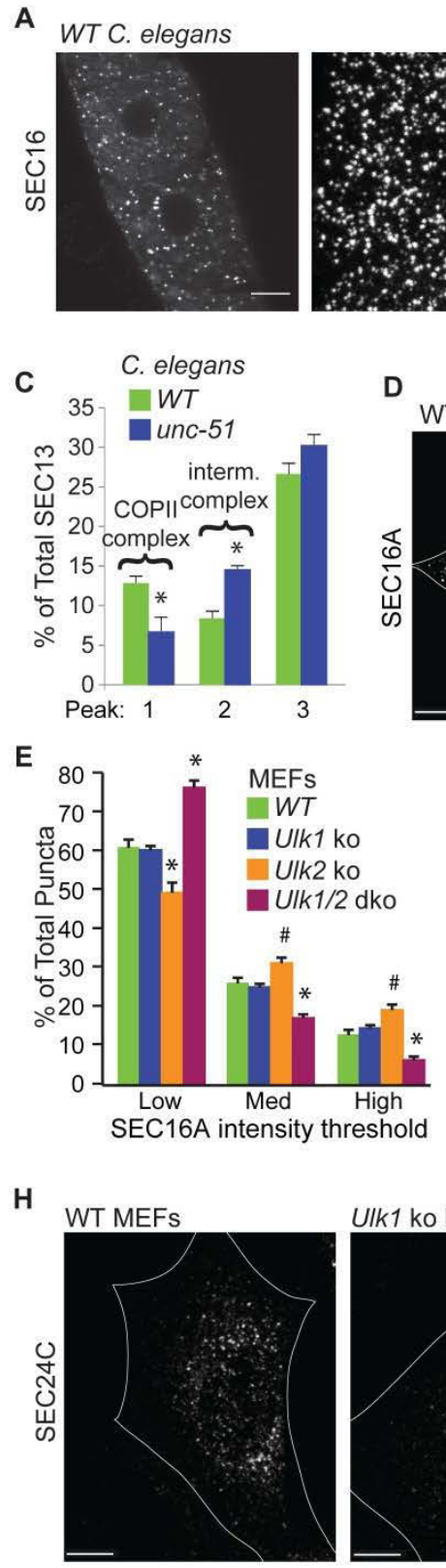

D

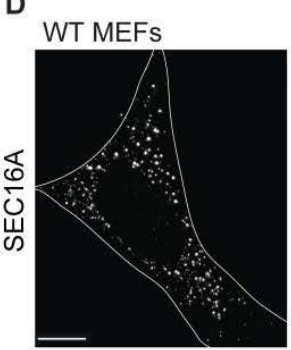

F
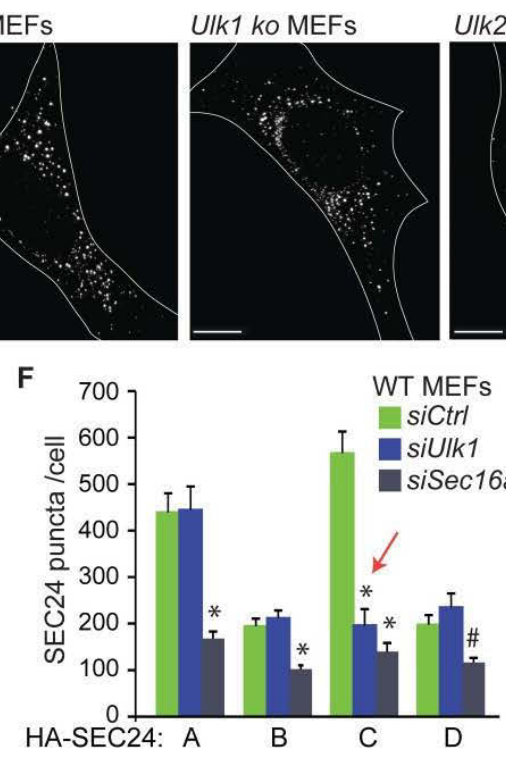

B
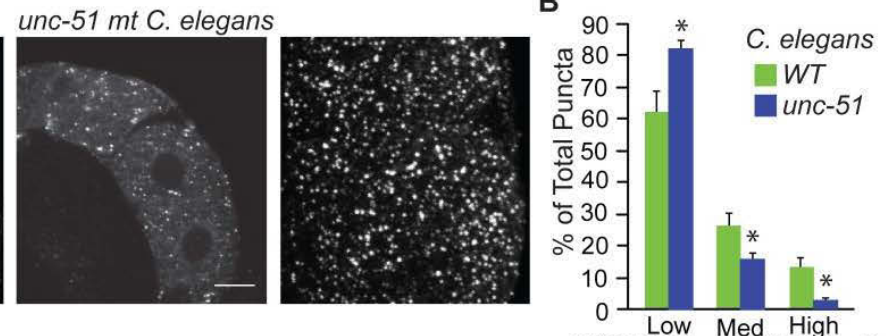

SEC16 intensity thresholds
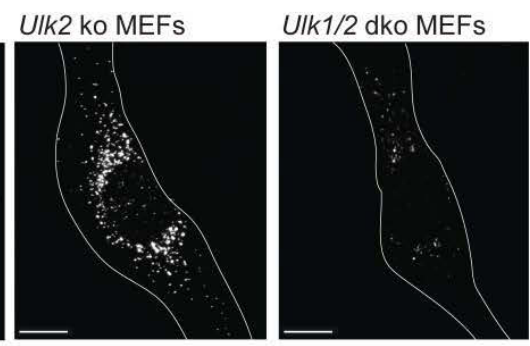

G
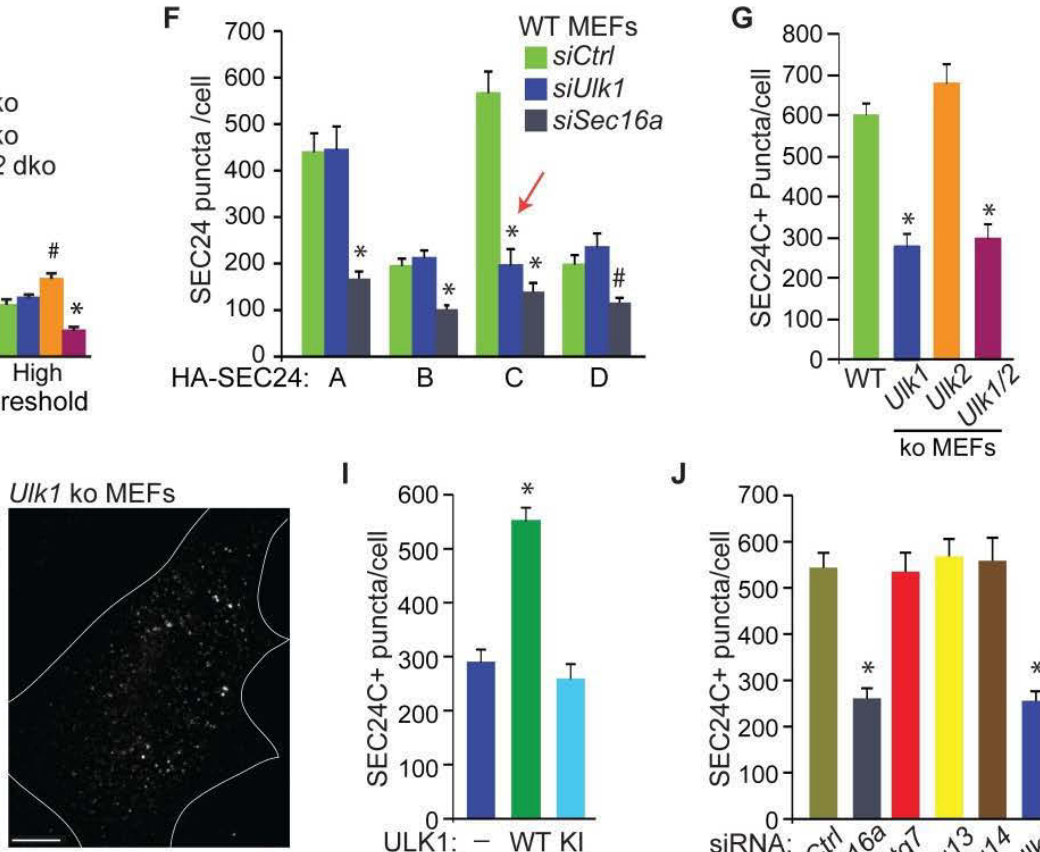
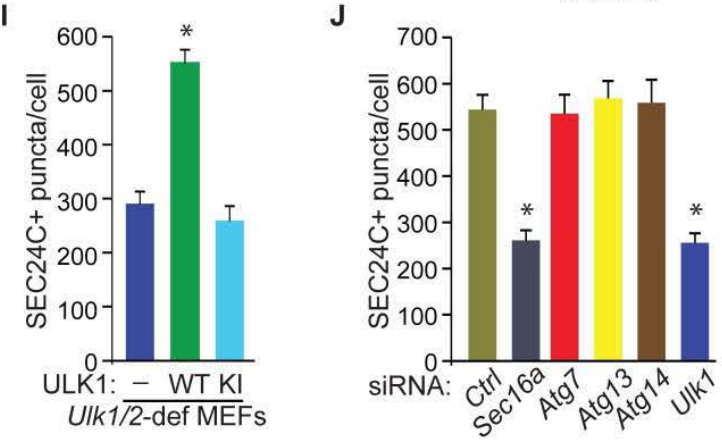
reduced number of high-intensity SEC16A $\mathrm{A}^{+}$puncta in $U l k 1 / 2$-deficient MEFs, we compared the effects of silencing Sec16a and Ulk1 in WT and Ulk2-ko MEFs on the accumulation of cargo adapters, SEC24 A-D, at ERES (Figures 3-5F). RNAi-mediated knockdown of Sec16a significantly reduced $(P<0.001)$ the number of puncta formed by each of the overexpressed SEC24 proteins in MEFs (Figures 3-5F). To our surprise, RNAi-mediated knockdown of Ulk1 in WT or Ulk2-ko MEFs selectively reduced the number of SEC24C $\mathrm{C}^{+}$puncta (Figures 3-5F). The number of endogenous SEC24C ${ }^{+}$ puncta was also significantly reduced $(P<0.001)$ in $U l k 1$-ko and $U l k 1 / 2$-dko MEFs (Figure 3-5G, H), which is consistent with impaired assembly of SEC24C-containing COPII complexes at ERES in cells lacking ULK1. The defect in ERES assembly in either Ulk1- or Ulk1/2-deficient MEFs was rescued by ectopic expression of WT ULK1 but not the KI mutant (Figure 3-5I), indicating that ULK1 catalytic activity is required for recruiting SEC24C to ERES. Whereas transient RNAi-mediated knockdown of Ulk1 or Sec $16 a$ in WT MEFs caused significant decreases $(P<0.001)$ in the number of SEC24C+ puncta, knockdown or knockout of Atg7, Atg13, or Atg14 did not (Figure 3-5J).

These results indicate that ULK/ATG1 has an evolutionarily conserved role in regulating ERES assembly, which is not shared by other autophagy-related proteins. ULK1 and ULK2 activities appear to converge on multiple aspects of SEC16A function, including the normal accumulation of SEC16A at ERES, and the assembly of SEC24Ccontaining ERES. These functions, however, appear to have different thresholds of sensitivity to loss of ULK/ATG1 function. SEC24C expression was relatively increased in hippocampal neurons, compared to the other SEC24 isoforms and neurons in other brain regions (data not shown). Therefore, these neurons may be particularly sensitive to the disrupted assembly of SEC24C-containing ERES, which occurs in the absence of $U l k 1 / 2$ expression.

\section{ULK1 and Related Kinases Regulate ER-to-Golgi Trafficking}

Because Ulk1/2-deficiency impaired the accumulation of SEC24C at ERES and only minimally affected VSVG trafficking (data not shown), which relies to a greater extent on the A and B isoforms of SEC24 than on the C and D isoforms for ER export (Bonnon et al., 2010), we examined the role of the ULKs in trafficking SEC24C-specific cargo. The serotonin transporter SERT interacts specifically with SEC24C, and its PM localization depends on SEC24C-dependent export from the ER (Montgomery et al., 2014; Sucic et al., 2011). SERT is expressed on the surface of platelets and specific types of neurons. It regulates the concentration of serotonin $(5-\mathrm{HT})$ at synapses and in blood plasma via uptake of 5-HT into neurons and platelets, respectively (Mercado and Kilic, 2010). Ulk1-ko MEFs showed reduced levels of the Endo H-resistant forms of SERTGFP in whole-cell extracts (Figure 3-6A, lower panels). Similarly, transient RNAimediated knockdown of Sec16a or Ulk1 in WT MEFs significantly reduced $(P<0.05$ and $P<0.01$, respectively) the ratio of Endo $\mathrm{H}$-resistant to Endo $\mathrm{H}-$ sensitive SERT-GFP (Figures 3-6B). However, knockdown of other autophagy-related genes (e.g., Atg7, Atg13, or Atg14) had no effect on ER-to-Golgi trafficking of SERT-GFP (Figures 3-6B). 
Figure 3-6. ULKs regulate ER-to-Golgi trafficking.

(A) Representative immunoblots of Biotin IPs (top panels) and GFP IPs (bottom panels) from SERT-GFP - transfected WT and Ulk1-ko MEFs. WT MEFs were incubated in the presence or absence of Endo H or peptide-N-glycosidase F (PNGase) to establish the migration pattern of the different glycosylated forms of SERT. (B) Mean ratios $( \pm$ SD) of Endo H-R SERT to total SERT in RNAi-treated samples from 2 independent experiments. ${ }^{*} P<0.01$ and ${ }^{\#} P<0.05$ and (ANOVA) when compared with Ctrl. (C) Mean percentages $( \pm$ SEM) of cells showing colocalization of AlexaFluor 647 -conjugated wheat germ agglutinin (WGA) and SERT-GFP. Data were acquired from 3 independent experiments, and more than 100 cells per population were scored. ${ }^{*} P<0.001$ (ANOVA) when compared with WT. (D) Representative merged pseudocolored images of SERTGFP - transfected WT and Ulk1-ko MEFs stained with AlexaFluor 647-conjugated wheat germ agglutinin (WGA) and DAPI. Scale bar: $10 \mu \mathrm{m}$. (E) Mean percentages $( \pm$ SEM) of cells with colocalized WGA and SERT-GFP. Data were acquired from 3 independent experiments, and more than 100 cells per population were scored in each experiment. ${ }^{*} P<0.002$ (ANOVA) when compared with empty vector-transduced cells. (F) Mean percentages $( \pm$ SEM) of siRNA transfected cells with colocalized WGA and SERT-GFP. Data were acquired from 3 independent experiments, and more than 100 cells per population were scored. $* P<0.001$ (ANOVA) when compared with siCtrltransfected cells. (G) Representative images of WT, unc-51 mutant and mod-5 mutant $C$. elegans stained with antibodies against MOD-5/SERT and 5-HT. The arrows highlight the 5-HT or MOD-5/SERT staining of NSM processes. 
A

UIk1: WT WT WT WT WT ko

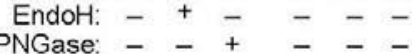

SERT-GFP $+\overline{+}+-\overline{+}+$

EndoH-R deGly SERT

EndoH-R

EndoH-S> deGly SERT

D WT MEFS

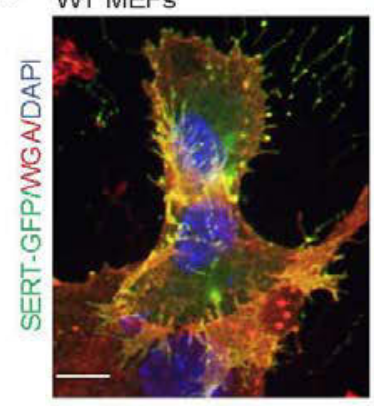

Ulk1 kO MEFs

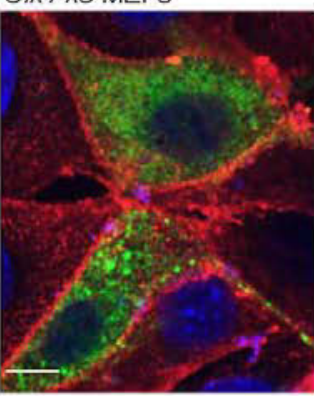

B

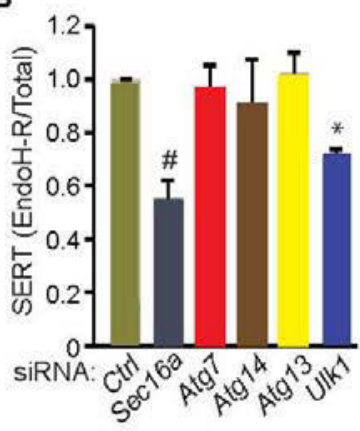

E

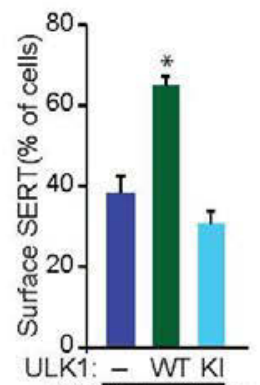

UIk1/2-def MEFs
C
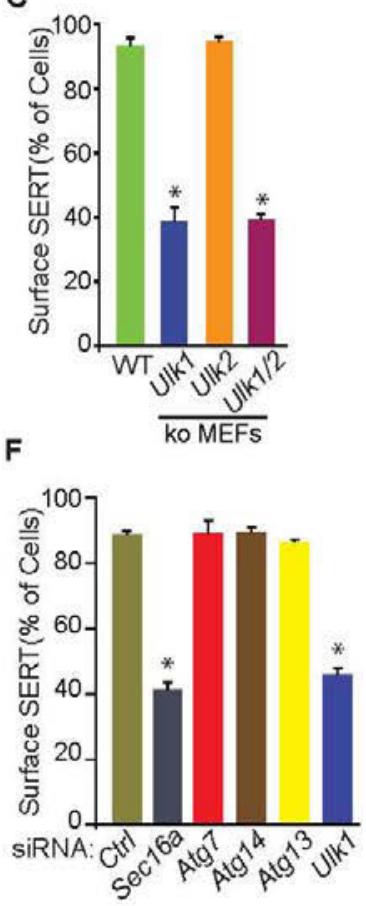

G
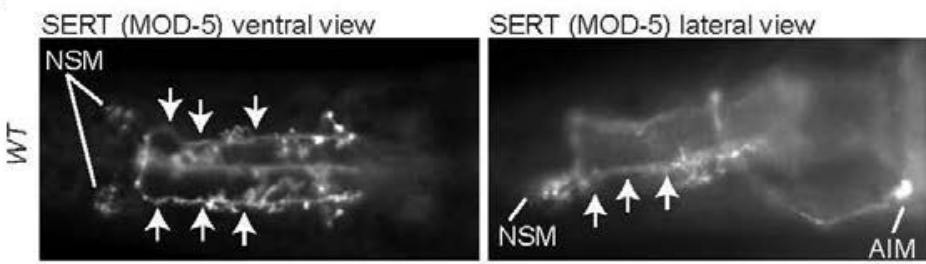

5-HT ventral view
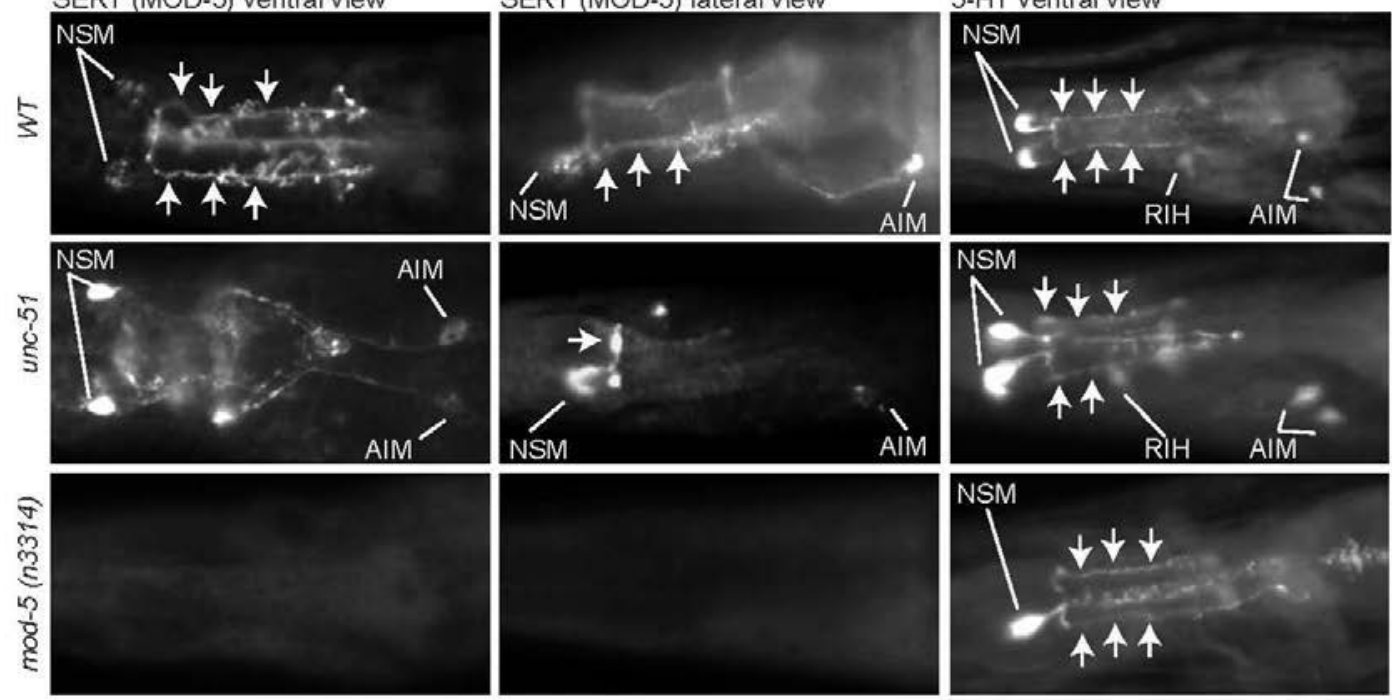
Treating cells with NHS-biotin prior to lysis allows selective biotinylation of surface proteins and provides a sensitive biochemical method of detecting defects in trafficking of proteins to the PM. Consistent with defective ER-to-Golgi trafficking of SERT, the levels of biotinylated surface SERT were reduced in anti-biotin IPs prepared from Ulk1ko MEFs (Figure 3-6A, upper panels). Ulkl-ko and Ulk1/2-dko MEFs also showed significant reduction $(P<0.001)$ in PM localization of SERT-GFP (Figure 3-6C, D). The defect in cell surface localization of SERT in Ulk1/2-deficient MEFs was rescued by expressing WT but not KI ULK1 (Figure 3-6E), indicating that ULK1 catalytic activity is required for SERT trafficking. Transient RNAi-mediated knockdown of Sec16a or Ulk1 significantly reduced $(\mathrm{P}<0.001)$ SERT localization to the PM of WT MEFs, but knockdown of other autophagy-related genes (e.g., $\operatorname{Atg} 7, \operatorname{Atg} 13$, or $\operatorname{Atg} 14)$ did not (Figure 3-6F).

In C. elegans, SERT (MOD-5) is expressed on the PM, along the length of axons of 5-HT-producing pharyngeal neurosecretory motor (NSM) neurons, with minimal localization of SERT/MOD-5 in cell bodies. Thus, $C$. elegans is a sensitive system for detecting defects in SERT trafficking in neurons in vivo. In contrast to its pattern of expression in WT NSM neurons, SERT/MOD-5 accumulated in the cell bodies of unc51-mutant NSM neurons and failed to localize to 5-HT-stained NSM axons (Figure 3-6G). These findings are consistent with a defect in ER-to-Golgi trafficking associated with disruption of UNC-51/ATG1 function. In addition to NSM neurons, AIM and RIH interneurons express SERT/MOD-5. These interneurons do not synthesize 5-HT; instead, they regulate 5-HT levels at extrasynaptic 5-HT targets via uptake mediated by SERT/MOD-5 (Jafari et al., 2011). As reported previously, SERT/MOD-5 is essential for accumulating 5-HT in AIM and RIH interneurons (Jafari et al., 2011), and these neurons in loss of function mod-5-mutant $C$. elegans showed no 5-HT accumulation (Figure 3-6G). However, the unc-51-mutant C. elegans showed normal levels of 5-HT accumulation (Figure 3-6G), suggesting that SERT trafficking and SERT-mediated 5-HT uptake is normal in AIM and RIH neurons. The basis for the cell-type specificity of ULK/ATG1-mediated SERT trafficking in C. elegans remains unknown. These findings indicate that ULK1 and homologs have an evolutionarily conserved and cell-type specific regulatory role in the trafficking of cargo such as SERT, which is not shared by other autophagy-related proteins, including ATG13.

\section{ULK-Mediated Phosphorylation of SEC16A at S846 Is Required for ER-to-Golgi Trafficking and ERES Assembly}

ULKs mediate SEC16A phosphorylation, and both proteins function in ER-toGolgi trafficking; thus, we investigated the role of ULK-mediated phosphorylation of SEC16A in this process. As mentioned above, we detected ULK1/2-dependent phosphorylation of SEC16A by using a $\mathrm{p}(\mathrm{S} / \mathrm{T})$ Phe antibody. Site-directed mutagenesis was used to substitute serine and threonine residues in SEC16A that matched the antibody-recognition motif with alanines. The resulting GFP-SEC16A mutants were screened for their ability to disrupt ULK1-dependent phosphorylation (data not shown). We observed a reproducible decrease in the ULK1-dependent phosphorylation signal on 
the GFP-SEC16A S846A-mutant protein compared to WT (data not shown). To confirm that S846 was phosphorylated in an ULK1-dependent manner, we reintroduced the S846A mutation into a MYC-DDK-tagged SEC16A-expression construct. Use of this construct demonstrated that the S846A substitution was sufficient to abolish the ULK1dependent phosphorylation signal on SEC16A that was recognized by the $\mathrm{p}(\mathrm{S} / \mathrm{T}) \mathrm{Phe}$ antibody (Figure 3-7A).

To determine the consequence of ULK-mediated phosphorylation of SEC16A at S846, we expressed WT SEC16A, the phospho-defective S846A mutant, and the phosphomimetic S846D mutant in Sec16a-depleted cells and assessed their ability to restore the defects in ERES assembly and ER-to-Golgi trafficking common to Ulk1/2deficient and Sec16a-depleted MEFs. First, we detected fewer SEC16A ${ }^{+}$puncta in Sec 16a-depleted cells upon overexpression of the S846A mutant than in cells expressing WT SEC16A or the S846D mutant, despite comparable levels of protein (Figure 3-7B, C). The number of SEC $24 \mathrm{C}^{+}$puncta was also fewer in Sec16a-depleted cells expressing the S846A mutant than in those expressing WT SEC16A or the S846D mutant (Figure 3-7B, D). These results are consistent with the notion that ULK-mediated phosphorylation of SEC16A is required for efficient recruitment and/or retention of SEC16A and SEC24C at ERES. WT SEC16A did not increase the number of SEC24C ${ }^{+}$ puncta in Ulk1-deficient MEFs (Figure 3-7E, F), suggesting that the residual Ulk2 expression in these MEFs was insufficient to yield high enough levels of phosphorylated SEC16A to promote accumulation of SEC24C at ERES. In contrast, expressing the S846D mutant in Ulk1-deficient MEFs significantly increased $(P<0.001)$ the number of $\mathrm{SEC}_{16 \mathrm{~A}^{+}}$puncta and SEC $24 \mathrm{C}^{+}$puncta and the proportion of puncta showing colocalization of SEC16A and SEC24C (Figure 3-7E, H). Similarly, the defect in SERT trafficking in Ulk1-ko MEFs was rescued by overexpressing the S846D SEC16A mutant but not the WT SEC16A or the S846A mutant (Figure 3-7I, J). These data indicate that ULK1/2-mediated phosphorylation of SEC16A at S846 drives the assembly of COPII components at ERES and trafficking of associated secretory cargo.

\section{The Defect in ER-to-Golgi Trafficking in ULK-Deficient Cells Leads to an ER- Stress Response That Is Reversed by the S846D SEC16A Mutant}

Defects in ER-to-Golgi trafficking can cause proteins to accumulate in the ER and activate the UPR system, but we did not detect any evidence of UPR activation in Ulk1and/or Ulk2-ko MEFs under basal growth conditions (data not shown) or in Ulk1-ko MEFs expressing VSVG-GFP (Figure 3-8A). By contrast, Ulk1-ko MEFs expressing SERT-GFP showed a significant increase $(P<0.001)$ in nuclear localization of CHOP (Figure 3-8A, B). Moreover, expression of the S846D SEC16A mutant in Ulk1-ko MEFs, which rescued the defect in ER-to-Golgi trafficking of SERT, prevented nuclear localization of CHOP (Figure 3-8C, D). These findings indicate that the defect in ULKand SEC16A-dependent ER-to-Golgi trafficking of certain cargo activates the UPR pathway, similar to that observed in Ulk1/2-deficient hippocampal neurons." 
Figure 3-7. ULK-mediated phosphorylation of SEC16A at S846 is required for assembly of SEC24C ${ }^{+}$ERES and ER-to-Golgi trafficking.

(A) Representative immunoblots of DDK IPs prepared from $293 \mathrm{~T}$ cells transfected with either MYC-DDK-tagged WT SEC16A or a mutant form of MYC-DDK-tagged SEC16A harboring the S846A and the specified ULK1-expression construct. (B) Representative pseudocolored images of MEFs stained with DAPI and antibodies against MYC-DDK-tagged SEC16A and SEC24C. SA: SEC16A S846A mutant; SD: SEC16A S846D mutant. (C) Mean numbers $\left( \pm\right.$ SEM) of SEC16A ${ }^{+}$puncta per cell. (D) Mean numbers $\left( \pm\right.$ SEM) of SEC $24 C^{+}$puncta per cell . Ten cells $\left(\mathrm{DDK}^{+}\right.$, if appropriate) per population were scored in $\mathbf{C}$ and $\mathbf{D}$. $* P<0.001$ (ANOVA). (E) Representative pseudocolored images of MEFs stained with DAPI and antibodies against SEC24C and MYC-DDK-tagged SEC16A. (F) Mean numbers ( \pm SEM) of SEC24C ${ }^{+}$puncta per cell. (G) Mean numbers $\left( \pm\right.$ SEM) of SEC16 ${ }^{+}$puncta per cell. (H) Mean percentages $( \pm$SEM) of SEC16A ${ }^{+}$puncta that were also SEC24C ${ }^{+}$. Ten cells (DDK ${ }^{+}$, if appropriate) per population were scored in $\mathbf{F}-\mathbf{H}$. Scale bar $=10 \mu \mathrm{m}$. ${ }^{*} P<0.001$ and ${ }^{\#} P<0.05$ when compared with WT (ANOVA). (I) Representative merged pseudocolored images of MEFs stained with AlexaFluor 647-conjugated WGA and antibodies against MYCDDK-tagged SEC16A. White arrowheads indicate PM. (J) Mean percentages $( \pm$ SEM) of cells from each population with colocalized WGA and GFP. Data were acquired from 3 independent experiments, and more than 100 cells per population were scored in each experiment. Scale bar: $10 \mu \mathrm{m} .{ }^{*} P<0.001$ (ANOVA) when compared with empty vector. 


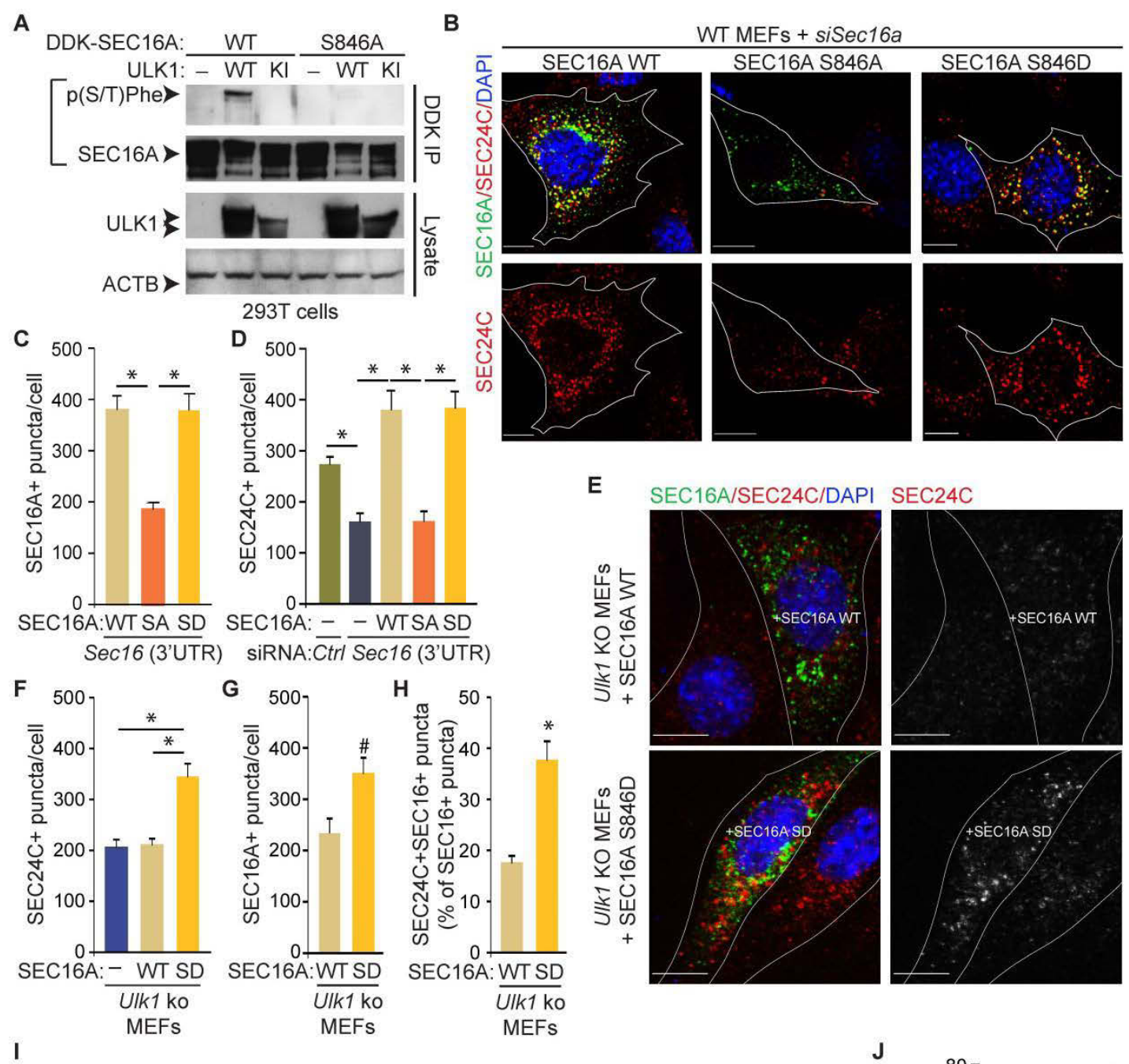

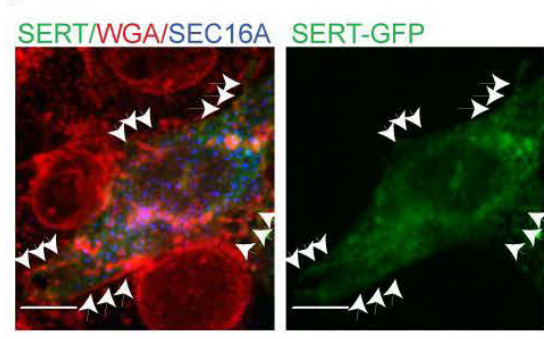

Ulk1 ko MEFs

+ SEC16A WT

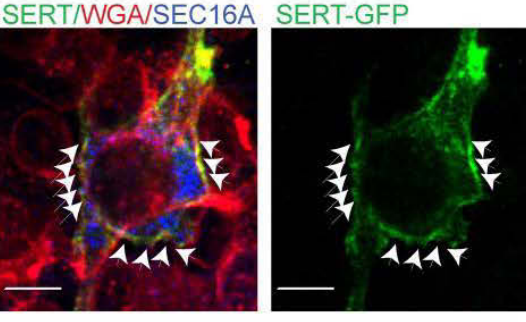

Ulk1 ko MEFs

+ SEC16A SD

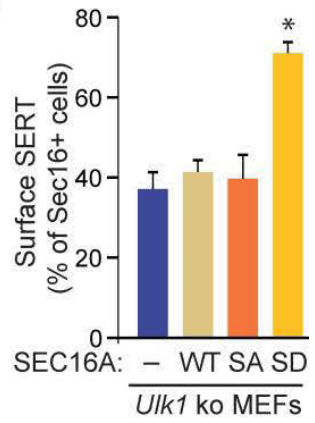



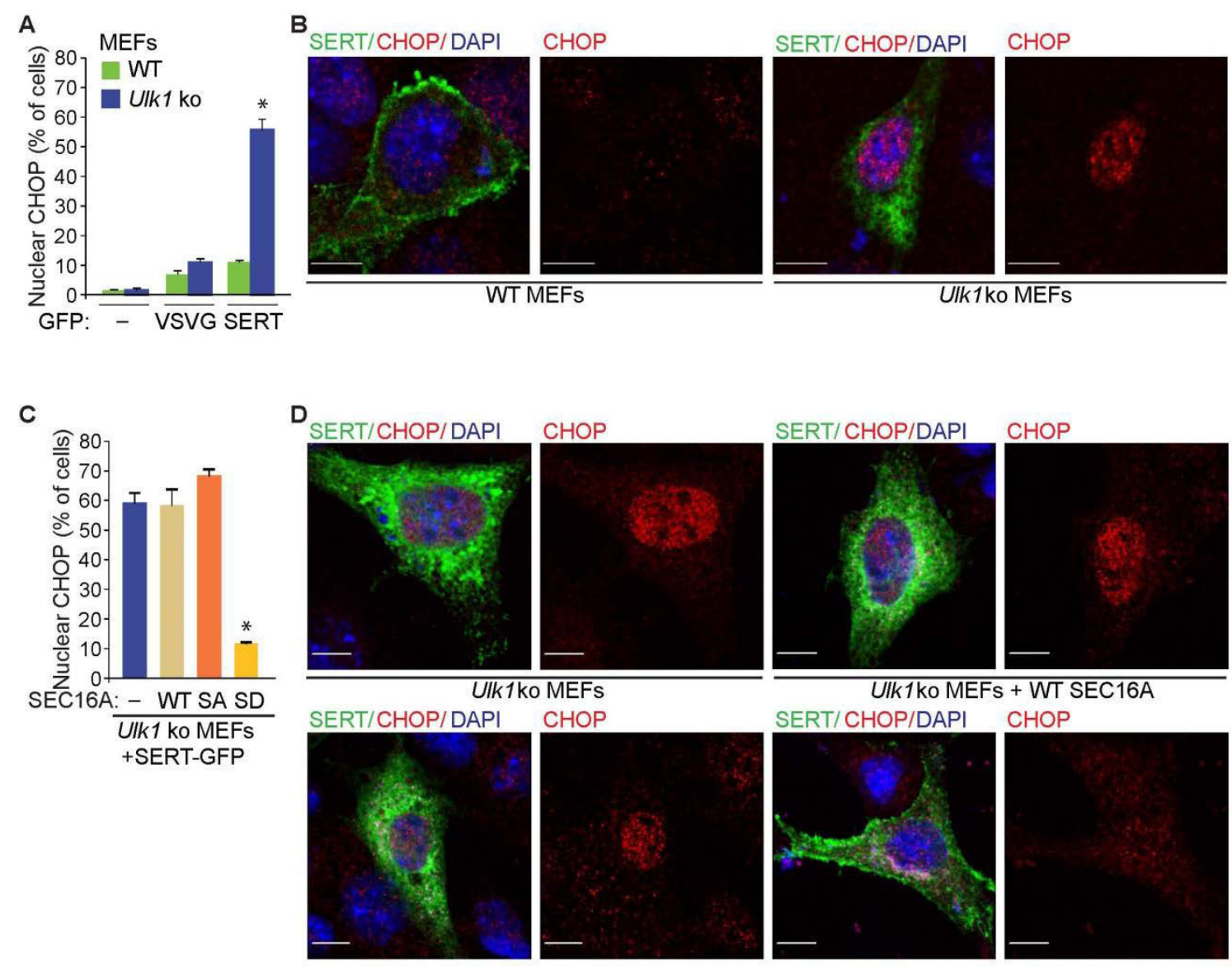

Ulk1ko MEFs + SEC16A SA mutant

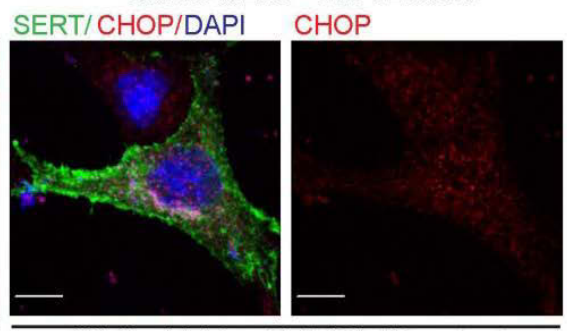

Ulk1 ko MEFs + SEC16A SD mutant

Figure 3-8. SEC16A mutant with the phosphomimetic S846D overcomes the defect in ER-to-Golgi trafficking and prevents activation of the UPR in ULKdeficient cells.

(A) Mean percentages $( \pm$ SEM) of cells with nuclear staining of CHOP. Data were acquired from 3 independent experiments, and more than $100 \mathrm{GFP}^{+}$cells per population were scored in each experiment. ${ }^{*} P<0.001$ (ANOVA) when compared with empty vector-transduced cells. (B) Representative pseudocolored images of WT and Ulkl-ko MEFs transfected with indicated GFP-tagged cargo [i.e, (-), empty vector; VSVG-GFP or SERT-GFP] stained with an antibody against CHOP and DAPI. Scale bar: $10 \mu \mathrm{m}$. (C) Mean percentages $( \pm \mathrm{SEM})$ of cells with nuclear staining of $\mathrm{CHOP}$. Data were acquired from 3 independent experiments, and more than $100 \mathrm{GFP}^{+} \mathrm{DDK}^{+}$cells per population were scored in each experiment. $* P<0.001$ (ANOVA) when compared with empty vector- transduced cells. SA: S846A SEC16A mutant; SD, S846D SEC16A mutant. (D) Representative pseudocolored images of MEFs stained with antibodies against MYCDDK-tagged SEC16A (channel not shown) and CHOP and DAPI. Scale bar: $10 \mu \mathrm{m}$. 


\section{CHAPTER 4. AUTOPHAGY-INDEPENDENT ROLE OF ULK1 AND ULK2 IS ESSENTIAL FOR AXON GUIDANCE IN THE DEVELOPING MOUSE FOREBRAIN}

\section{Ulk1/2-Deficiency in the CNS Results in Abnormal Axon Guidance}

Because of the observation that approximately $40 \%$ of CNS specific Ulk1/2-cdko mice die shortly after birth and the previously described role of ULK/ATG1 homologues in axon guidance and growth, we decided to examine the integrity of the major axon tracts formed by projection neurons in Ulk1/2-DKO mice. Towards this end, we interbred $U l k 1^{+-} ; U l k 2^{-/-}$mice. Twice the number of $U l k 1^{+/+} ; U l k 2^{-/-}(63 / 206 ; 30.58 \%)$ compared to $U l k 1^{-/} ; U l k 2^{-/}(29 / 206 ; 14.08 \%)$ embryos were identified upon genotyping at E18.5, suggesting that loss of both Ulk1 and Ulk2 increased embryonic mortality. Nevertheless, we observed no significant difference in body weight (Figure 4-1A) or gross brain morphology (Figure 4-1B) in E18.5 Ulk1/2-DKO embryos compared to littermate controls $\left(U l k 1^{+/+} U l k 2^{-/-}\right.$or $\left.U l k 1^{+/-} U l k 2^{-/-}\right)$. Visual inspection of newborn pups and genotyping of pups on or after post-natal day 1 (P1) confirmed the previously reported perinatal mortality of live born Ulk1/2-DKO pups (Cheong et al., 2011; Cheong et al., 2014; Kundu et al., 2008) .

Commissural projection neurons project their axons to the contralateral cortex, thus connecting the two cortical hemispheres. The cell bodies of commissural projection neurons in rodents reside primarily in cortical layers II/III (80\%), layer V (20\%) and to a lesser extent layer VI; their axons traverse the telencephalic midline, giving rise to three commissural tracts: the corpus callosum $(\mathrm{CC})$, the hippocampal commissure $(\mathrm{HC})$ and the anterior commissure $(\mathrm{AC})$. We used an antibody against the axonal marker L1 cell adhesion molecule (L1) to examine the three commissural tracts in E18.5 embryos. The CC was thinner in Ulk1/2-DKO embryos compared to that in littermate controls (Figure 4-1C) and many of the callosal axons were overfasciculated (Figure 4-1D1, D1', D4, D4'). Although the AC in Ulk1/2-DKO embryos was severely hypoplastic, the HC showed no overt abnormalities (Figure 4-1D2, D2', D5, D5').

We also examined the integrity of the corticothalamic axons (CTAs), which originate from neurons residing in layer VI of the cortex and project to the thalamus, and thalamocortical axons (TCAs), which originate from neurons in various thalamic nuclei and project to corresponding cortical areas. The CTAs and TCAs of Ulk1/2 dko mice showed several abnormalities as they crossed the pallial-subpallial boundary (PSPB). Instead of forming a parallel fan-like structure, the Ulk1/2-deficient axons were disorganized, overfasciculated and traversed the PSPB more anteroventrally than axons in littermate controls (Figure 4-1D3, D3', D6, D6', E). The abnormal path of the CTAs and TCAs in Ulk1/2-deficient animals was already evident at E14.5, around the time when the CTAs and TCAs first begin crossing the PSPB (Figure 4-1F-G), suggesting that ULK1 and ULK2 regulate an intermediate step in axon pathfinding (i.e. crossing midline or specific boundaries). 
Figure 4-1. ULK1 and ULK2 are required for axonal guidance in the forebrain. (A) No significant difference in body weight of control $\left(U l k 1^{+/+} ; U l k 2^{-/-}\right.$and $U l k 1^{+/-} ; U l k 2^{-/-}$ ) and DKO mice at E18.5. (B) No overt differences in gross brain morphology between control and DKO mice at E18.5. (C) Quantification of the dorsoventral width of the CC of control $(\mathrm{n}=5)$ and $U l k 1 / 2$-DKO mice $(\mathrm{n}=5)$ at E18.5. The average $\mathrm{CC}$ width of each mutant was normalized to that of its corresponding control. ** $P<0.01$. (D) Brain sections of E18.5 control (D1, D2, D3) and $U l k 1 / 2-\mathrm{DKO}(\mathbf{D 4}, \mathbf{D 5}, \mathbf{D 6})$ were stained with antibodies against the general axonal marker L1 (pseudocolored green). The callosal axons are heavily overfasciculated, and the corpus callosum is thinner in Ulk1/2-DKO mice $\left(\mathbf{D 1}^{\prime}, \mathbf{D 4}^{\prime}\right)$. The anterior commissure fails to develop $\left(\mathbf{D 2}^{\prime}, \mathbf{D 5}^{\prime}\right)$. CTAs and TCAs progressed abnormally as they cross the PSPB $\left(\mathbf{D 3}^{\prime}, \mathbf{D 6}^{\prime}\right)$. The $U l k 1 / 2$-DKO axons are highly disorganized and extend aberrantly towards the external capsule (EC) (arrows in D5-D6). The CTAs and TCAs are also overfasciculated. (E) Sagittal view of the the abnormal CTA and TCA tracts highlighted by arrowheads. (F) The axon pathfinding defects in Ulk1/2-DKO mice are detected as early as E14.5 (dashed lines), and (G) number of axons crossing PSPB is quantified from control $(\mathrm{n}=3)$ and $U l k 1 / 2$-DKO mice $(\mathrm{n}=3)$. Abbreviations: AC, anterior commissure; CC, corpus callosum; dTh: dorsal thalamus; St, striatum. Scale bars: $500 \mu \mathrm{m}$ in D1-D6, and E, G; $250 \mu \mathrm{m}$ in D1'-D6'. 


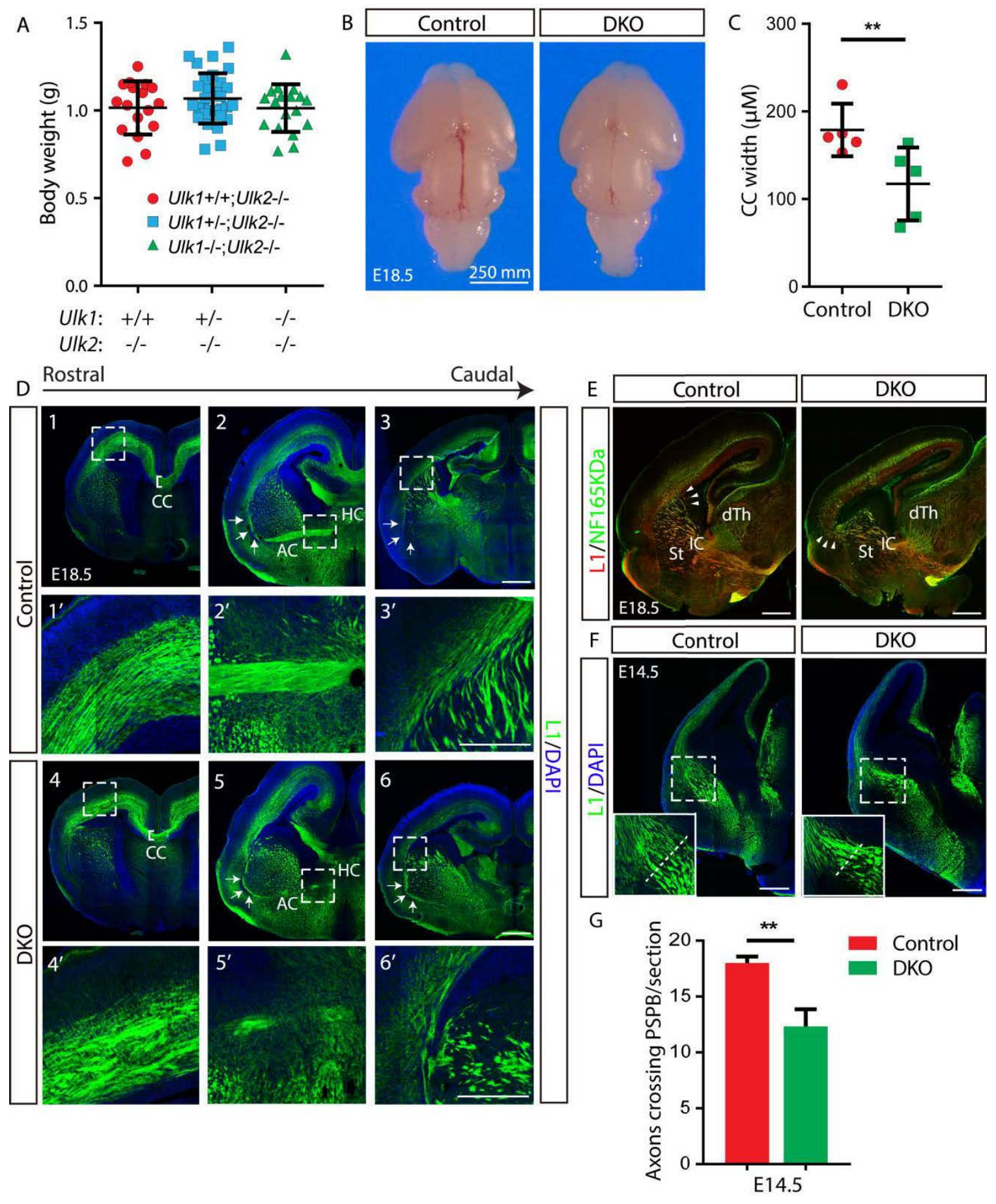




\section{ULK1 and ULK2 Regulate Axon Guidance via an Autophagy-Independent Pathway}

FIP200 and ATG13 are components of the canonical 3-MDa ULK/ATG1 kinase complex that regulates both selective and non-selective autophagy in response to cellular stress (Alers et al., 2012; Mizushima, 2010). ULK/ATG1 also interacts with the COPII scaffold SEC16A to promote ER-to-Golgi trafficking of specific cargo in the absence of stress (Joo et al., 2016). Therefore, we immunoprecipitated endogenous ULK1 from extracts prepared from the cerebral cortex of newborn (P0) mice to determine which of these interactions may be relevant in the developing cortex. While we were able to detect the interaction between ULK1, ATG13 and FIP200 in both P0 and adult cortex, the interaction between ULK1 and SEC16A was restricted to P0 cortex (Figure 4-2A), raising the possibility that either the autophagy-inducing function of ULK/ATG1 or its role in ER-to-Golgi trafficking may play an important role in the regulation of axon guidance.

In order to assess the contribution of the autophagy-inducing function of ULK/ATG1 in axon guidance, we decided to investigate the extent of overlap in the neurodevelopmental phenotypes of mice lacking CNS expression of Fip200, Atg7 and

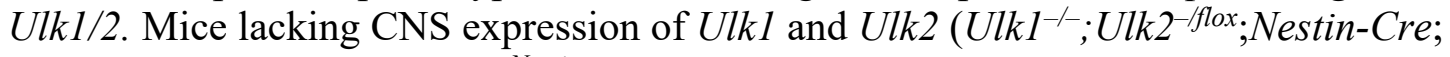
hereafter referred to as $U l k 1 / 2^{\text {Nestin }}$ cDKO mice) show progressive hippocampal degeneration beginning around 8 weeks of age (Joo et al., 2016). Although some of the Ulk1/2 $2^{\text {Nestin }} \mathrm{cDKO}$ mice survived up to 4 months, $40 \%$ of them died within the first 24 hours after birth (Joo et al., 2016). The surviving mice showed no accumulation of P62+ or Ubiquitin+ deposits in all the cortical layer either (Figure 4-2B) or any appreciable increase in steady state levels of P62 (Joo et al., 2016). After confirming the loss of Ulk1 and Ulk2 expression in the brains of $U l k 1 / 2^{\text {Nestin }} \mathrm{cDKO}$ mice by RT-qPCR analyses (Figure 4-3A), we examined L1 staining of brain sections from $U l k 1 / 2^{\text {Nestin }} \mathrm{cDKO}$ mice and littermate controls on post-natal day P1. These stains revealed dysgenesis of the corpus callosum (including the presence of Probst bundles), hypoplastic AC, and disruption of CTAs and TCAs in $U l k 1 / 2^{\text {Nestin }} \mathrm{cDKO}$ mice (Figure 4-2B), similar to that observed in the Ulk1/2-DKO mice. Mice lacking CNS expression of Fip200 (Fip200 Nestin $\mathrm{cKO}$ ) or $\operatorname{Atg} 7$ ( $\operatorname{Atg} 7^{\text {Nestin }} \mathrm{cKO}$ ) showed the expected accumulation of P62+ and ubiquitin+ deposits in all the cortical layers (layer I-VI) examined (Figure 4-2B), but no defects in commissural axon tracts or disruption of CTAs and TCAs (Figure 4-3B).

We also examined the trajectory of unmyelinated axon projections (i.e. mossy fibers) from granule cells in the dentate gyrus to the hippocampal CA3 regions in the brains of Ulk1/2 $2^{\text {Nestin }} \mathrm{cDKO}$, Fip200 $0^{\text {Nestin }} \mathrm{cKO}$, Atg $7^{\text {Nestin }} \mathrm{cKO}$ and littermate controls at $\mathrm{P} 21$. Immunostaining with antibodies against the neuronal marker $\mathrm{NeuN}$ and Neurofilament 165 allowed visualization of the mossy fibers located above (i.e., suprapyramidal bundle) and below (i.e., infrapyramidal bundle) the pyramidal cell layer of the CA3 (Figure 4-4A1). In Atg $7^{\text {Nestin }}$ cDKO, Fip200 ${ }^{\text {Nestin }}$ cDKO mice and control animals, the fibers in the infrapyramidal bundle extended along the pyramidal cell layer in the proximal portion of CA3 (i.e closer to the dentate gyrus), and then crossed the pyramidal cell layer at mid and distal portions of CA3 to join the mossy fibers in the suprapyramidal bundle (Figure 4-4A4, A5). Mossy fibers in the $U l k 1 / 2^{\text {Nestin }} \mathrm{cDKO}$ mice 
A
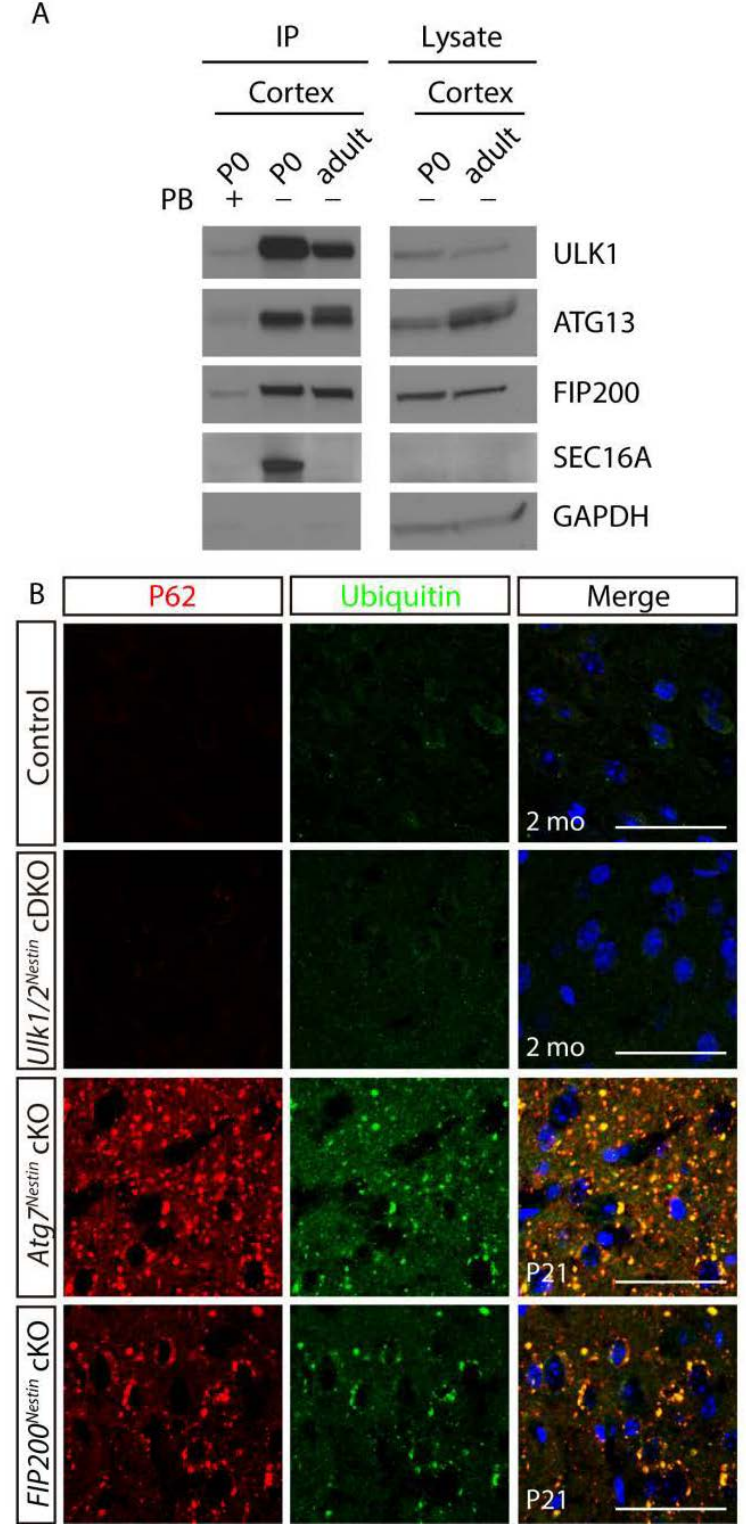

Figure 4-2. ULK1 and ULK2 are not required for autophagy-mediated turnover of ubiquitinated protein in the cerebral cortex.

(A) Immunoprecipitation (IP) of ULK1 using extracts prepared from the cortex of P0 and adult WT mice and performed in the presence or absence of epitope-specific blocking peptides (PB). The interaction between ULK1, ATG13 and FIP200 was detected in extracts prepared from $\mathrm{P} 0$ and adult cortex; whereas the interaction between ULK1 and SEC16A was only detected in extracts prepared from P0 (but not adult) cortex. (B) Immunostains performed on sections of cortex from control, Ulk1/2Nestin $\mathrm{cDKO}, \mathrm{Atg} 7^{\text {Nestin }}$ cKO and FIP200 Nestin $\mathrm{cKO}$ mice using antibodies against P62 (pseudocolored red) and Ubiquitin (pseudocolored green). Sections were counterstained with DAPI (pseudocolored blue). Scale bars: $50 \mu \mathrm{m}$. 

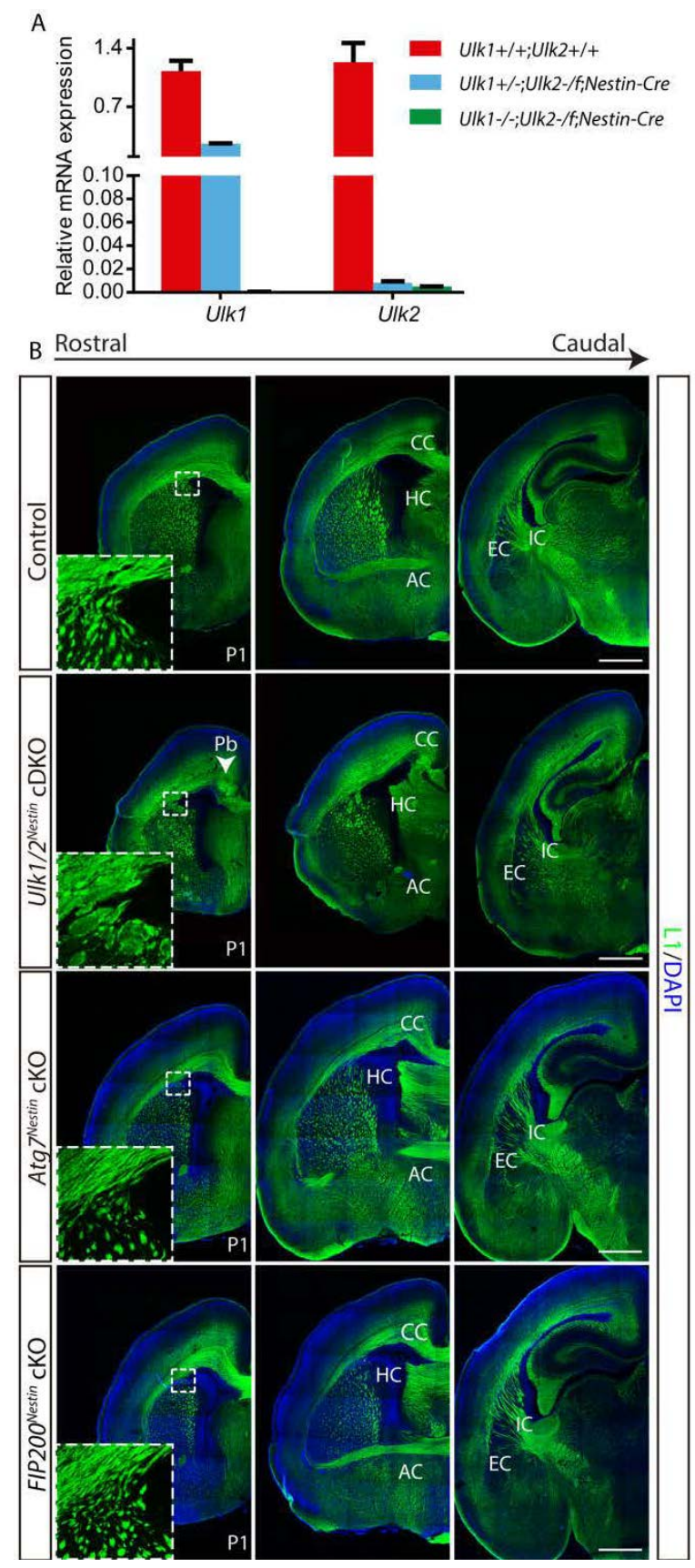

Figure 4-3. FIP200 and ATG7 are not required for axon guidance in the forebrain.

(A) Real-time PCR confirms the loss of both Ulk1 and Ulk2 in the brain of $U l k 1 / 2^{\text {Nestin }}$ cDKO mice. (B) L1 staining of serial sections of $U l k 1 / 2^{\text {Nestin }} \mathrm{cDKO}$ brains at P1 reveals abnormally fasciculated axons, including in the CTAs and TCAs (inset), corpus callosum dysgenesis and anterior commissure hypoplasia. These axon guidance abnormalities are similar to those identified in the Ulk1/2-DKO mice and are not observed in autophagydeficient $A \operatorname{tg} 7^{\text {Nestin }} \mathrm{cKO}$ or Fip200 ${ }^{\text {Nestin }} \mathrm{cKO}$ mice. Abbreviations: AC, anterior commissure; CC, corpus callosum; IC, internal capsule; EC, external capsule; HC, hippocampal commissure; Pb, Probst bundle. Scale bars: $500 \mu \mathrm{m}$ 

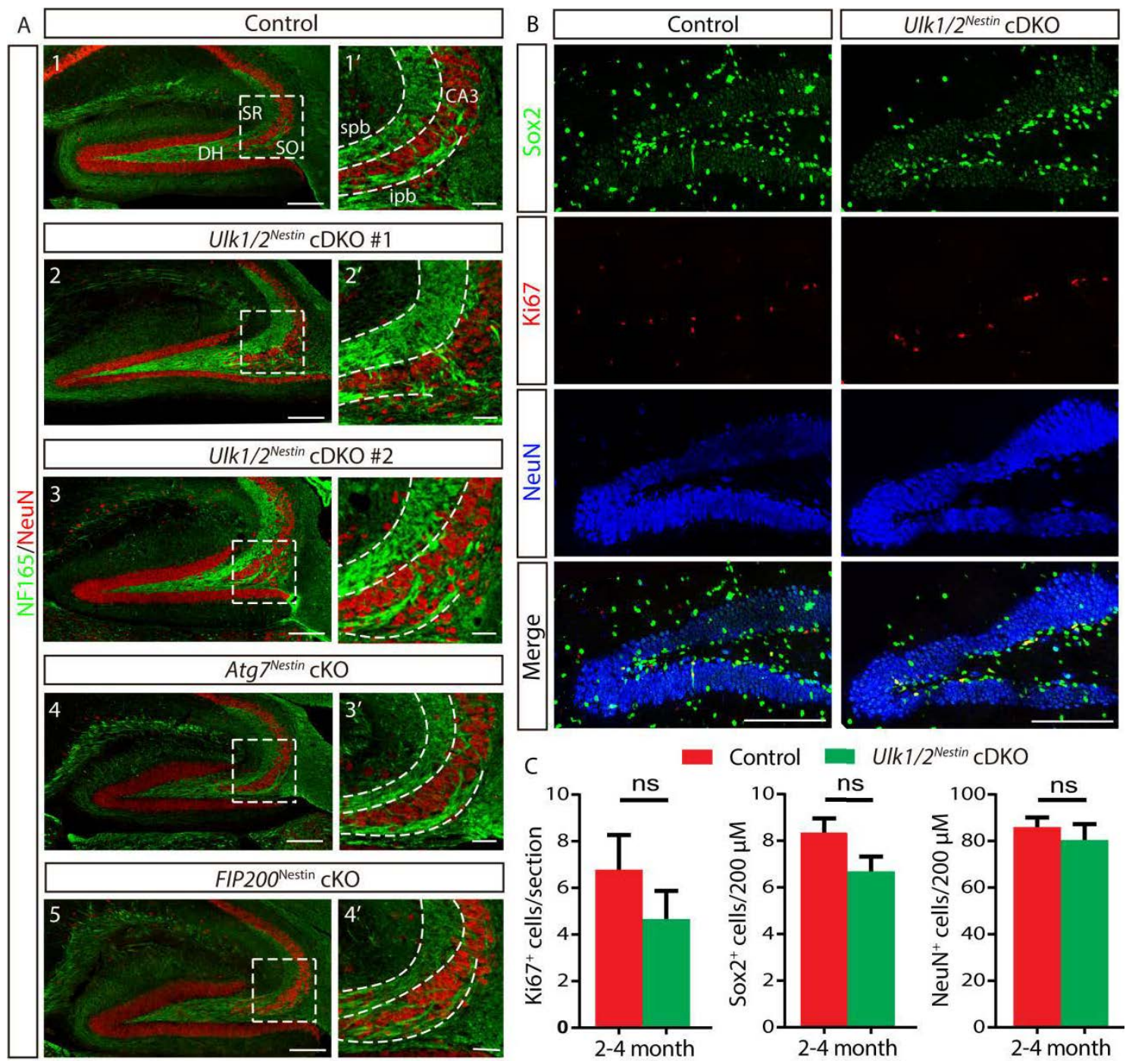

Figure 4-4. ULK1 and ULK2 regulate mossy fiber guidance via an autophagy independent-mechanism.

(A) Sections of hippocampus from adult control, Ulk1/2 Nestin $\mathrm{cDKO}$ mice, Atg $7^{\text {Nestin }} \mathrm{cKO}$, and FIP200 Nestin $\mathrm{cKO}$ mice were immunostained with antibodies against NF165 and NeuN. Representative images show the abnormal projections from the infrapyramidal bundle in two Ulk1/2 $2^{\text {Nestin }}$ cDKO mice (A2', A3') but not in the $\operatorname{Atg}^{\text {Nestin }}$ cKO (A4') or FIP200 Nestin $\mathrm{cKO}$ mice (A5'). (B) Immunostaining of markers for mitotic cells (Ki67), neuronal stem cells (Sox2), and mature neurons (NeuN) in the dentate gyrus of adult control and Ulk1/2 $2^{\text {Nestin }}$ cDKO mice. (C) The mean number $\pm \mathrm{SEM}$ of $\mathrm{Ki}^{+} 7^{+}$, Sox $2^{+}$, and $\mathrm{NeuN}^{+}$cells in the dentate gyrus showed no statistical significance between controls $(\mathrm{n}=$ 3) and $U l k 1 / 2^{\text {Nestin }} \mathrm{cDKO}(\mathrm{n}=3)$ mice. Abbreviations: DH: dendate hilus; ipb:

infrapyramidal bundle of mossy fibers; SO: stratum oriens; spb: suprapyramidal bundle of mossy fibers; SR: stratum radiatum; ns: not significant. Scale bars: $200 \mu \mathrm{m}$ in A1-A5, $\mathbf{B} ; 50 \mu \mathrm{m}$ in $\mathbf{A 1} \mathbf{1}^{\prime}-\mathbf{A 5}$ '. 
entered the proximal part of the infrapyramidal region within CA3 and either terminated prematurely, turned prematurely into or aberrantly away from the pyramidal cell layer (Figure 4-4A2, A3). Together, these results suggest that the role of ULK1 and ULK2 in axonal guidance occurs via an autophagy-independent pathway.

FIP200 is essential for maintenance of the neuronal stem cell pools in post-natal brains (Wang et al., 2013), and the dentate gyrus is one of only two brain regions with continuous neurogenesis in adult animals. Therefore, we examined the dentate gyrus of Ulk1/2 $2^{\text {Nestin }}$ cDKO mice in more detail, but observed no obvious difference in the number of neuronal stem cells, mitotic neurons, or mature neurons in the dentate gyrus in 2-4 month old animals (Figure 4-4B, C). Thus, consistent with the notion that the ULKs are dispensable for autophagy in the central nervous system in unstressed animals, the ULKs are not required for the FIP200- and autophagy-mediated post-natal regulation of neural stem cell function.

\section{ULK1 and ULK2 Are Not Essential for Cortical Neurogenesis}

We next sought to further characterize the neurodevelopmental defects in $U l k 1 / 2$ deficient mice in order to gain some insight into non-canonical functions of the mammalian ATG1 homologues. In order to exclude the possibility that the abnormalities in the callosal axons, CTAs and TCAs in Ulk1/2-deficient mice were caused by impaired production of cortical projection neurons, we examined cortical layer structure of E18.5 DKO brains. Nissl staining and the expression of the callosal neuron marker Satb2, Layer V marker Ctip2, and Layer VI marker Tbr1 were all normal in the DKO cortex (Figure 4-5A, B). Thus, ULK1 and ULK2 appear to be dispensable for specification of projection neurons in the embryonic cortex. The Ulk1/2-DKO embryos did not show an increase in apoptosis (Figure 4-5C, D) or neural tube closure defects (data not shown), both of which are seen in Ambral-deficient animals (Fimia et al., 2007).

\section{ULK1 and ULK2 Regulate the Midline Crossing of Callosal Axons}

In order to trace the projection of callosal axons across the midline, we embedded Dil crystals into the parietal cortex of control and Ulk1/2-DKO embryos at E18.5. Compared to that of controls, the fluorescence intensity of callosal axons reaching the contralateral cortex was dramatically reduced in the $U l k 1 / 2-\mathrm{DKO}$ brains (Figure 4-6A). Luxol Fast Blue staining for myelinated axons also showed significantly reduced CC thickness in Ulk1/2 Nestin $\mathrm{cDKO}$ brains at 2 months, indicating persistent deformity of the $\mathrm{CC}$ in Ulk1/2-deficient mice (Figure 4-6B, C).

Abnormal CC development can result from cell autonomous defects, or various cell non-autonomous abnormalities, such as environmental/axon guidance cues from midline glia and guidepost neurons. Having ruled out obvious defects in cortical neurogenesis (Figure 4-5), we examined the midline glial structures and guidepost neuron population. Glial cells stained with GFAP were properly formed in the midline 

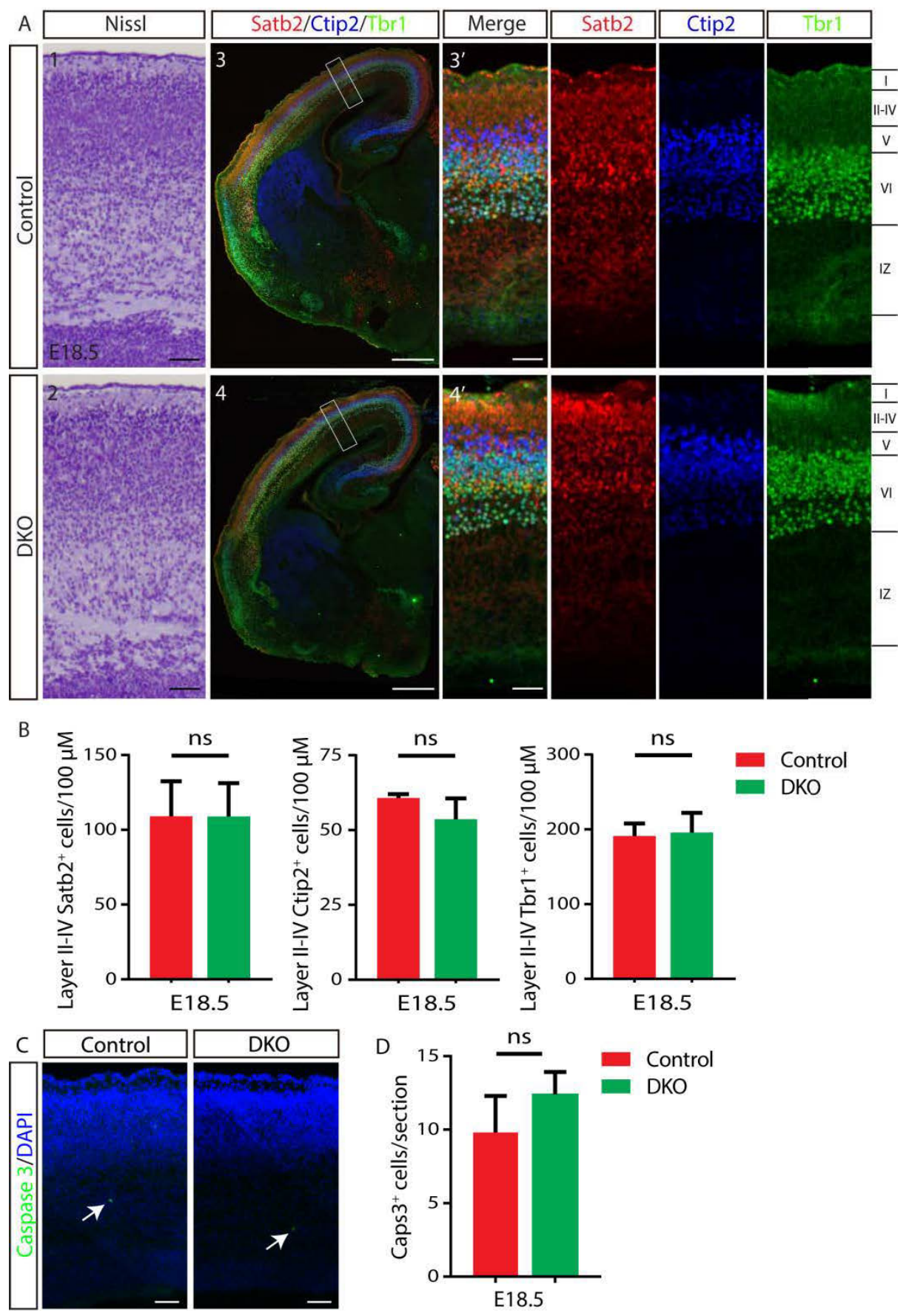

Figure 4-5. ULK1 and ULK2 are not essential for neurogenesis.

(A) Niss1 staining (A1-A2) and expression of the callosal neuron marker Satb2, layer V marker Ctip2, and layer VI marker Tbr1 in control and Ulk1/2-DKO cortices at E18.5 (A3-A4), and the quantifications of Satb2 ${ }^{+}$, Ctip2 ${ }^{+}$, and $\mathrm{Tbr}^{+}$cells in layer II-IV, layer $\mathrm{V}$, and layer VI, respectively, are statistically not significant (ns), indicating that $U l k 1 / 2$ are not required for neurogenesis. (C-D) Staining of cleaved Caspase 3 on cortices from control and $U l k 1 / 2-\mathrm{DKO}$ at E18.5, and number of Caspase $3+$ cells per section is quantified ( $\mathrm{n}=3$ for both control and $U l k 1 / 2-\mathrm{DKO})$. Scale bars: $50 \mu \mathrm{m}$ in A1-A2, A3'A4', and $\mathbf{C}$; $500 \mu \mathrm{m}$ in $\mathbf{A 3}-\mathbf{A 4}$. 
Figure 4-6. ULK1 and ULK2 regulate development of the corpus callosum. (A) Dil was placed in the parietal cortex of E18.5 Ulk1/2-DKO $(\mathrm{n}=2)$ and control $(\mathrm{n}=2)$ mice to trace the midline crossing of callosal axons. Representative sections on the left and bar graph on the left highlight the dramatic reduction in fluorescence intensity in the contralateral cortex (rostral and caudal levels) of Ulk1/2-DKO mice (asterisks). (B) Luxol Fast Blue and Nissl staining reveals persistent anomalies of the corpus callosum (CC) in the adult $U l k 1 / 2^{\text {Nestin }}$ cDKO mice. (C) The dorsoventral width of the $\mathrm{CC}$ of adult control $(\mathrm{n}=3)$ and $U l k 1 / 2^{\text {Nestin }}$ cDKO mice $(\mathrm{n}=3)$ was quantified, and the average width of each mutant was normalized to that of its corresponding control. There is a significant $(* *$ $P<0.01)$ reduction in the width of the corpus callosum in $U l k 1 / 2^{\text {Nestin }} \mathrm{cDKO}$ mice relative to that in controls. (D) Representative brain sections from E18.5 Ulk1/2-DKO and P0 $U l k 1 / 2^{\text {Nestin }} \mathrm{cDKO}$ mice and age-matched controls. The midline glial structure developed properly. (E) Calretinin immunostaining of the glutamatergic guidepost neurons in control and Ulk1/2-DKO brains at E15.5 and E18.5 showed that the production of those neurons was not altered in the $U l k 1 / 2$-deficient mice. Scale bars: $500 \mu \mathrm{m}$ in A1-A4, and B1-B2; $100 \mu \mathrm{m}$ in A1'-A4', B1'-B2', and D-E. 

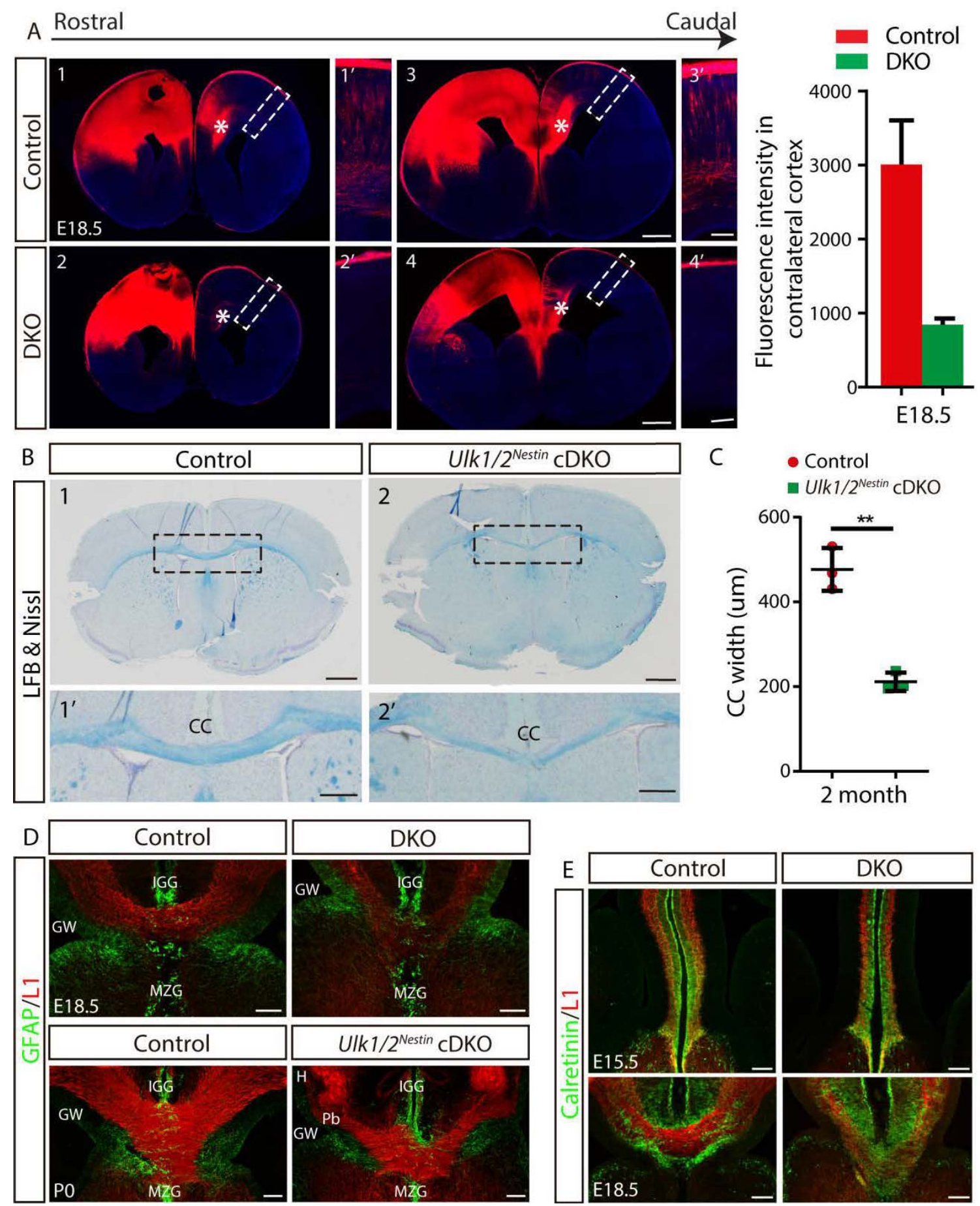

MZG

CC 
glial structures, including the glial wedge, indusium griseum glia, and midline zipper glia (Figure 4-6D). The guidepost neurons marked by calretinin appeared normal in the mutants at E15.5; thus, their abnormal distribution along the midline at E18.5 was more likely a consequence of improper positioning of the axons crossing the midline rather than the cause (Figure 4-6E).

\section{Ulk1/2-Deficiency Impairs Organization of the Somatosensory Cortex}

We next sought to characterize the axon guidance defects in Ulk1/2-deficient CTAs and TCAs in more detail. First, we embedded crystals of the fluorescent carbocyanine dye Dil into the presumptive somatosensory cortex of E18.5 embryos to label CTAs and trace their projection. Whereas the Dil crystals labeled the highly organized CTAs crossing the PSPB in control brains, the axons tended to traverse the striatum aberrantly in $U l k 1 / 2-D K O$ brains (Figure 4-7A1, A1', A3, A3'). Despite the disorganization and overfasciculation of the axons, the CTAs still found and targeted their final destination in the dorsal thalamus (dTh), as evidenced by the Dil signal in the dTh of $U l k 1 / 2$-DKO brains (Figure 4-7A2, A4). We also immunostained the brains of Ulk1/2 Nestin $\mathrm{cDKO}$ mice and controls at P21 for vesicular glutamate transporter 2 (VGluT2) and found no obvious differences in the intensity or distribution of this presynaptic marker in the dTh (Figure 4-7B). Cytochrome oxidase (CO) staining highlighted the intact array of barreloids in the thalamus (Figure 4-7B). Therefore, despite the abnormal fasciculation and path taken by the CTAs, we did not detect gross abnormalities in postnatal circuitry in the dTh.

We then embedded Dil crystals into the dTh of E18.5 to trace TCA projections. Similar to the CTAs, the TCAs were disorganized and overfasciculated as they crossed the PSPB (Figure 4-7C). TCAs project to the cortex through the intermediate zone during embryonic development and migrate radially to Layer IV at birth (Lopez-Bendito and Molnar, 2003). The extensive TCA arbors segregate into clusters surrounded by rings of Layer IV neurons' somas, whose dendrites are oriented to synapse with TCA afferents. Thalamic innervations are essential to remodel cortical neurons postnatally, and this organization gives rise to the barrels in the somatosensory cortex, which relay information from a single whisker (Lopez-Bendito and Molnar, 2003). The decreased levels of cortical staining in E18.5 Ulk1/2-DKO mice using an antibody against the TCA marker calretinin suggested that fewer TCAs reached cortex after crossing the PSPB than in controls (Figure 4-7D). The organization of the TCA projections postnatally in the brains of P7 mice was assessed by immunolabeling for the cortical barrel markers SERT and VGluT2 (Figure 4-7E, F). The somatosensory map in the Ulk1/2 ${ }^{\text {Nestin }}$ cDKO mice as revealed by $\mathrm{CO}$ stain was highly disorganized compared to that in the controls (Figure 4-7G). 
Figure 4-7. ULK1 and ULK2 are required for proper organization of the somatosensory cortex.

(A) Representative images of fluorescence signal from Dil crystals embedded in the somatosensory cortex reveals the highly disorganized of CTA projections crossing the PSPB in E18.5 Ulk1/2-DKO brains. These Ulk1/2-deficient axons, despite their disorganization and abnormal fasciculation, ultimately reach their targeted destination and innervate the dorsal thalamus (A2, A4). (B) Normal VGluT2 staining and cytochrome oxidase $(\mathrm{CO})$ staining in the dorsal thalamus in the Ulk1/2-DKO brains at P21 suggests proper cortical innervation of that structure. (C) Dil tracing shows the severely disorganized TCA projections in the Ulk1/2-DKO brains. (D) Representative image of Calretinin staining reveal a dramatic reduction in the number of TCA projections reaching the cortex in E18.5 Ulk1/2-DKO brains. (E-F) Representative images of sections immunostained using antibodies against the cortical barrel markers SERT (pseudocolored red), VGluT2 (pseudocolored green) (E), and the number of barrel units per section was quantified from control $(\mathrm{n}=3)$ and $U l k 1 / 2^{\text {Nestin }} \mathrm{cDKO}(\mathrm{n}=3)$ brains at P7 (F). (G) CO reveal disorganization of the somatosensory barrel cortex in P7 Ulk1/2 ${ }^{\text {Nestin }}$ cDKO brains. ${ }^{* *} P<0.01$. Abbreviations: dTh: dorsal thalamus; A: anterior; M: medial; VPL: ventral posterolateral nucleus; VPM: ventral posteromedial nucleus. Scale bars: $100 \mu \mathrm{m}$ in A1'-A3', B, C2'-C4', D1'-D2', E, and G; $500 \mu \mathrm{m}$ in A1-A4, C1C4, and D1-D2. 

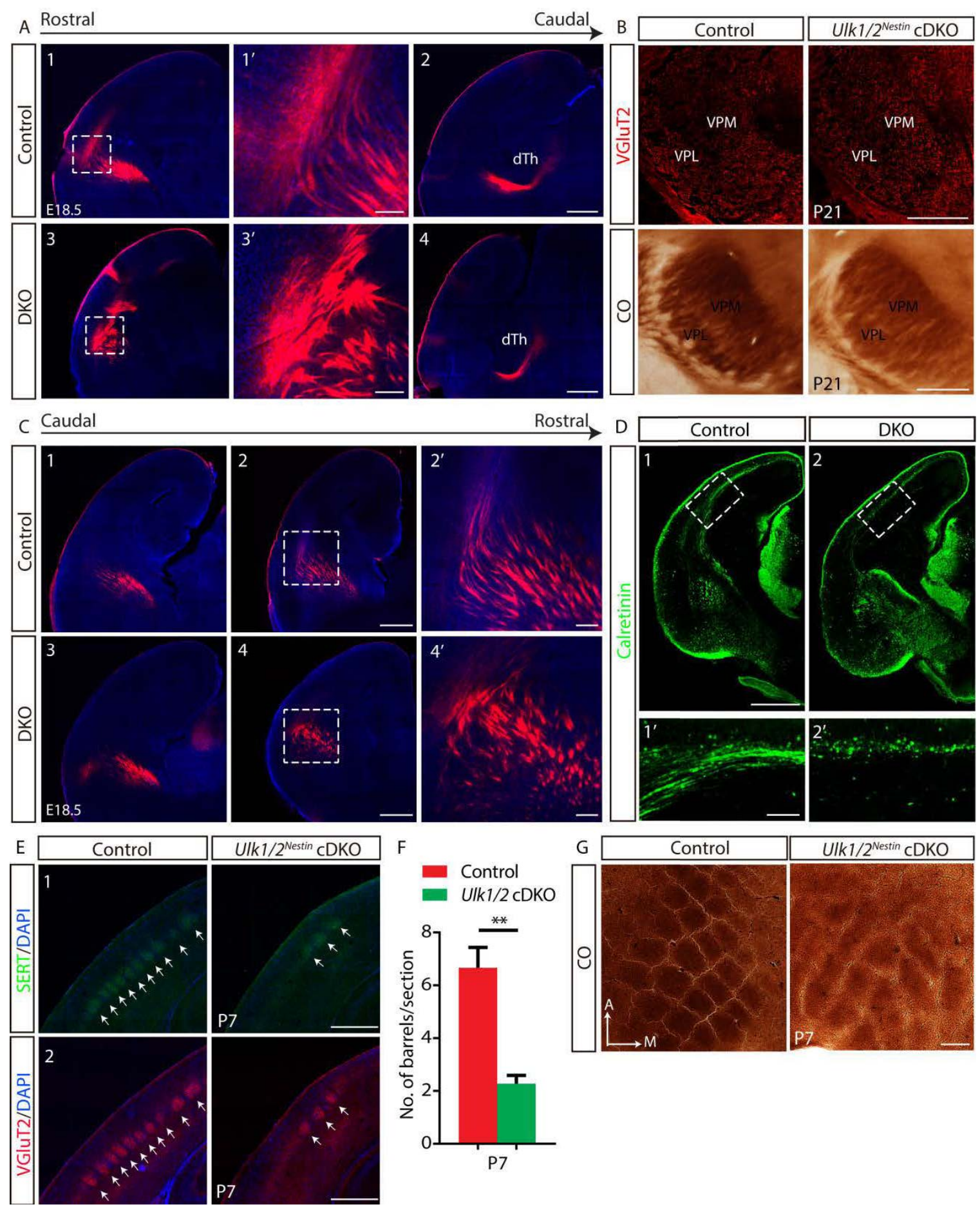


\section{TAG-1 Is Mislocalized in Corticothalamic Axons}

The defasciculation defects resulting from $U l k 1 / 2$-deficiency were similar to those observed in unc-51-or atg 1 -mutant invertebrates. In unc-51-mutant C. elegans, the axon guidance receptor UNC-5 is mislocalized to the cell soma and fails to mediate repulsion of axons away from the netrin-rich ventral midline.(Ogura and Goshima, 2006) In Drosophila, atgl regulates axonal localization of Fasciclin II, an IgG-family cell adhesion molecule (CAM), which is important for axonal guidance and fasciculation in the mushroom body. Therefore, we hypothesized that ULK1 and ULK2 may also regulate cellular localization of specific axon guidance receptors in projection neurons. Although most of the axon guidance receptors that we examined, including DCC, ROBO1, PSANCAM, and neural cell adhesion molecule (NCAM), were clearly present in the distal segments of projecting CTAs of both $U l k 1 / 2-\mathrm{DKO}$ and littermate controls (Figure 4-8A and data not shown), transient axonal glycoprotein-1 (TAG-1) was notably absent from the distal terminus of the CTAs in Ulk1/2-DKO embryos (Figure 4-8B). The expression of TAG-1 in the dorsal cortex appeared to be unaltered. We also dissected cortex (proximal CTAs) and striatum (distal CTAs) of E18.5 brains, and western blot analyses clearly confirmed the immunostaining results that TAG-1 level is dramatically reduced in the distal CTAs of the DKO animals (Figure 4-8C). These results indicate that ULK1 and ULK2 may regulate the trafficking of specific axon guidance molecules, such as TAG-1 in CTAs, in developing mouse brains similar to ULK/ATG1 homologues in invertebrates. 

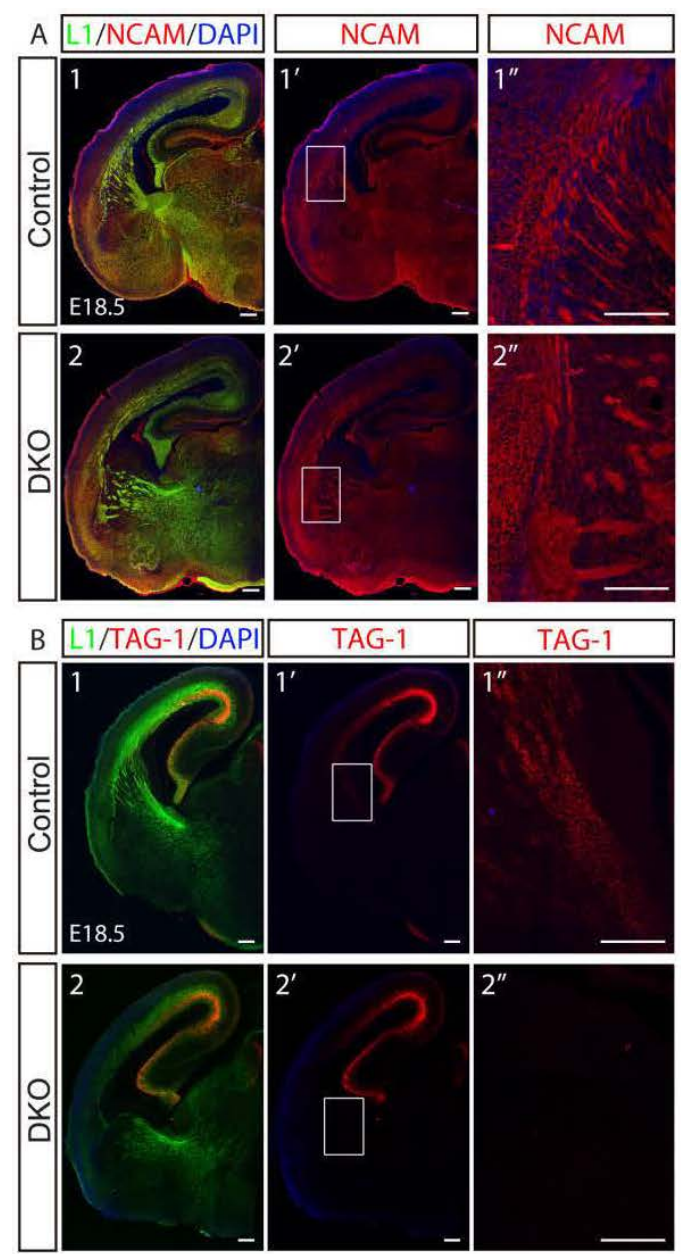

C

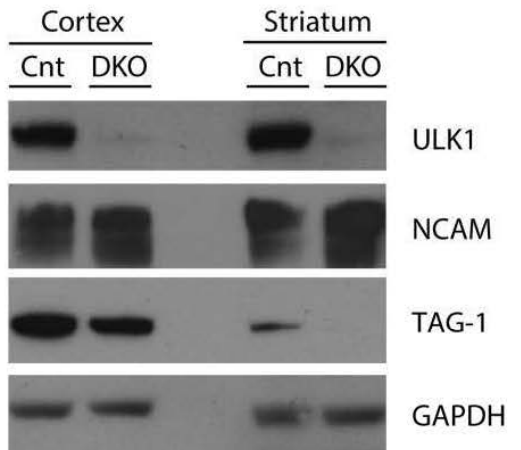

Figure 4-8. Abnormal axonal fasciculation in the $U l k 1 / 2$-deficient animals is associated with mislocalization of TAG-1 in the projection neurons.

(A) NCAM distribution was unaltered in the Ulk1/2-DKO brains compared to the controls. (B) The intensity of TAG-1 immunostaining was dramatically decreased in distal CTAs of the Ulk1/2-DKO brain at E18.5 compared to the controls. All of the sections were counterstained with L1. (C) Western blot analyses on the extracts prepared from cortex (proximal CTAs) and striatum (distal CTAs) confirmed normal expression of NCAM but decreased TAG-1 level in the striatum of the Ulk1/2-DKO brain. Scale bars: $200 \mu \mathrm{m}$. 


\section{CHAPTER 5. DISCUSSION*}

\section{The Role of ULK/ATG1 in ER-to-Golgi Trafficking Is Essential for Cellular Homeostasis}

Through genetic and biochemical approaches, we revealed an unexpected, evolutionarily conserved role for ULK/ATG1 in ER-to-Golgi trafficking under basal conditions, which is essential for maintaining cellular homeostasis. ULK1 and ULK2 play important roles in selective and nonselective autophagy induced in response to mitochondrial damage, infection, proteotoxic stress, or various forms of metabolic stress (Joo et al., 2011; Lim et al., 2015; Noda and Fujioka, 2015; Wong et al., 2013). Given the presumed importance of the ULKs in both forms of autophagy, the lack of ubiquitin ${ }^{+}$and $\mathrm{P} 2^{+}$inclusions or accumulation of abnormal mitochondria in the neurons of Ulk1/2-cdko animals was surprising. These results suggest that unlike ATG5, ATG7, and FIP200, all of which mediate the degradation of ubiquitinated proteins in neurons under basal physiologic conditions (Hara et al., 2006; Komatsu et al., 2006; Liang et al., 2010), ULK1 and ULK2 are not required for "basal" autophagy. Although we cannot exclude the possibility that another ULK/ATG1 homolog (e.g., ULK3) compensates for the lack of ULK1 and ULK2 in CNS neurons, our findings raise the possibility that an ATG13/FIP200 complex regulates the autophagy-mediated turnover of ubiquitinated proteins in an ULK/ATG1-independent manner. Recent studies in isolated cells have shown that ATG13 and FIP200 support starvation-induced autophagy in the absence of ULK1/2, albeit to a lesser extent than in their presence (Alers et al., 2011; Hieke et al., 2015). Such a pathway may support sufficient levels of autophagy in neurons under basal physiologic conditions to prevent the accumulation of ubiquitinated protein deposits.

Although ULK1 and ULK2 are not required for basal autophagy in neurons, our data indicate that a primary function of the ULKs in the absence of metabolic stress is regulating ER-to-Golgi trafficking. The CNS is spared in response to most metabolic stresses, perhaps making it one of a few environments in which the ULKs' role in ER-toGolgi trafficking can be readily separated from that in autophagy. ULK/ATG1-mediated COPII transport does not require the presence of other autophagy-related proteins (e.g., ATG13, ATG14, and ATG7), confirming that ULK/ATG1-regulated ER-to-Golgi trafficking is not an indirect consequence of ULKs affecting autophagy. Moreover, the absence of ATG13 does not disrupt the ULK1-SEC16A complex, as it does the canonical ULK/ATG1 complex composed of ULK1, ATG13, FIP200, and ATG101. These results indicate that regulating ER-to-Golgi trafficking under basal physiologic conditions is a noncanonical function (i.e., one that does not require ATG13) of the ULKs.

\footnotetext{
* Modified with permission. Joo, J.H., Wang, B., Frankel, E., Ge, L., Xu, L., Iyengar, R., Li-Harms, X., Wright, C., Shaw, T.I., Lindsten, T., et al. (2016). The Noncanonical Role of ULK/ATG1 in ER-to-Golgi Trafficking Is Essential for Cellular Homeostasis. Mol. Cell 62, 491-506.
} 
The uncoordinated phenotype of $C$. elegans unc-51 mutants is associated with defective axon guidance and is not recapitulated in autophagy-deficient mutants, such as epg-1 (Atg13) and epg-9 (Atg101) mutants (Liang et al., 2012; Tian et al., 2009). RNAimediated silencing of other autophagy-related genes, e.g., bec-1 (Atg6), M7.5 (Atg7), lgg1 (Atg8), or F41E6.5 (Atg18), also does not replicate the neuronal defects observed in unc-51 mutants (Ogura and Goshima, 2006). Our data suggest that the N-terminal domain of ULK/ATG1 and the central domain of SEC16A, which are the most highly evolutionarily conserved regions of both proteins, mediate the interaction between ULK/ATG1 and SEC16A. Indeed, the role of ULK/ATG1 in ER-to-Golgi trafficking appears to be conserved in C. elegans, as $u n c-51$ mutants show evidence of abnormal COPII assembly and trafficking of MOD-5 (SERT). Therefore, it is tempting to speculate that the defect in ER-to-Golgi trafficking in unc-51 mutants contributes to their uncoordinated phenotype.

Although the noncanonical function of ULKs in ER-to-Golgi trafficking does not require other autophagy-related proteins, recent studies have implicated the COPII pathway in autophagosome biogenesis (Ge et al., 2013; Ge et al., 2014; Graef et al., 2013; Ishihara et al., 2001; Suzuki et al., 2013). Given that ULK/ATG1 activity is regulated by energy/nutrient availability, we speculate that the ULK/ATG1-SEC16A interaction represents a specific point of convergence among the metabolism, autophagy, and COPII-transport pathways.

\section{ULK1/2 Are Essential for Axon Guidance in the Developing Mouse Forebrain}

Based on the observation that around $40 \%$ Ulk1/2 cdko mice die shortly after birth, and C. elegans UNC-51 and D. melanogaster Atg1 promote axonal development, we examined the potential embryonic neurodevelopmental defects in the Ulk1/2 cdko mice. We uncovered an evolutionarily conserved role of ULK $1 / 2$ in mammalian neurodevelopment. Using both germ-line knockout and conditional knockout mouse models, we demonstrated that loss of both Ulk1 and Ulk2 expression in the CNS led to pathfinding defects affecting callosal axons, AC, CTAs, TCAs, and mossy fibers via a non-canonical (i.e. autophagy-independent) pathway. The autophagy-inducing activity of ULK/ATG1 depends on its stable interaction with ATG13 and FIP200 (Ganley et al., 2009; Hosokawa et al., 2009; Jung et al., 2009), and can be regulated by AMBRAmediated ubiquitinylation of ULK1 (Nazio et al., 2013). AMBRA1 is required for neuronal differentiation and survival during early embryogenesis, and FIP200 is required for maintenance of cortical neurons and neuronal stem cells postnatally (Wang et al., 2013). Although a kinase complex composed of ULK1, ATG13 and FIP200 was detected in both $\mathrm{P} 0$ and adult cortex, we failed to observe excessive neuronal cell death in $U l k 1 / 2$ deficient embryos, accumulation of $\mathrm{p} 62^{+}$and ubiquitin ${ }^{+}$deposits, or loss of cortical neurons or neuronal stem cells in Ulk1/2-deficient adults. Moreover, the neurodevelopmental abnormalities found in $U l k 1 / 2$-deficient mice were not recapitulated in mice lacking expression of Fip200 (or Atg7) in the CNS. Together, these findings are consistent with the notion that autophagy proceeds in the absence of ULK $1 / 2$ in neurons under basal physiologic condition (Joo et al., 2016) and that ULK1/2 regulate axon 
guidance via autophagy-independent pathway(s). Similar results were observed in $C$. elegans, where the axon guidance and defasciculation defects observed in unc-51 mutants were not recapitulated in epg-1 (Atg13) and epg-9 (Atg101) mutants (Liang et al., 2012; Tian et al., 2009).

Our studies demonstrated that ULK1 and ULK2, similar to their counterparts in C. elegans and D. melanogaster, are essential for proper axon guidance and defasciculation in mammals in vivo. C. elegans unc-51 mutant neurons display multiple axonal defects, including premature termination, abnormal trajectories, and extra axonal branches (Ogura et al., 1994). UNC-51 binds to UNC-14, a RUN-domain protein that acts as an effector of Rap and Rab GTPases and regulates membrane trafficking (Lai and Garriga, 2004; Ogura et al., 1997). Disruption of either unc-14 or unc-51 expression leads to aberrant localization of UNC-5 to the cell soma rather than axons, and a failure to repulse axons away from the netrin-rich ventral midline (Ogura and Goshima, 2006). UNC-51 also interacts with VAB-8, which controls the cell-surface expression of SAX3/Robo in touch neuron growth cones (Lai and Garriga, 2004; Watari-Goshima et al., 2007). Thus, the defect in axon guidance in C. elegans unc-51 mutants is at least in part due to disruption of the UNC-51/ RUN-14/ VAB-8 complex, which regulates the trafficking of various axon guidance molecules to axons (Ogura and Goshima, 2006). Similarly, the axon guidance molecule Fasciclin II, which regulates fasciculation of mushroom body axons in D. melanogaster, is mislocalized in atgl mutants (Mochizuki et al., 2011). In mammals, ULK1 interacts with the synaptic GTPase-activation protein SynGAP, and Syntenin, both of which regulate Rab5-mediated neuronal endocytotic pathway (Tomoda et al., 2004). ULK1 and ULK2 have been implicated in TrkA-receptor trafficking and the endocytosis of nerve growth factor (Zhou et al., 2007). ULK/ATG1 also has a conserved role in regulating assembly of specific ER exit sites (i.e. SEC24C+) and trafficking of associated cargo (Joo et al., 2016). Together, ULK/ATG1-mediated trafficking of axon guidance molecules contributes to the axonal development from $C$. elegans to mammals.

TAG-1 is a neural cell adhesion molecule that is expressed during brain development and has been implicated axon outgrowth, neurite extension and fasciculation, and neuronal migration during development (Karagogeos, 2003). Nevertheless, the precise consequence of the mislocalized TAG-1 in Ulk1/2-deficient animals remains unclear. Among the cargoes whose ER-to-Golgi trafficking is regulated by ULK1/2 is the serotonin transporter, SERT (Joo et al., 2016). SERT is transiently expressed in TCAs, where it helps to regulate serotonin levels (Chen et al., 2015). The appropriate levels of serotonin at synaptic junctions is critical for the normal organization and development of the thalamocortical afferent circuits, and impaired uptake of the serotonin due to genetic or pharmacologic disruption of SERT function in TCAs impairs barrel cortex formation (Chen et al., 2015; Miceli et al., 2013). The disorganization of the somatosensory cortex in $U l k 1 / 2$ cdko is similar to (albeit more severe than) that resulting from genetic or pharmacologic disruption of SERT activity in developing mouse brains (van Kleef et al., 2012). Therefore, we speculate that ULK1 and ULK2, similar to their invertebrate homologues, regulate one or more steps in the trafficking of multiple secretory cargoes, TAG-1 and SERT among them, from the ER to the PM, and that 
defects in trafficking contribute to the defects in axon guidance in the brains of developing Ulk1/2-deficient animals.

\section{Emerging Noncanonical Functions of ULK/ATG1}

Our studies have uncovered two important noncanonical functions of ULK1/2 in the mouse brain under basal physiological condition. Complementary to our findings, evidence has also accumulated over the years that ULK/ATG1 regulates cellular trafficking under normal physiological conditions, which appears to be autophagyindependent. Moreover, recent data have established that ULK1 and ULK2 are essential for innate immunity, cell death, and glycolysis following starvation (Figure 5-1). As studies on ULK/ATG1 continue, more noncanonical functions of ULK/ATG1 are likely to be revealed in the future.

\section{ULK1/2 Regulate Metabolism}

Although ULK1 and ULK2 are important integrators relaying metabolic stress signals to downstream autophagy machinery, whether ULK1 and ULK2 modulate other metabolic pathways, such as glucose metabolism, remains unclear. A recent study demonstrated that during AA and serum starvation, ULK1/2 phosphorylate key glycolytic enzymes, including hexokinase (HK), phosphofructokinase 1 (PFK1), enolase 1 (ENO1), and the gluconeogenic enzyme fructose-1,6-bisphosphatase (FBP1) to secure more carbon flux into the pentose phosphate pathway and maintain cellular energy supply ( $\mathrm{Li}$ et al., 2016). More importantly, knockdown of other key autophagy genes, including Atg5, Atg7, and Becn 1 had no effect on glucose consumption under the same conditions (Li et al., 2016), indicating that driving glucose metabolism, in addition to initiating autophagy to maintain sufficient intracellular energy level, is a unique feature of the ULK kinases compared to other autophagy proteins.

Intriguingly, another study revealed that $U l k 1$ knockdown not only compromised basal and rapamycin-induced autophagy but also reduced fatty acid oxidation and enhanced fatty acid uptake at the steady state in the differentiated adipocytes, and knockdown of Ulk2 produced the opposite effect, compared to that of Ulk1 (Ro et al., 2013). Meanwhile, expression levels of insulin receptor, insulin receptor substrate 1, and glucose transporter were all increased in Ulkl-depleted adipocytes (Ro et al., 2013). However, the relevant substrate of ULK1 and ULK2 remains to be discovered, the physiological relevance of Ro et al.'s study waits to be confirmed, and the mechanisms underlying the mutually opposite functions of ULK1 and ULK2 need to be revealed. In any case, these preliminary studies opened up a new area where ULK1 and ULK2 emerge as key regulators in various metabolic pathways, such as glucose breakdown and fatty acid oxidation, among others, under different nutrient conditions. 


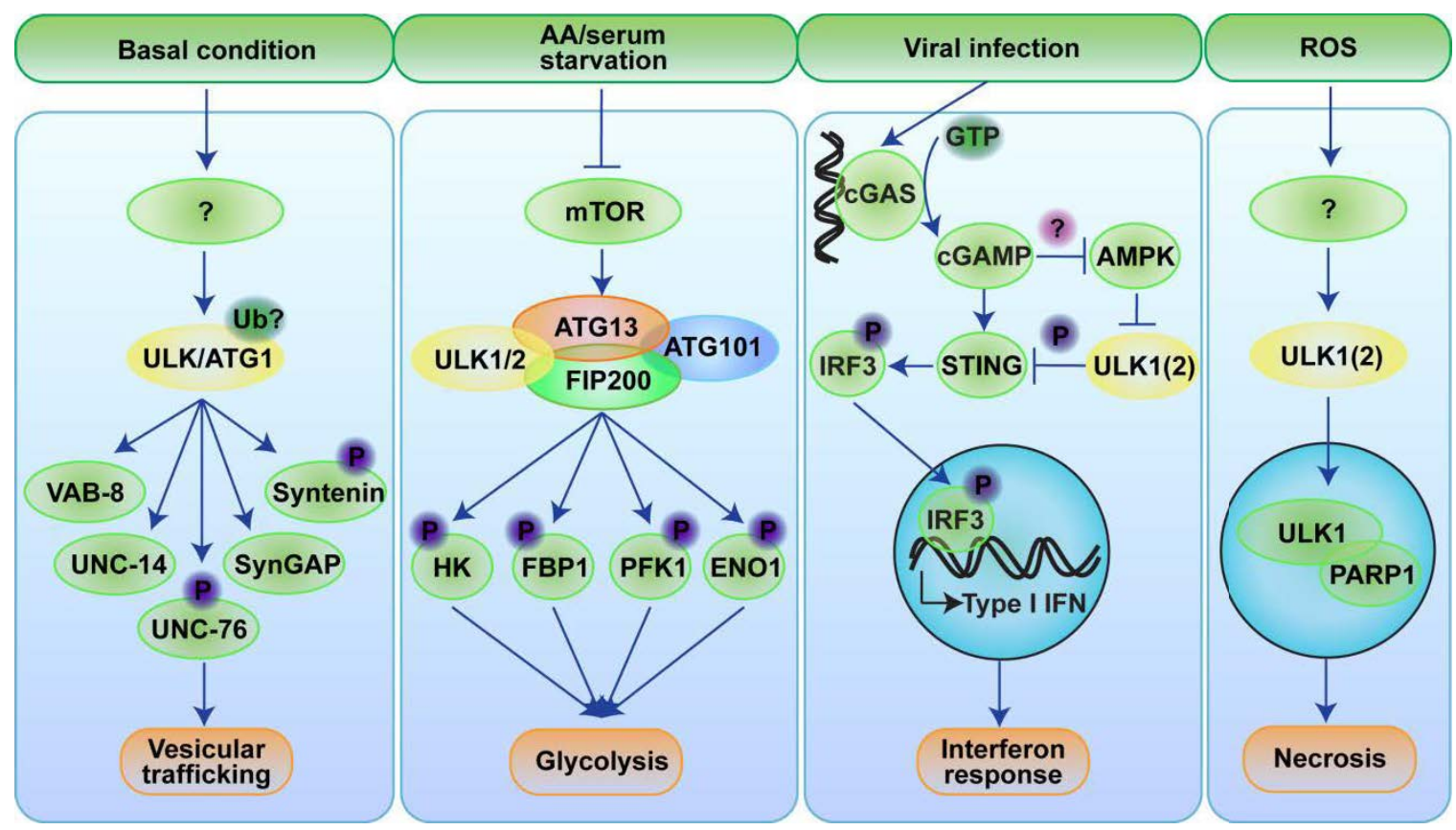

Figure 5-1. The versatile functions of ULK/ATG1 beyond autophagy regulation. Depending on the cellular physiological status, ULK/ATG1 is capable of targeting different molecules to dictate cell fate. Under normal growth conditions, such as embryonic development, ULK/ATG1 binds to myriad proteins, such as $C$. elegans UNC14 and VAB-8, D. melanogaster UNC-76, and mammalian SynGAP and Syntenin, to mediate vesicle trafficking in neurite outgrowth. Ubiquitilation of UNC-51, triggered by vesicle cargo such as NGF and TrkA, can potentially regulate the function of ULK/ATG1. Upon AA/serum starvation, ULK1/2 phosphorylate key enzymes of glycolysis, including HK, FBP1, PFK1, and ENO1, to ensure sufficient energy supply in addition to autophagy regulation, which is essential for cell survival. Once detected, viral DNA binds to and activates cGAS to synthesize cGAMP. As a second messenger, cGAMP, on the one hand, activates STING to promote IRF3-dependent transcription of IFNs. On the other hand, cGAMP inhibits AMPK by an unknown mechanism and subsequently activates ULK1 to phosphorylate STING. Phosphorylation of STING by ULK1 suppresses its ability to initiate transcription of IFNs, thus providing a negative feedback mechanism to prevent excessive immune response. Sustained stress imposed by ROS leads to translocation of ULK1 from cytosol to nucleus, where ULK1 binds and modulates PARP1 function to promote necrotic cell death. See text for details. 


\section{Vesicular Trafficking}

Another important aspect of ULK/ATG1's function lies in vesicular trafficking. C. elegans UNC-51 directly binds to UNC-14, a RUN-domain protein that acts as an effector of Rap and Rab GTPases and modulates membrane trafficking (Ogura and Goshima, 2006; Ogura et al., 1994). In unc-14 and unc-51 mutants, UNC-5 is aberrantly localized to the cell soma rather than axons (Ogura and Goshima, 2006). The interaction between UNC-51 and the novel protein VAB-8 also implicates UNC-51 in regulating guidance-receptor trafficking, considering that VAB-8 controls the cell-surface expression of SAX3/Robo in touch neuron growth cones (Lai and Garriga, 2004; WatariGoshima et al., 2007). Although there seems to be UNC-51-dependent phosphorylation of both UNC-14 and VAB-8, the mechanism underlying the regulation of UNC-14 and VAB-8 is not yet clear. The Drosophila atg 1 mutants exhibit abnormalities during embryonic formation of axonal tracts similar to unc-51 mutant (Mochizuki et al., 2011; Toda et al., 2008). ATG1 physically binds and phosphorylates the kinesin adaptor UNC76 at Ser143. The phosphorylated UNC-76 has an elevated affinity for Synaptotagmin-1, a major transmembrane protein of synaptic vesicles (Toda et al., 2008). More importantly, the defective axonal transport of synaptic vesicles in unc-76 mutants is rescued by a phospho-mimetic UNC-76 (S143D), highlighting the essential role of ATG1-mediated phosphorylation of UNC-76 in axonal transport (Toda et al., 2008). In mammals, early studies identified the synaptic GTPase-activation protein (SynGAP) and Syntenin, an endocytic vesicular membrane protein with PDZ domains, as novel binding partners of ULK1; both proteins regulate the Rab5-mediated neuronal endocytotic pathway (Tomoda et al., 2004). Moreover, ULK1 and ULK2 regulate TrkA-receptor trafficking and the endocytosis of nerve growth factor in mouse dorsal root ganglion neurons in culture (Zhou et al., 2007). Mechanistically, NGF can induce the interaction of ULK1 with TrkA receptor complexes through promoting K63-polyubiquitination of ULK1 and binding ULK1 to the scaffolding protein p62 (Zhou et al., 2007). It is unclear whether ULK1 phosphorylates any of the homologous binding partners in mammals, and the physiological relevance of these interactions needs further investigation.

\section{Interferon Response}

Pathogenic nucleic acids, such as DNA, RNA, and nucleotides, are detected by host sensing mechanisms (Wu and Chen, 2014). These cell surface sensors regulate activation of several downstream effectors that lead to the transcription of type I IFNs and pro-inflammatory cytokines that regulate innate and adaptive immune responses $(\mathrm{Wu}$ and Chen, 2014). IFNs are then secreted and bind cell surface receptors where they initiate transcription of interferon-stimulated genes (ISGs) to mediate appropriate IFN response and anti-viral effect (Ivashkiv and Donlin, 2014). Recent studies have implicated ULK1 and ULK2 in IFN production as well as IFN signaling. Following exposure of cytosolic DNA, the DNA-binding protein cyclic guanosine monophosphateadenosine monophosphate (cGAMP) synthase (cGAS) triggers synthesis of cGAMP to activate stimulator of interferon genes (STING), which then phosphorylates interferon regulatory factor 3 (IRF3), the transcription factor directly involved in transcription of 
IFNs (Wu et al., 2013). Meanwhile, cGAMP is required for dephosphorylation of AMPK at Thr172 and subsequent dephosphorylation of Ser555 of murine ULK1. This also leads to activation of ULK1, which directly phosphorylates STING at Ser366 to block its kinase activity towards IRF3 and subsequently inhibits transcription of related immune genes (Konno et al., 2013). Thus, ULK1 fine tunes the immune response to pathogenic nucleic acids through a negative-feedback mechanism to prevent STING-dependent inflammatory disorders. Interestingly, another study reported that upon IFN stimulation, AKT-dependent phosphorylation of ULK1 at Ser757 is required for phosphorylation of p38 MAPK and eventually proper transcription of ISGs in multiple cell lines (Saleiro et al., 2015). More importantly, abolishing ATG5 had no impact on the transcription of ISGs, suggesting that ULK1 and ULK2 mediate IFN response, probably via a noncanonical, autophagy-independent mechanism (Saleiro et al., 2015). Notably, patients with myeloproliferative neoplasms (MPNs) were found to have elevated expression levels of ULK1 compared to controls, and suppression of ULK1 expression blocked the anti-neoplastic functions of type I IFNs against malignant erythroid precursors derived from the patients (Saleiro et al., 2015). These results suggest that increased ULK1 expression could correlate with favorable responses to type I IFN therapy in MPNs and other types of IFN-responsive cancers, but this remains to be explored further.

\section{Necrotic Cell Death}

Generally perceived as a pro-survival mechanism, autophagy can also be destructive and lead to cell death under certain conditions (Maiuri et al., 2007). How individual autophagy machinery, including ULK1 and ULK2, contributes to cell death remains largely unknown. A previous study suggested that treating osteosarcoma cells with sub-lethal dose of camptothecin leads to p53-mediated upregulation of ULK1 at transcription level, which induced sustained autophagy and subsequently cell death (Gao et al., 2011). Our recent study for the first time has demonstrated that ULK1 translocates to the nucleus and sensitizes cells to necrotic cell death in response to hydrogen peroxide treatment (Joshi et al., 2016). Mechanistically, nuclear ULK1 interacts with poly (ADPribose) polymerase 1 (PARP1) and enhances PARP1 activity, which accelerates ATP depletion and cell death in an autophagy-independent manner (Joshi et al., 2016). Whether PARP is a novel substrate of ULK1 or there is an unknown substrate of ULK1 bridging ULK1 and PARP, and the signaling events leading to the translocation of ULK1 from cytosol to nucleus need further investigation. Interestingly, ULK2 has also been shown to localize to the nucleus with the aid of an importer receptor-Kap $\beta 2$, even under basal conditions in HEK293 cells. The functional significance of the nuclear ULK2 has not yet been elucidated (Shin et al., 2015).

\section{ULKs and Neurological Diseases}

For neurons, eliminating dysfunctional organelles and cellular waste over a lifetime without harnessing cell division to buffer the waste burdens is particularly challenging (Nixon, 2013). Hence, neurons rely heavily on autophagy for survival, and 
dysregulated autophagy is often linked to neurodegenerative diseases (Nixon, 2013). As expected, conditionally knocking out Atg5, Atg7, or Fip200 in the CNS causes widespread neuronal loss, associated with accumulation of p62- and ubiquitin-positive inclusion bodies, a pathological feature shared by numerous human neurological diseases (Hara et al., 2006; Komatsu et al., 2006; Liang et al., 2010; Nixon, 2013). Our study suggests that, unlike other canonical autophagy proteins, ULK1 and ULK2 are not required for "basal" autophagy in the neurons (Joo et al., 2016). However, the existence of stable ULK1-ATG13-FIP200 in the cortex at various ages suggests that ULK1 and/or ULK2 might induce autophagy in response to certain stresses, such as aberrant accumulation of protein aggregates. This hypothesis is supported by a recent study, which demonstrated that in response to proteasome inhibition or expression of polyQ-Htt, ULK1 phosphorylates the autophagy receptor protein p62 thus inducing autophagymediated degradation of excessive protein aggregates (Lim et al., 2015). Evidence that dysfunction of ULKs contributes to human neurological diseases is sparse. A recent study revealed that both ULK1 and ULK2, together with other autophagy proteins, accumulate in the mature Lewy bodies in the brains of patients with Parkinson's disease or dementia with Lewy bodies (Miki et al., 2016). This likely represents a cellular protective mechanism in which ULK1 and ULK2 cooperate to degrade the excessive aggregates of disease proteins through autophagy, rendering ULK1 and ULK2 attractive targets for treating certain neurodegenerative diseases. Alternatively, ULK1- and ULK2-mediated cellular trafficking may affect the developing brain. Brain wiring abnormalities are believed to contribute significantly to a spectrum of psychological diseases, including the ones with a neurodevelopmental origin (autism, for example). No mutations in Ulk1 or Ulk2 have so far been reported in human patients suffering from neurological disorders. However, similar to our observations in ULK1/2 deficient mice, where the survival rate is lowered, Ulk1 or Ulk2 mutations may cause early mortality in humans too.

\section{Future Directions}

Recent work from several labs, including our own, has provided significant insights on the cellular functions and the regulation of ULKs. The physiological significance of ULKs in vivo, however, still remains largely unclear, thus calling for more detailed analyses of Ulk-deficient mouse models. As it is possible that the in vivo function of ULKs might be tissue-dependent, it is essential that the phenotypic analyses on the Ulk-deficient animals be performed under basal conditions with minimal bias. It will also be crucial to ascertain whether the phenotype is autophagy-dependent or independent by comparing Ulk-deficient animals with mice depleted of other autophagy genes such as Atg7, and Fip200. Despite the well-established significance of ULKs in metabolic stress-induced autophagy in several cell lines, investigations on in vivo models may yield different results, leading to different conclusions. Thus, it is prudent to use multiple means and modes of stress (such as starvation and ischemia-reperfusion) to induce autophagy in Ulk-deficient mice and their effects should be studied.

Given the plethora of cellular processes that ULKs may be involved in, it is reasonable to predict that there are more substrates of these kinases than those 
summarized in Chapter 1. Thus, using different approaches to identify novel substrates of ULKs will be of continuous interest. Not only do the phosphorylation sites of these potential substrates need to be located with traditional biochemical assays, but also the possible functional significance of these interactions and phosphorylations needs to be explored. In addition, identifying novel upstream regulators of ULKs will be critical to understanding how ULKs integrate the signals and dictate the cellular response under different conditions. Another puzzle to solve is how the function of ULKs is coordinated in different environments. It is possible that under stress conditions, ULKs are hijacked into the autophagy pathway by having their normal function compromised. Alternatively, the noncanonical function of ULK/ATG1 may also contribute directly to autophagy. This notion is validated by recent reports that the ER membrane is a source for autophagosome formation, and that the COPII pathway is involved in autophagosome biogenesis (Ge et al., 2013; Ge et al., 2014; Graef et al., 2013; Ishihara et al., 2001; Suzuki et al., 2013). Thus, it is tempting to speculate that ULK/ATG1-regulated ER-to-Golgi trafficking may act as a membrane source during autophagy induction. Nevertheless, identifying the upstream molecules that dictate the specific function of ULKs under different conditions remains an important but also challenging task.

Finally, disruption of ULK function may contribute to the pathogenesis of multiple human diseases, including cancer and neurodegeneration, which doesn't necessarily involve the autophagy-dependent mechanism. In light of our novel description of the noncanonical function of ULKs, it becomes necessary to revisit the previously established idea that ULKs function solely in an autophagy-dependent fashion in cancer cells. As the noncanonical function of ULKs could potentially also contribute to tumor cell growth or demise, depending on the specific context, it is therefore critical to separate the different function of ULKs in any specific situation. Now that the knockout mouse models of ULK1 and ULK2 are available, crossing the Ulk1 and Ulk2 KO mice with mouse models of cancer and neurological diseases and access the role of ULK1 and ULK in the disease progression will be an attractive direction to explore. This will not only facilitate a clearer understanding of the basic mechanisms of pathogenesis, but also will help develop new therapeutic strategies to combat many devastating diseases. 


\section{LIST OF REFERENCES}

Alers, S., Loffler, A.S., Paasch, F., Dieterle, A.M., Keppeler, H., Lauber, K., Campbell, D.G., Fehrenbacher, B., Schaller, M., Wesselborg, S., et al. (2011). Atg13 and FIP200 act independently of Ulk1 and Ulk2 in autophagy induction. Autophagy 7, 1423-1433.

Alers, S., Loffler, A.S., Wesselborg, S., and Stork, B. (2012). Role of AMPK-mTORUlk1/2 in the regulation of autophagy: cross talk, shortcuts, and feedbacks. Mol Cell Biol 32, 2-11.

Audhya, A., Hyndman, F., McLeod, I.X., Maddox, A.S., Yates, J.R., 3rd, Desai, A., and Oegema, K. (2005). A complex containing the Sm protein CAR-1 and the RNA helicase CGH-1 is required for embryonic cytokinesis in Caenorhabditis elegans. J Cell Biol 171, 267-279.

Bach, M., Larance, M., James, D.E., and Ramm, G. (2011). The serine/threonine kinase ULK1 is a target of multiple phosphorylation events. Biochem J 440, 283-291.

Bhattacharyya, D., and Glick, B.S. (2007). Two mammalian Sec16 homologues have nonredundant functions in endoplasmic reticulum (ER) export and transitional ER organization. Mol Biol Cell 18, 839-849.

Bonnon, C., Wendeler, M.W., Paccaud, J.P., and Hauri, H.P. (2010). Selective export of human GPI-anchored proteins from the endoplasmic reticulum. J Cell Sci 123, 1705-1715.

Brenner, B., Harney, J.T., Ahmed, B.A., Jeffus, B.C., Unal, R., Mehta, J.L., and Kilic, F. (2007). Plasma serotonin levels and the platelet serotonin transporter. J Neurochem 102, 206-215.

Budovskaya, Y.V., Stephan, J.S., Deminoff, S.J., and Herman, P.K. (2005). An evolutionary proteomics approach identifies substrates of the cAMP-dependent protein kinase. Proc Natl Acad Sci U S A 102, 13933-13938.

Chan, E.Y., Kir, S., and Tooze, S.A. (2007). siRNA screening of the kinome identifies ULK1 as a multidomain modulator of autophagy. J Biol Chem 282, 25464-25474.

Chen, X., Ye, R., Gargus, J.J., Blakely, R.D., Dobrenis, K., and Sze, J.Y. (2015). Disruption of Transient Serotonin Accumulation by Non-Serotonin-Producing Neurons Impairs Cortical Map Development. Cell Rep.

Cheong, H., Lindsten, T., Wu, J., Lu, C., and Thompson, C.B. (2011). Ammonia-induced autophagy is independent of ULK1/ULK2 kinases. Proc Natl Acad Sci U S A 108, 11121-11126.

Cheong, H., Wu, J., Gonzales, L.K., Guttentag, S.H., Thompson, C.B., and Lindsten, T. (2014). Analysis of a lung defect in autophagy-deficient mouse strains. Autophagy 10, 45-56.

Di Nardo, A., Kramvis, I., Cho, N., Sadowski, A., Meikle, L., Kwiatkowski, D.J., and Sahin, M. (2009). Tuberous sclerosis complex activity is required to control neuronal stress responses in an mTOR-dependent manner. J Neurosci 29, 59265937.

Egan, D.F., Chun, M.G., Vamos, M., Zou, H., Rong, J., Miller, C.J., Lou, H.J., Raveendra-Panickar, D., Yang, C.C., Sheffler, D.J., et al. (2015). Small Molecule 
Inhibition of the Autophagy Kinase ULK1 and Identification of ULK1 Substrates. Mol Cell 59, 285-297.

Egan, D.F., Shackelford, D.B., Mihaylova, M.M., Gelino, S., Kohnz, R.A., Mair, W.,

Vasquez, D.S., Joshi, A., Gwinn, D.M., Taylor, R., et al. (2011). Phosphorylation of ULK1 (hATG1) by AMP-activated protein kinase connects energy sensing to mitophagy. Science 331, 456-461.

Fang, J., Liu, M., Zhang, X., Sakamoto, T., Taatjes, D.J., Jena, B.P., Sun, F., Woods, J., Bryson, T., Kowluru, A., et al. (2015). COPII-Dependent ER Export: A Critical Component of Insulin Biogenesis and beta-Cell ER Homeostasis. Mol Endocrinol 29, 1156-1169.

Feng, Y., He, D., Yao, Z., and Klionsky, D.J. (2014). The machinery of macroautophagy. Cell Res 24, 24-41.

Fimia, G.M., Stoykova, A., Romagnoli, A., Giunta, L., Di Bartolomeo, S., Nardacci, R., Corazzari, M., Fuoco, C., Ucar, A., Schwartz, P., et al. (2007). Ambra1 regulates autophagy and development of the nervous system. Nature 447, 1121-1125.

Ganley, I.G., Lam du, H., Wang, J., Ding, X., Chen, S., and Jiang, X. (2009). ULK1.ATG13.FIP200 complex mediates mTOR signaling and is essential for autophagy. J Biol Chem 284, 12297-12305.

Gao, W., Shen, Z., Shang, L., and Wang, X. (2011). Upregulation of human autophagyinitiation kinase ULK1 by tumor suppressor p53 contributes to DNA-damageinduced cell death. Cell Death Differ 18, 1598-1607.

Ge, L., Melville, D., Zhang, M., and Schekman, R. (2013). The ER-Golgi intermediate compartment is a key membrane source for the LC3 lipidation step of autophagosome biogenesis. Elife 2, e00947.

Ge, L., Zhang, M., and Schekman, R. (2014). Phosphatidylinositol 3-kinase and COPII generate LC3 lipidation vesicles from the ER-Golgi intermediate compartment. Elife 3, e04135.

Graef, M., Friedman, J.R., Graham, C., Babu, M., and Nunnari, J. (2013). ER exit sites are physical and functional core autophagosome biogenesis components. Mol Biol Cell 24, 2918-2931.

Hara, T., Nakamura, K., Matsui, M., Yamamoto, A., Nakahara, Y., Suzuki-Migishima, R., Yokoyama, M., Mishima, K., Saito, I., Okano, H., et al. (2006). Suppression of basal autophagy in neural cells causes neurodegenerative disease in mice. Nature 441, 885-889.

Harding, T.M., Morano, K.A., Scott, S.V., and Klionsky, D.J. (1995). Isolation and characterization of yeast mutants in the cytoplasm to vacuole protein targeting pathway. J Cell Biol 131, 591-602.

Hieke, N., Loffler, A.S., Kaizuka, T., Berleth, N., Bohler, P., Driessen, S., Stuhldreier, F., Friesen, O., Assani, K., Schmitz, K., et al. (2015). Expression of a ULK1/2 binding-deficient ATG13 variant can partially restore autophagic activity in ATG13-deficient cells. Autophagy 11, 1471-1483.

Hosokawa, N., Hara, T., Kaizuka, T., Kishi, C., Takamura, A., Miura, Y., Iemura, S., Natsume, T., Takehana, K., Yamada, N., et al. (2009). Nutrient-dependent mTORC1 association with the ULK1-Atg13-FIP200 complex required for autophagy. Mol Biol Cell 20, 1981-1991. 
Ichimura, Y., and Komatsu, M. (2010). Selective degradation of p62 by autophagy. Semin Immunopathol 32, 431-436.

Ishihara, N., Hamasaki, M., Yokota, S., Suzuki, K., Kamada, Y., Kihara, A., Yoshimori, T., Noda, T., and Ohsumi, Y. (2001). Autophagosome requires specific early Sec proteins for its formation and NSF/SNARE for vacuolar fusion. Mol Biol Cell 12, 3690-3702.

Ivashkiv, L.B., and Donlin, L.T. (2014). Regulation of type I interferon responses. Nat Rev Immunol 14, 36-49.

Jafari, G., Xie, Y., Kullyev, A., Liang, B., and Sze, J.Y. (2011). Regulation of extrasynaptic 5-HT by serotonin reuptake transporter function in 5-HT-absorbing neurons underscores adaptation behavior in Caenorhabditis elegans. J Neurosci 31, 8948-8957.

Joo, J.H., Dorsey, F.C., Joshi, A., Hennessy-Walters, K.M., Rose, K.L., McCastlain, K., Zhang, J., Iyengar, R., Jung, C.H., Suen, D.F., et al. (2011). Hsp90-Cdc37 chaperone complex regulates Ulk1- and Atg13-mediated mitophagy. Mol Cell 43, $572-585$.

Joo, J.H., Wang, B., Frankel, E., Ge, L., Xu, L., Iyengar, R., Li-Harms, X., Wright, C., Shaw, T.I., Lindsten, T., et al. (2016). The Noncanonical Role of ULK/ATG1 in ER-to-Golgi Trafficking Is Essential for Cellular Homeostasis. Mol Cell 62, 491506.

Joshi, A., Iyengar, R., Joo, J.H., Li-Harms, X.J., Wright, C., Marino, R., Winborn, B.J., Phillips, A., Temirov, J., Sciarretta, S., et al. (2016). Nuclear ULK1 promotes cell death in response to oxidative stress through PARP1. Cell Death Differ 23, 216230.

Jung, C.H., Jun, C.B., Ro, S.H., Kim, Y.M., Otto, N.M., Cao, J., Kundu, M., and Kim, D.H. (2009). ULK-Atg13-FIP200 complexes mediate mTOR signaling to the autophagy machinery. Mol Biol Cell 20, 1992-2003.

Kaizuka, T., and Mizushima, N. (2015). Atg13 Is Essential for Autophagy and Cardiac Development in Mice. Mol Cell Biol 36, 585-595.

Kalabis, J., Rosenberg, I., and Podolsky, D.K. (2006). Vangll protein acts as a downstream effector of intestinal trefoil factor (ITF)/TFF3 signaling and regulates wound healing of intestinal epithelium. J Biol Chem 281, 6434-6441.

Kamada, Y., Funakoshi, T., Shintani, T., Nagano, K., Ohsumi, M., and Ohsumi, Y. (2000). Tor-mediated induction of autophagy via an Apg1 protein kinase complex. J Cell Biol 150, 1507-1513.

Kamada, Y., Yoshino, K., Kondo, C., Kawamata, T., Oshiro, N., Yonezawa, K., and Ohsumi, Y. (2010). Tor directly controls the Atg1 kinase complex to regulate autophagy. Mol Cell Biol 30, 1049-1058.

Karagogeos, D. (2003). Neural GPI-anchored cell adhesion molecules. Front Biosci 8, s1304-1320.

Kim, J., Kleizen, B., Choy, R., Thinakaran, G., Sisodia, S.S., and Schekman, R.W. (2007). Biogenesis of gamma-secretase early in the secretory pathway. J Cell Biol 179, 951-963.

Kim, J., Kundu, M., Viollet, B., and Guan, K.L. (2011). AMPK and mTOR regulate autophagy through direct phosphorylation of Ulk1. Nat Cell Biol 13, 132-141. 
Komatsu, M., Waguri, S., Chiba, T., Murata, S., Iwata, J., Tanida, I., Ueno, T., Koike, M., Uchiyama, Y., Kominami, E., et al. (2006). Loss of autophagy in the central nervous system causes neurodegeneration in mice. Nature 441, 880-884.

Konno, H., Konno, K., and Barber, G.N. (2013). Cyclic dinucleotides trigger ULK1 (ATG1) phosphorylation of STING to prevent sustained innate immune signaling. Cell 155, 688-698.

Kundu, M., Lindsten, T., Yang, C.Y., Wu, J., Zhao, F., Zhang, J., Selak, M.A., Ney, P.A., and Thompson, C.B. (2008). Ulk1 plays a critical role in the autophagic clearance of mitochondria and ribosomes during reticulocyte maturation. Blood 112, 14931502.

Lai, T., and Garriga, G. (2004). The conserved kinase UNC-51 acts with VAB-8 and UNC-14 to regulate axon outgrowth in C. elegans. Development 131, 5991-6000.

Lee, E.J., and Tournier, C. (2011). The requirement of uncoordinated 51-like kinase 1 (ULK1) and ULK2 in the regulation of autophagy. Autophagy 7, 689-695.

Levine, B., and Kroemer, G. (2008). Autophagy in the pathogenesis of disease. Cell 132, 27-42.

Li, T.Y., Sun, Y., Liang, Y., Liu, Q., Shi, Y., Zhang, C.S., Zhang, C., Song, L., Zhang, P., Zhang, X., et al. (2016). ULK1/2 Constitute a Bifurcate Node Controlling Glucose Metabolic Fluxes in Addition to Autophagy. Mol Cell 62, 359-370.

Liang, C.C., Wang, C., Peng, X., Gan, B., and Guan, J.L. (2010). Neural-specific deletion of FIP200 leads to cerebellar degeneration caused by increased neuronal death and axon degeneration. J Biol Chem 285, 3499-3509.

Liang, Q., Yang, P., Tian, E., Han, J., and Zhang, H. (2012). The C. elegans ATG101 homolog EPG-9 directly interacts with EPG-1/Atg13 and is essential for autophagy. Autophagy 8, 1426-1433.

Lim, J., Lachenmayer, M.L., Wu, S., Liu, W., Kundu, M., Wang, R., Komatsu, M., Oh, Y.J., Zhao, Y., and Yue, Z. (2015). Proteotoxic stress induces phosphorylation of p62/SQSTM1 by ULK1 to regulate selective autophagic clearance of protein aggregates. PLoS Genet 11, e1004987.

Lin, S.Y., Li, T.Y., Liu, Q., Zhang, C., Li, X., Chen, Y., Zhang, S.M., Lian, G., Liu, Q., Ruan, K., et al. (2012). GSK3-TIP60-ULK1 signaling pathway links growth factor deprivation to autophagy. Science 336, 477-481.

Lopez-Bendito, G., and Molnar, Z. (2003). Thalamocortical development: how are we going to get there? Nat Rev Neurosci 4, 276-289.

Mack, H.I., Zheng, B., Asara, J.M., and Thomas, S.M. (2012). AMPK-dependent phosphorylation of ULK1 regulates ATG9 localization. Autophagy 8, 1197-1214.

Maiuri, M.C., Zalckvar, E., Kimchi, A., and Kroemer, G. (2007). Self-eating and selfkilling: crosstalk between autophagy and apoptosis. Nat Rev Mol Cell Biol 8, 741-752.

Matsuura, A., Tsukada, M., Wada, Y., and Ohsumi, Y. (1997). Apg1p, a novel protein kinase required for the autophagic process in Saccharomyces cerevisiae. Gene 192, 245-250.

McIntire, S.L., Garriga, G., White, J., Jacobson, D., and Horvitz, H.R. (1992). Genes necessary for directed axonal elongation or fasciculation in C. elegans. Neuron 8 , 307-322. 
Melendez, A., Talloczy, Z., Seaman, M., Eskelinen, E.L., Hall, D.H., and Levine, B. (2003). Autophagy genes are essential for dauer development and life-span extension in C. elegans. Science 301, 1387-1391.

Mercado, C.P., and Kilic, F. (2010). Molecular mechanisms of SERT in platelets: regulation of plasma serotonin levels. Mol Interv 10, 231-241.

Miceli, S., Negwer, M., van Eijs, F., Kalkhoven, C., van Lierop, I., Homberg, J., and Schubert, D. (2013). High serotonin levels during brain development alter the structural input-output connectivity of neural networks in the rat somatosensory layer IV. Front Cell Neurosci 7, 88.

Miki, Y., Tanji, K., Mori, F., Utsumi, J., Sasaki, H., Kakita, A., Takahashi, H., and Wakabayashi, K. (2016). Alteration of Upstream Autophagy-Related Proteins (ULK1, ULK2, Beclin1, VPS34 and AMBRA1) in Lewy Body Disease. Brain Pathol 26, 359-370.

Miller, E.A., and Barlowe, C. (2010). Regulation of coat assembly--sorting things out at the ER. Curr Opin Cell Biol 22, 447-453.

Mizushima, N. (2010). The role of the Atg1/ULK1 complex in autophagy regulation. Curr Opin Cell Biol 22, 132-139.

Mizushima, N., Levine, B., Cuervo, A.M., and Klionsky, D.J. (2008). Autophagy fights disease through cellular self-digestion. Nature 451, 1069-1075.

Mizushima, N., Yoshimori, T., and Ohsumi, Y. (2011). The role of Atg proteins in autophagosome formation. Annu Rev Cell Dev Biol 27, 107-132.

Mochizuki, H., Toda, H., Ando, M., Kurusu, M., Tomoda, T., and Furukubo-Tokunaga, K. (2011). Unc-51/ATG1 controls axonal and dendritic development via kinesinmediated vesicle transport in the Drosophila brain. PLoS One 6, e19632.

Montgomery, T.R., Steinkellner, T., Sucic, S., Koban, F., Schuchner, S., Ogris, E., Sitte, H.H., and Freissmuth, M. (2014). Axonal targeting of the serotonin transporter in cultured rat dorsal raphe neurons is specified by SEC24C-dependent export from the endoplasmic reticulum. J Neurosci 34, 6344-6351.

Nazio, F., Strappazzon, F., Antonioli, M., Bielli, P., Cianfanelli, V., Bordi, M., Gretzmeier, C., Dengjel, J., Piacentini, M., Fimia, G.M., et al. (2013). mTOR inhibits autophagy by controlling ULK1 ubiquitylation, self-association and function through AMBRA1 and TRAF6. Nat Cell Biol 15, 406-416.

Nixon, R.A. (2013). The role of autophagy in neurodegenerative disease. Nat Med 19, 983-997.

Noda, N.N., and Fujioka, Y. (2015). Atg1 family kinases in autophagy initiation. Cell Mol Life Sci 72, 3083-3096.

Ogura, K., and Goshima, Y. (2006). The autophagy-related kinase UNC-51 and its binding partner UNC-14 regulate the subcellular localization of the Netrin receptor UNC-5 in Caenorhabditis elegans. Development 133, 3441-3450.

Ogura, K., Shirakawa, M., Barnes, T.M., Hekimi, S., and Ohshima, Y. (1997). The UNC14 protein required for axonal elongation and guidance in Caenorhabditis elegans interacts with the serine/threonine kinase UNC-51. Genes Dev 11, 1801-1811.

Ogura, K., Wicky, C., Magnenat, L., Tobler, H., Mori, I., Muller, F., and Ohshima, Y. (1994). Caenorhabditis elegans unc-51 gene required for axonal elongation encodes a novel serine/threonine kinase. Genes Dev 8, 2389-2400. 
Orsi, A., Razi, M., Dooley, H.C., Robinson, D., Weston, A.E., Collinson, L.M., and Tooze, S.A. (2012). Dynamic and transient interactions of Atg9 with autophagosomes, but not membrane integration, are required for autophagy. Mol Biol Cell 23, 1860-1873.

Papinski, D., and Kraft, C. (2016). Regulation of Autophagy By Signaling Through the Atg1/ULK1 Complex. J Mol Biol 428, 1725-1741.

Papinski, D., Schuschnig, M., Reiter, W., Wilhelm, L., Barnes, C.A., Maiolica, A., Hansmann, I., Pfaffenwimmer, T., Kijanska, M., Stoffel, I., et al. (2014). Early steps in autophagy depend on direct phosphorylation of Atg9 by the Atg1 kinase. Mol Cell 53, 471-483.

Park, J.M., Jung, C.H., Seo, M., Otto, N.M., Grunwald, D., Kim, K.H., Moriarity, B., Kim, Y.M., Starker, C., Nho, R.S., et al. (2016). The ULK1 complex mediates MTORC1 signaling to the autophagy initiation machinery via binding and phosphorylating ATG14. Autophagy 12, 547-564.

Petherick, K.J., Conway, O.J., Mpamhanga, C., Osborne, S.A., Kamal, A., Saxty, B., and Ganley, I.G. (2015). Pharmacological inhibition of ULK1 kinase blocks mammalian target of rapamycin (mTOR)-dependent autophagy. J Biol Chem 290, 11376-11383.

Presley, J.F., Cole, N.B., Schroer, T.A., Hirschberg, K., Zaal, K.J., and LippincottSchwartz, J. (1997). ER-to-Golgi transport visualized in living cells. Nature 389, 81-85.

Preston, A.M., Gurisik, E., Bartley, C., Laybutt, D.R., and Biden, T.J. (2009). Reduced endoplasmic reticulum (ER)-to-Golgi protein trafficking contributes to ER stress in lipotoxic mouse beta cells by promoting protein overload. Diabetologia 52, 2369-2373.

Reggiori, F., Tucker, K.A., Stromhaug, P.E., and Klionsky, D.J. (2004). The Atg1-Atg13 complex regulates Atg9 and Atg23 retrieval transport from the preautophagosomal structure. Dev Cell 6, 79-90.

Ro, S.H., Jung, C.H., Hahn, W.S., Xu, X., Kim, Y.M., Yun, Y.S., Park, J.M., Kim, K.H., Seo, M., Ha, T.Y., et al. (2013). Distinct functions of Ulk1 and Ulk2 in the regulation of lipid metabolism in adipocytes. Autophagy 9, 2103-2114.

Russell, R.C., Tian, Y., Yuan, H., Park, H.W., Chang, Y.Y., Kim, J., Kim, H., Neufeld, T.P., Dillin, A., and Guan, K.L. (2013). ULK1 induces autophagy by phosphorylating Beclin-1 and activating VPS34 lipid kinase. Nat Cell Biol 15, 741-750.

Saleiro, D., Mehrotra, S., Kroczynska, B., Beauchamp, E.M., Lisowski, P., MajchrzakKita, B., Bhagat, T.D., Stein, B.L., McMahon, B., Altman, J.K., et al. (2015). Central role of ULK1 in type I interferon signaling. Cell Rep 11, 605-617.

Schuck, S., Prinz, W.A., Thorn, K.S., Voss, C., and Walter, P. (2009). Membrane expansion alleviates endoplasmic reticulum stress independently of the unfolded protein response. J Cell Biol 187, 525-536.

Scott, R.C., Juhasz, G., and Neufeld, T.P. (2007). Direct induction of autophagy by Atg1 inhibits cell growth and induces apoptotic cell death. Curr Biol 17, 1-11.

Shin, S.H., Lee, E.J., Chun, J., Hyun, S., and Kang, S.S. (2015). ULK2 Ser 1027 Phosphorylation by PKA Regulates Its Nuclear Localization Occurring through Karyopherin Beta 2 Recognition of a PY-NLS Motif. PLoS One 10, e0127784. 
Sucic, S., El-Kasaby, A., Kudlacek, O., Sarker, S., Sitte, H.H., Marin, P., and Freissmuth, M. (2011). The serotonin transporter is an exclusive client of the coat protein complex II (COPII) component SEC24C. J Biol Chem 286, 16482-16490.

Suzuki, K., Akioka, M., Kondo-Kakuta, C., Yamamoto, H., and Ohsumi, Y. (2013). Fine mapping of autophagy-related proteins during autophagosome formation in Saccharomyces cerevisiae. J Cell Sci.

Sze, J.Y., Victor, M., Loer, C., Shi, Y., and Ruvkun, G. (2000). Food and metabolic signalling defects in a Caenorhabditis elegans serotonin-synthesis mutant. Nature 403, 560-564.

Tian, E., Wang, F., Han, J., and Zhang, H. (2009). epg-1 functions in autophagyregulated processes and may encode a highly divergent Atg13 homolog in $\mathrm{C}$. elegans. Autophagy 5, 608-615.

Toda, H., Mochizuki, H., Flores, R., 3rd, Josowitz, R., Krasieva, T.B., Lamorte, V.J., Suzuki, E., Gindhart, J.G., Furukubo-Tokunaga, K., and Tomoda, T. (2008). UNC-51/ATG1 kinase regulates axonal transport by mediating motor-cargo assembly. Genes Dev 22, 3292-3307.

Tomoda, T., Bhatt, R.S., Kuroyanagi, H., Shirasawa, T., and Hatten, M.E. (1999). A mouse serine/threonine kinase homologous to C. elegans UNC51 functions in parallel fiber formation of cerebellar granule neurons. Neuron 24, 833-846.

Tomoda, T., Kim, J.H., Zhan, C., and Hatten, M.E. (2004). Role of Unc51.1 and its binding partners in CNS axon outgrowth. Genes Dev 18, 541-558.

Tsukada, M., and Ohsumi, Y. (1993). Isolation and characterization of autophagydefective mutants of Saccharomyces cerevisiae. FEBS Lett 333, 169-174.

van Kleef, E.S., Gaspar, P., and Bonnin, A. (2012). Insights into the complex influence of 5-HT signaling on thalamocortical axonal system development. Eur J Neurosci $35,1563-1572$.

Walter, P., and Ron, D. (2011). The unfolded protein response: from stress pathway to homeostatic regulation. Science 334, 1081-1086.

Wang, C., Liang, C.C., Bian, Z.C., Zhu, Y., and Guan, J.L. (2013). FIP200 is required for maintenance and differentiation of postnatal neural stem cells. Nat Neurosci 16, $532-542$.

Watari-Goshima, N., Ogura, K., Wolf, F.W., Goshima, Y., and Garriga, G. (2007). C. elegans VAB-8 and UNC-73 regulate the SAX-3 receptor to direct cell and growth-cone migrations. Nat Neurosci 10, 169-176.

Watson, P., Townley, A.K., Koka, P., Palmer, K.J., and Stephens, D.J. (2006). Sec16 defines endoplasmic reticulum exit sites and is required for secretory cargo export in mammalian cells. Traffic 7, 1678-1687.

Witte, K., Schuh, A.L., Hegermann, J., Sarkeshik, A., Mayers, J.R., Schwarze, K., Yates, J.R., 3rd, Eimer, S., and Audhya, A. (2011). TFG-1 function in protein secretion and oncogenesis. Nat Cell Biol 13, 550-558.

Wong, P.M., Puente, C., Ganley, I.G., and Jiang, X. (2013). The ULK1 complex: sensing nutrient signals for autophagy activation. Autophagy 9, 124-137.

Wu, J., and Chen, Z.J. (2014). Innate immune sensing and signaling of cytosolic nucleic acids. Annu Rev Immunol 32, 461-488. 
Wu, J., Sun, L., Chen, X., Du, F., Shi, H., Chen, C., and Chen, Z.J. (2013). Cyclic GMPAMP is an endogenous second messenger in innate immune signaling by cytosolic DNA. Science 339, 826-830.

Wu, W., Tian, W., Hu, Z., Chen, G., Huang, L., Li, W., Zhang, X., Xue, P., Zhou, C., Liu, L., et al. (2014). ULK1 translocates to mitochondria and phosphorylates FUNDC1 to regulate mitophagy. EMBO Rep 15, 566-575.

$\mathrm{Xu}$, J., Fotouhi, M., and McPherson, P.S. (2015). Phosphorylation of the exchange factor DENND3 by ULK in response to starvation activates Rab12 and induces autophagy. EMBO Rep 16, 709-718.

Xu, P., Duong, D.M., and Peng, J. (2009). Systematical optimization of reverse-phase chromatography for shotgun proteomics. J Proteome Res 8, 3944-3950.

Yan, J., Kuroyanagi, H., Kuroiwa, A., Matsuda, Y., Tokumitsu, H., Tomoda, T., Shirasawa, T., and Muramatsu, M. (1998). Identification of mouse ULK1, a novel protein kinase structurally related to C. elegans UNC-51. Biochem Biophys Res Commun 246, 222-227.

Yan, J., Kuroyanagi, H., Tomemori, T., Okazaki, N., Asato, K., Matsuda, Y., Suzuki, Y., Ohshima, Y., Mitani, S., Masuho, Y., et al. (1999). Mouse ULK2, a novel member of the UNC-51-like protein kinases: unique features of functional domains. Oncogene 18, 5850-5859.

Young, A.R., Chan, E.Y., Hu, X.W., Kochl, R., Crawshaw, S.G., High, S., Hailey, D.W., Lippincott-Schwartz, J., and Tooze, S.A. (2006). Starvation and ULK1-dependent cycling of mammalian Atg9 between the TGN and endosomes. J Cell Sci 119, 3888-3900.

Zanetti, G., Pahuja, K.B., Studer, S., Shim, S., and Schekman, R. (2012). COPII and the regulation of protein sorting in mammals. Nat Cell Biol 14, 20-28.

Zhou, X., Babu, J.R., da Silva, S., Shu, Q., Graef, I.A., Oliver, T., Tomoda, T., Tani, T., Wooten, M.W., and Wang, F. (2007). Unc-51-like kinase 1/2-mediated endocytic processes regulate filopodia extension and branching of sensory axons. Proc Natl Acad Sci U S A 104, 5842-5847.

Ziu, E., Mercado, C.P., Li, Y., Singh, P., Ahmed, B.A., Freyaldenhoven, S., Lensing, S., Ware, J., and Kilic, F. (2012). Down-regulation of the serotonin transporter in hyperreactive platelets counteracts the pro-thrombotic effect of serotonin. J Mol Cell Cardiol 52, 1112-1121. 


\section{VITA}

Bo Wang was born in 1989 in Hunan province, China. He attained his Bachelor of Science degree in Biotechnology from Huazhong University of Science and Technology in 2011. In the same year, he was accepted into the Integrated Program in Biomedical Sciences at the University of Tennessee Health Science Center. In 2012, he joined the laboratory of Dr. Mondira Kundu in the Department of Pathology, St Jude Children's Research Hospital, and ever since he has been working on dissecting the physiological roles of ULK1 and ULK2 in the mouse brain. He is expected to graduate in December 2016 with Doctor of Philosophy, and then pursue a postdoc position in the United States. 